University of Tennessee Health Science Center UTHSC Digital Commons

\title{
Molecular Mechanism of Tetraspanin CD9 Mediated Cell Motility
}

Jayaprakash Kotha

University of Tennessee Health Science Center

Follow this and additional works at: https://dc.uthsc.edu/dissertations

Part of the Medical Cell Biology Commons, and the Medical Molecular Biology Commons

\section{Recommended Citation}

Kotha, Jayaprakash , "Molecular Mechanism of Tetraspanin CD9 Mediated Cell Motility" (2007). Theses and Dissertations (ETD). Paper 130. http://dx.doi.org/10.21007/etd.cghs.2007.0164.

This Dissertation is brought to you for free and open access by the College of Graduate Health Sciences at UTHSC Digital Commons. It has been accepted for inclusion in Theses and Dissertations (ETD) by an authorized administrator of UTHSC Digital Commons. For more information, please contact jwelch30@uthsc.edu. 


\title{
Molecular Mechanism of Tetraspanin CD9 Mediated Cell Motility
}

\begin{abstract}
$C D 9$, a member of the tetraspanin superfamily of proteins participates in the regulation of cell adhesive functions such as cell migration. The mechanisms underlying CD9 mediated cell migration are not known. In the current study, we investigated the molecular basis for the CD9 promoted cell migration. Our findings show that the phosphatidylinositol-3 kinase (PI-3K) inhibitors, wortamannin and LY294002 inhibited CD9 promoted cell motility in Chinese hamster ovary $(\mathrm{CHO})$ cells. In contrast, inhibitors targeting protein kinase $\mathrm{C}$ or mitogen-activated protein kinase had no effect on $\mathrm{CD} 9$ driven $\mathrm{CHO}$ cell motility. Furthermore, inhibition of PI-3K activity in $\mathrm{CHO}$ cells by dominant/negative PI-3K cDNA transfection abolished CD9 mediated pro-migratory effects. Consistent with these observations, CD9 expression in $\mathrm{CHO}$ cells and in the rat aortic smooth muscle (RASM) cells induced enhanced phosphorylation of PI-3K substrate, Akt. In $\mathrm{CHO}$ cells, CD9 expression also enhanced protein levels and tyrosine phosphorylation of the adaptor protein p130Cas. However, no significant changes in the CD9 enhanced migration were observed in $\mathrm{CHO}$ cells upon down regulation of p130Cas CD9, a member of the tetraspanin superfamily of proteins participates in the regulation of cell adhesive functions such as cell migration. The mechanisms underlying CD9 mediated cell migration are not known. In the current study, we investigated the molecular basis for the CD9 promoted cell migration. Our findings show that the phosphatidylinositol-3 kinase (PI-3K) inhibitors, wortamannin and LY294002 inhibited CD9 promoted cell motility in Chinese hamster ovary $(\mathrm{CHO})$ cells. In contrast, inhibitors targeting protein kinase $\mathrm{C}$ or mitogen-activated protein kinase had no effect on $\mathrm{CD} 9$ driven $\mathrm{CHO}$ cell motility. Furthermore, inhibition of $\mathrm{PI}-3 \mathrm{~K}$ activity in $\mathrm{CHO}$ cells by dominant/negative PI-3K cDNA transfection abolished CD9 mediated pro-migratory effects. Consistent with these observations, CD9 expression in $\mathrm{CHO}$ cells and in the rat aortic smooth muscle (RASM) cells induced enhanced phosphorylation of PI-3K substrate, Akt. In CHO cells, CD9 expression also enhanced protein levels and tyrosine phosphorylation of the adaptor protein p130Cas. However, no significant changes in the $\mathrm{CD} 9$ enhanced migration were observed in $\mathrm{CHO}$ cells upon down regulation of p130Cas by siRNA transfection suggesting that $\mathrm{p} 130 \mathrm{Cas}$ dependent pathways are not mandatory for CD9 mediated motility. To further understand the mechanisms by which CD9 regulates cell migration, we studied the relative contribution of the fibronectin (FN) receptor integrin, alpha-5-beta-1 in CD9 mediated cell motility. Our findings show that CD9 is in molecular complex with alpha-5-beta- 1 and that CD9 promoted migration can be completely blocked by an alpha-5-beta- 1 function blocking antibody. Further studies revealed that CD9 expression may stabilize the active conformer of the beta- 1 integrin. Taken togeather, our study demonstrates key molecular mechanisms governing CD9 mediated haptotactic cell motility to FN in $\mathrm{CHO}$ cells. Our findings indicate that CD9 in concert with integrin alpha-5-beta-1 requires activation of the PI-3K pathway leading to enhanced haptotactic cell migration on FN.
\end{abstract}

\section{Document Type}

Dissertation

Degree Name

Doctor of Philosophy (PhD)

Program

Molecular Sciences

Research Advisor

Lisa K. Jennings, Ph.D. 


\section{Keywords}

Tetraspanins, CD9, Integrins, alpha-5-beta-1, Extracellular Matrix, Fibronectin, Cell Migration, Haptotaxis, PI-3 Kinase, p130Cas, Signaling

\section{Subject Categories}

Medical Cell Biology | Medical Molecular Biology | Medical Sciences | Medicine and Health Sciences 
MOLECULAR MECHANISMS OF TETRASPANIN CD9

MEDIATED CELL MOTILITY

\author{
A Dissertation \\ Presented for \\ The Graduate Studies Council \\ The University of Tennessee \\ Health Science Center
}

\author{
In Partial Fulfillment \\ Of the Requirements for the Degree \\ Doctor of Philosophy \\ From The University of Tennessee
}

By

Jayaprakash Kotha

May 2007 
Copyright (C) 2007 by Jayaprakash Kotha All rights reserved 


\section{DEDICATION}

This thesis is dedicated to my family members

who have provided endless support and

encouragement throughout my

academic career. 


\section{ACKNOWLEDGEMENTS}

I would like to thank my major professor, Dr. Lisa K. Jennings for her wonderful support and guidance. I would also like to express my gratitude to my committee members, Dr. Xin Zhang, Dr. Gadiparthi Rao, Dr. John Cox and Dr. Ken Nishimoto for their valuable suggestions and guidance in my research studies.

I would like to thank my colleagues at the Vascular Biology Center of Excellence, Dr. Celia Longhurst, Dr. Svetozar Grgurevich, Dr. Al Cook, Dr. Yi Lu, Dr. Chunxiang Zhang, Henry Speich, Shila Cholera, Sarah Rowe, Melanie White, Mary Holmes, Mary Jacoski, Rajini Krishnan, Charlett Golden, Kishore Kodali, Whitney Appling, Shila Cholera, and Sarah Hill for their support.

I would also like to take this opportunity to offer special thanks to my friends Amol Gupte, Arun Gopal, Dennis Martin, Himanshu Bhattacharjee, Himangi Jayakar,

Jeetendra Eswaraka, Judith Ammons, Manish Gupta, Prabodh Sadana, Salar Khan, and Susan Price, for their support throughout my stay in Memphis. I would like to thank Shirley Hancock for carefully reviewing this manuscript for style and format.

Finally, I acknowledge American Heart Association (AHA) Southeast affiliate for supporting my Ph.D. studies in the form of predoctoral fellowship. 


\begin{abstract}
CD9, a member of the tetraspanin superfamily of cell surface proteins, is expressed on a wide variety of cells where it participates in the regulation of cell adhesive functions. Despite numerous reports of CD9 effects on cell migration, the molecular mechanisms governing CD9 promoted cell migration are as yet unclear. Earlier studies have shown that expression of human CD9 in Chinese hamster ovary (CHO) cells caused increased cell migration to the extracellular matrix $(\mathrm{ECM})$ protein fibronectin $(\mathrm{FN})$ when compared to Mock control cells. The goal of this study was to determine the molecular basis for CD9 mediated cell motility to FN.

Using CHO cells as a model system, and pharmacological inhibitors of crucial signaling pathways known to participate in the signaling events that regulate cell motility, we investigated the mechanism driving CD9 enhanced cell motility. Our findings show that the phosphatidylinositol-3 kinase (PI-3K) inhibitors, wortamannin and LY294002 inhibited CD9 effects on $\mathrm{CHO}$ cell motility. In contrast, inhibitors targeting protein kinase $\mathrm{C}(\mathrm{PKC})$ or mitogen-activated protein kinase (MAPK) had no effect on CD9 driven CHO cell motility.
\end{abstract}

To confirm the key role for PI-3K in CD9 mediated cell migration, dominant/negative PI-3K cDNA constructs were used to inhibit endogenous PI-3K activity in $\mathrm{CHO}$ cells. Motility assays performed on PI-3K dominant/negative transfected CD9-CHO cells confirmed that PI-3K was essential for CD9 promoted haptotactic cell motility on FN. Consistent with these observations, CD9 expression in $\mathrm{CHO}$ cells and in 
the rat aortic smooth muscle (RASM) cells induced enhanced phosphorylation of Akt, a downstream signaling event that reflects PI-3K activity.

Focal adhesion kinase (FAK) and the adaptor molecule p130Cas are well characterized participants in signaling pathways regulating cell motility. FAK acts to transmit signaling events from integrins in response to ECM. The activated FAK molecule provides a stage for the initiation of multiple signaling events including the PI3K/Akt pathway and the p130Cas pathway. The p130Cas adaptor protein participates in the p130Cas/Crk pathway, a regulatory mechanism governing directional cell migration in many cell types including $\mathrm{CHO}$ cells. For these reasons it was important to determine the activation states of FAK and p130Cas when CD9 was expressed. As the extent of tyrosine phosphorylation of both FAK and p130Cas is directly proportional to their activation states, the tyrosine phosphorylation states of these proteins were examined in CD9-CHO cells on adhesion to FN.

In comparison to Mock controls, an increase in p130Cas tyrosine phosphorylation was observed in CD9-CHO cells. Under parallel conditions, there were no significant changes in FAK tyrosine phosphorylation. Further examination revealed that p130Cas total protein levels were considerably increased in CD9 expressing cells compared to Mock transfectants. In spite of this observation, no significant changes in the pro migratory phenotype of CD9 expressing cells were seen on p130Cas siRNA specific knockdown. These data suggest that although p130Cas activation is a consequence of CD9 expression, p130Cas dependent pathways are not mandatory for CD9 mediated motility on FN. 
Integrins are cell surface molecules that act as receptors for ECM proteins. CD9 and other tetraspanins are known to participate in molecular complexes with integrins at the cell membrane. Integrin $\alpha 5 \beta 1$, the primary cell receptor for FN, plays a key role in cellular adhesive responses to FN. For these reasons, it is feasible that CD9 may influence the function of $\alpha 5 \beta 1$ via physical association at the cell membrane. Co-IP studies showed that CD9 was in complex with $\alpha 5 \beta 1$ at the cell membrane and CD9/ $\alpha 5 \beta 1$ association was detected in cells expressing CD9 both ectopically and constitutively. These data demonstrated that $\mathrm{CD} 9 / \alpha 5 \beta 1$ complexes were not an artifact formed as a result of exogenous CD9 expression.

In the current study a key role for $\alpha 5 \beta 1$ in CD9 mediated cell motility was demonstrated by the ability of function blocking monoclonal antibody $(\mathrm{mAb})$ against $\alpha 5 \beta 1$ to arrest cell motility in CD9-CHO cells. Since integrin $\alpha 5 \beta 1$ activation via change in conformation is required for cell migration on $\mathrm{FN}$, we examined whether CD9 expression affected $\alpha 5 \beta 1$ conformation state. In order to determine CD9 effects on the activation state of $\alpha 5 \beta 1$, binding of $\beta 1$ integrin conformation sensitive antibody (B44) in the presence of $\alpha 5 \beta 1$ activating peptide (RGDS) was measured in CD9-CHO vs. Mock cells. Our results showed that B44 antibody binding was significantly higher in CD9 expressing cells as compared with the control cells.

In summary, the study demonstrates key molecular mechanisms governing CD9 mediated haptotactic cell motility to FN in $\mathrm{CHO}$ cells. Our findings indicate that $\mathrm{CD} 9$ in concert with integrin $\alpha 5 \beta 1$ requires activation of the PI-3K pathway leading to enhanced haptotactic cell migration on FN. 


\section{TABLE OF CONTENTS}

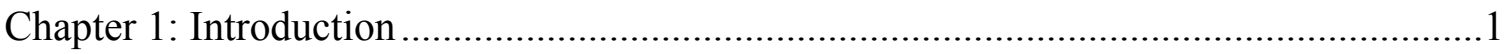

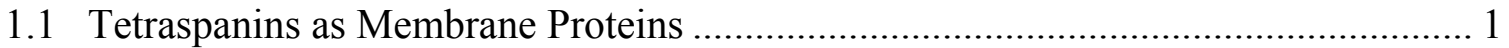

1.2 Common Structural Features of Tetraspanins...................................................... 3

1.3 Biological Significance of Tetraspanins ………................................................ 10

1.4 Genetic Evidence for the Biological Significance of Tetraspanins .......................... 13

1.5 History and Molecular Biochemistry of Tetraspanin CD9 ..................................... 17

1.6 Expression and Tissue Distribution of CD9 ……............................................. 20

1.7 Functional Significance of CD9 in Platelets …………........................................... 22

1.8 The Role of CD9 in Modulation of Adhesion Dependent Phenomenon .................. 24

1.9 CD9 Interactions with Cell Surface Molecules ................................................. 28

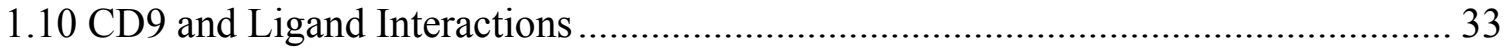

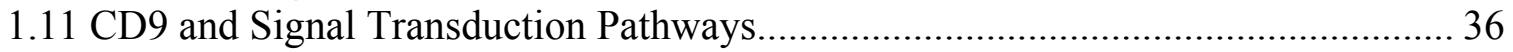

Chapter 2: Study Objectives ..................................................................................

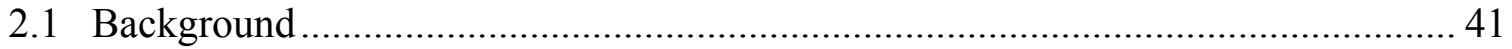

2.2 CD9 Mediated Signaling Pathways in Cell Motility ................................................ 43

2.3 Role of Integrins in CD9 Mediated Cell Motility ...................................................... 44

Chapter 3: Experimental Procedures .......................................................................47

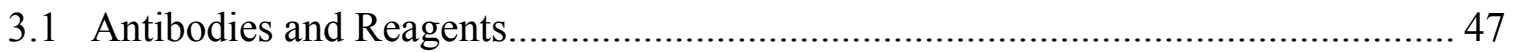

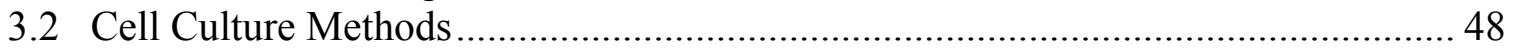

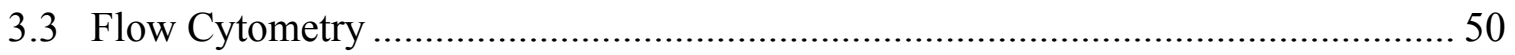

3.4 Immunoprecipitation and Western Blot Analysis of Platelet Antigens .................... 51

3.5 Generation and Purification of CD9-Adenovirus …………………………............ 55

3.6 Immunoprecipitation and Western Blot Analysis of Cultured Cells ........................ 57

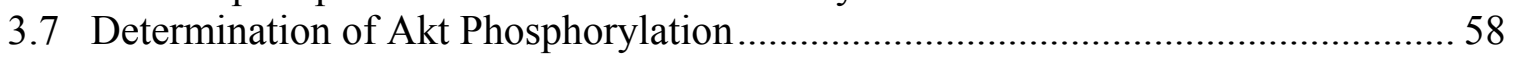

3.8 Determination of Protein Tyrosine Phosphorylation ................................................ 61

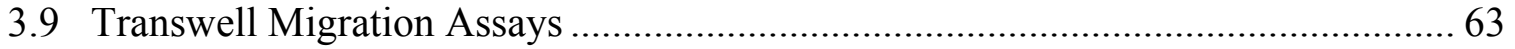

3.10 Transient Transfection with Dominant/Negative PI-3K........................................... 65

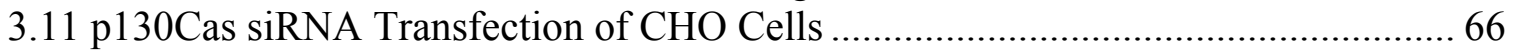

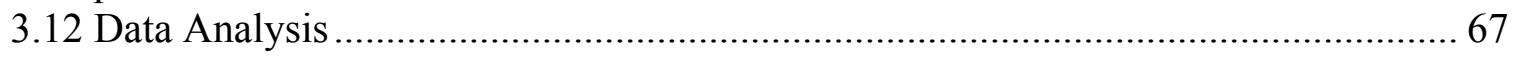

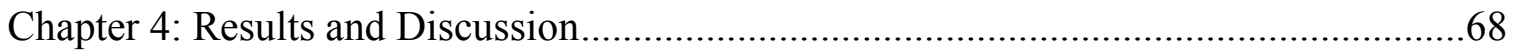

4.1 Signal Transduction Mechanisms of CD9 Mediated Cell Migration ........................ 68

4.2 PI-3K in CD9 Mediated Cell Motility ............................................................. 78

4.3 Adenoviral Vectors for Transient Expression of CD9............................................ 84

4.4 Effects of Transient Expression of CD9 on Akt Phosphorylation............................. 93

4.5 The Role of p130Cas in CD9 Mediated Cell Motility ……....................................... 96 


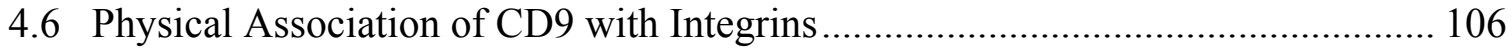

4.7 The Role of Integrins in CD9 Mediated Cell Motility....................................... 117

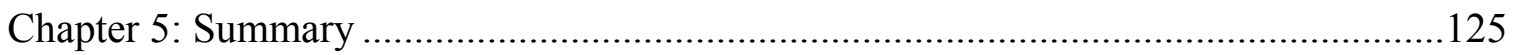

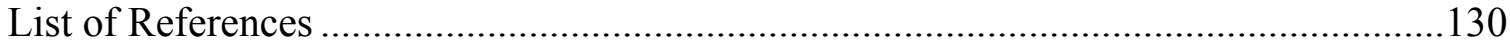

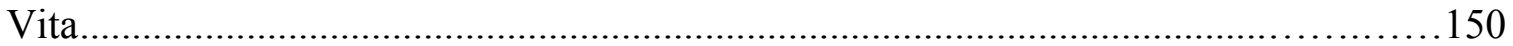




\section{LIST OF TABLES}

Table 1.1 Biological Functions of Membrane Proteins ................................................ 2

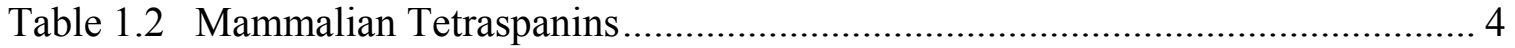

Table 1.3 Major Phenotypic Differences in Tetraspanin Knockout (KO) Mice............. 15

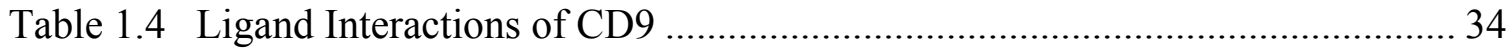

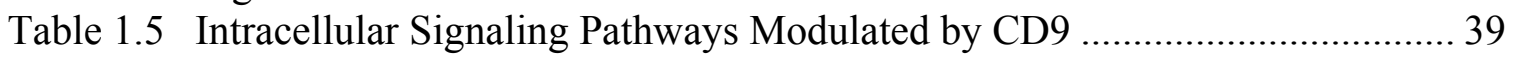

Table 3.1 Composition of Brij-98 Lysis Buffer ........................................................... 53

Table 3.2 Antibodies Used for the Characterization of CD9-GPIIb/IIIa Complex ........ 54

Table 3.3 Antibodies Used for the Detection of CD9- $\alpha 5 \beta 1$ Complex .......................... 59

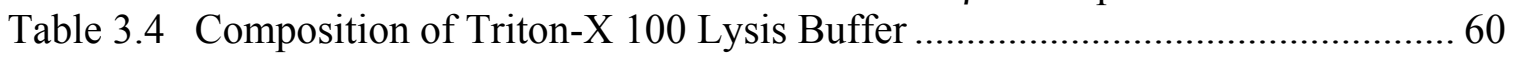

Table 3.5 Antibodies Used for the Western Blot Analysis of CHO Cell Lysates .......... 62

Table 4.1 nhibitors Used in CHO Cell Haptotactic Motility Experiments .................... 71

Table 4.2 Comparison of CD9 and Integrin Expression in RASM Cells ...................... 91 


\section{LIST OF FIGURES}

Figure 1.1 Schematic Representation of Tetraspanin Structure .................................. 7

Figure 1.2 Primary Amino Acid Sequence of Human CD9 ...................................... 19

Figure 1.3 Molecular Interactions of CD9 with Membrane Proteins........................... 29

Figure 2.1 Hypothesized Mechanisms of CD9 Mediated Cell Migration...................... 46

Figure 4.1 Effects of PKC Inhibitor GÖ 6976 on Cell Motility...................................... 73

Figure 4.2 Effects of MAPK Inhibitor PD98059 on Cell Motility ............................. 75

Figure 4.3 Effects of PI-3K Inhibitor Wortmannin on Cell Motility ........................... 77

Figure 4.4 Effects of PI-3K Inhibitor LY294002 on Cell Motility .............................. 79

Figure 4.5 Western Blot Analysis after Dominant/Negative PI-3K Transfection ......... 81

Figure 4.6 Effects of PI-3K Dominant/Negative $(\Delta \mathrm{p} 85)$ Transfection on Cell Motility 83

Figure 4.7 Effects of Stable Expression of CD9 on Akt Phosphorylation..................... 85

Figure 4.8 Quantification of Akt Phosphorylation in Mock and A6 Cells.................... 86

Figure 4.9 Western Blot Analysis for CD9 Expression after Adenoviral Transduction 88

Figure 4.10 FCM Analysis of CD9 Expression in RASM Cells................................. 89

Figure 4.11 FCM Analysis of Integrin Expression in RASM Cells.............................. 92

Figure 4.12 Effects of Transient Expression of CD9 on Akt Phosphorylation................ 94

Figure 4.13 Quantification of Akt Phosphorylation in RASM Cells ............................ 95

Figure 4.14 Analysis of Protein Tyrosine Phosphorylation in Mock and A6 Cells........ 97

Figure 4.15 Western Blot Analysis of p130Cas Expression in Mock and A6 Cells....... 99

Figure 4.16 Quantitative Comparison p130Cas Expression in Mock and A6 Cells ...... 100

Figure 4.17 Western Blot Analysis of p130Cas Tyrosine Phosphorylation.................. 102

Figure 4.18 Western Blot Analysis after p130Cas siRNA Transfection........................ 104

Figure 4.19 Motility of Mock and A6 cells after p130Cas siRNA Transfection ........... 105

Figure 4.20 Association of CD9-GPIIb/IIIa in Platelets .............................................. 108

Figure 4.21 Association of CD9 and Integrin $\beta 1$ in HT1080 Cells ............................. 110

Figure 4.22 Association of CD9 with Integrin $\beta 1$ in RASM Cells ............................... 111

Figure 4.23 Association of CD9 with Integrin $\alpha 5$ in RASM Cells .............................. 113

Figure 4.24 FCM Analysis of CD9 and $\alpha 5 \beta 1$ Expression in MEF............................. 115

Figure 4.25 Association of CD9 with Integrin $\alpha 5$ in MEF ......................................... 116

Figure 4.26 Effects of Serum on Mock and A6 Cell Migration ................................... 119

Figure 4.27 Effects of $\alpha 5 \beta 1$ Blocking Antibody (PB1) on A6 Cell Migration ............. 121

Figure 4.28 FCM Analysis of B44 Antibody Binding to Mock and A6 Cells.............. 124

Figure 5.1 Summary of Events Leading to CD9 Mediated Cell Motility .................... 129 


\section{LIST OF ABBREVIATIONS}

ACD Acid citrate dextrose

ALL Acute lymphocytic leukemia ATCC American Type Culture Collection

BSA Bovine serum albumin

CD Cluster differentiation

CEA Carcinoembyonic antigen

$\mathrm{CHO}$ Chinese hamster ovary

CLSM Confocal laser scanning microscopy

DAR Donkey anti-rabbit DC Dendritic cells

DMEM Dulbecco's modified eagle's medium

DMSO Dimethyl sulfoxide

EC-1 Extracellular loop-1

EC-2 Extracellular loop-2

ECM Extracellular matrix

EDTA Ethylene diamine tetra-acetic acid EGF . Epidermal growth factor ERK. Extracellular signal-regulated kinase ES Embryonic stem FAK. Focal adhesion kinase FBS .Fetal bovine serum 
FCM Flow cytometry

FG Fibrinogen

FITC Fluorescein Isothiocyanate

FN Fibronectin

GAM Goat anti-mouse

GAR Goat anti-rabbit

GFP Green fluorescent protein HEK Human embryonic kidney HRP Horseradish peroxidase $\operatorname{Ig}$ Immunoglobulin

IL-16 Interleukin-16

IP Immunoprecipitation JNK c-Jun $\mathrm{NH}_{2}$ terminal kinase

$\mathrm{KO}$ Knockout

$\mathrm{LN}$ .Laminin $\mathrm{mAb}$ Monoclonal antibody MAG Mouse anti-goat MAPK Mitogen-activated protein kinase MEF Mouse embryonic fibroblasts MFI Mean fluorescence intensity MHC Major histocompatibiliy complex MMP Matrix metalloprotenase MOI Multiplicity of infection 
mRNA Messenger RNA

MW Molecular weight

PAGE Polyacrylamide gel electrophoresis

PBS Phosphate buffered saline PI-3K Phosphatidylinositol-3 kinase

PI-4K Phosphatidylinositol-4 kinase

PKC Protein kinase $\mathrm{C}$

PSG Pregnancy specific glycoprotein

RASM Rat aortic smooth muscle

RPMI Roswell part memorial institute

SAM Sheep anti-mouse SAPK Stress-activated protein kinase siRNA Small interference RNA

TALLA T-cell lymphoblastic leukemia associated TGF- $\alpha$ Transforming growth factor- $\alpha$

TM Transmembrane

TM4SF Transmembrane 4 superfamily VSMC Vascular smooth muscle cells WASP Wiskott-Aldrich syndrome protein 


\section{Chapter 1: Introduction}

\subsection{Tetraspanins as Membrane Proteins}

Membrane proteins represent $25-30 \%$ of total proteins encoded by the human genome. Integral membrane proteins constitute a subclass of membrane proteins that are characterized by the presence of one or more hydrophobic regions in their primary amino acid sequence (1). The hydrophobic regions of integral membrane proteins extend through the phospholipid bilayer and constitute their transmembrane (TM) domains (2). Integral membrane proteins with TM domains are known as transmembrane proteins (3). In addition to serving as major structural components of membrane, transmembrane proteins mediate important biological functions. Table 1.1 summarizes the important biological functions mediated by transmembrane proteins. Based on the number of TM domains present in the primary amino acid sequence, transmembrane proteins are further classified in to different superfamilies. For example, transmembrane proteins with seven membrane spanning domains are known as "seven transmembrane proteins".

Transmembrane proteins with four membrane spanning regions are classified under transmembrane 4 superfamily (TM4SF). TM4SF proteins are also known as tetraspanins or tetraspans. Although the presence of four transmembrane domains is a requirement for a protein to be a tetraspanin, additional features such as the presence of unique motifs in the second extracellular loop is an essential requirement for it to be classified as a true tetraspanin. 
Table 1.1 Biological Functions of Membrane Proteins

\begin{tabular}{cc} 
Function & Examples \\
\hline Membrane Transport & Ion channels, Glucose and Amino Acid \\
Extracellular Matrix Recognition & Transporters \\
Cell-cell Interactions & Integrins \\
Soluble Ligand Recognition & Intercellular Adhesion Molecules (ICAMs) \\
Signal Transduction & Growth Factor Receptors \\
\end{tabular}


Tetraspanins are expressed in all eukaryotic organisms. Homologs of tetraspanins have even been reported in the genome of invertebrates such as Drosophila melanogaster (4), Caenorhabditis elegans (5), and Schistosoma mansoni (6) suggesting that tetraspanins are evolutionarily a highly conserved class of proteins. Genome analysis identified that there are about 37 tetraspanin homologs in Drosophila melanogaster (4) and about 20 tetraspanins in Caenorhabditis elegans (5).

A tetraspanin-like protein has been reported from the eukaryotic unicellular organism Magnaporthe grisea (7). However, so far, there is no evidence to show that tetraspanins are expressed in prokaryotic organisms. Analysis of tetraspanins from different organisms shows a high degree of conservation in their primary amino acid sequence.

Mammalian tetraspanins are a widely expressed class of proteins and about 33 members have been reported (8). With the exception of mature red blood cells (RBC) that do not express tetraspanins most mammalian cells express one or more TM4SF proteins (9). TM4SF members are often recognized by their alternative names. Table $\mathbf{1 . 2}$ shows the list of mammalian tetraspanins identified thus far and their alternative names.

\subsection{Common Structural Features of Tetraspanins}

Early evidence for the structural organization of the tetraspanins came from hydropathic analysis studies of tetraspanin primary amino acid sequence. On average, there are about 250 amino acids in TM4SF members with molecular weight ranging from 22-32 $\mathrm{kDa}$. The predicted structure of tetraspanin is comprised of four transmembrane domains (TM1-TM4) and two extracellular domains where the $\mathrm{N}$ and $\mathrm{C}$-termini are 
Table 1.2 Mammalian Tetraspanins

\begin{tabular}{|c|c|c|}
\hline Serial No & Primary Name & Alternative Names \\
\hline 1 & Net-1, TM4-C & Tetraspanin-1 \\
\hline 2 & TSAPN-2 & Tetraspanin-2 \\
\hline 3 & TSPAN-3 & Tetraspanin-3, OAP-1, TM4SF-8 \\
\hline 4 & Tspan-4 & Tetraspanin-4, NAG-2, TM4SF-7 \\
\hline 5 & Net-4 & Tetraspanin-5 \\
\hline 6 & TM4SF-6 & Tetraspanin-6, Tspan-6, T245 \\
\hline 7 & TALLA-1 & Tetraspanin-7,CD231,TM4SF-2, A15 \\
\hline 8 & TM4SF-3 & Tetraspanin-8, CO-029 \\
\hline 9 & Net-5 & Tetraspanin-9 \\
\hline 10 & Occulospanin & Tetraspanin-10 \\
\hline 11 & XP_497334 & Tetraspanin-11 \\
\hline 12 & NET-2 & Tetraspanin-12 \\
\hline 13 & Net-6 & Tetraspanin-13 \\
\hline 14 & DC-TM4F2 & Tetraspanin-14 \\
\hline 15 & Net-7 & Tetraspanin-15 \\
\hline 16 & TM4-B & Tetraspanin-16,TM4SF-16 \\
\hline 17 & SB 134 & Tetraspanin-17 \\
\hline
\end{tabular}


Table 1.2 (Continued)

\begin{tabular}{ccc}
\hline Serial No & Primary Name & Alternative Names \\
\hline 18 & BAB-55318 & Tetraspanin-18 \\
19 & XP_08648 & Tetraspanin-19 \\
20 & Uroplakin 1b & Tetraspanin-20 \\
21 & Uroplakin 1a & Tetraspanin-21, UP1 \\
22 & Peripherin & Tetraspanin-22, RDS \\
23 & ROM & Tetraspanin-23 \\
24 & CD151 & Tetraspanin-24, PETA-3 \\
25 & CD53 & Tetraspanin-25 \\
26 & CD37 & Tetraspanin-26 \\
27 & CD82 & Tetraspanin-27, KAI-1 \\
28 & CD81 & Tetraspanin-28, TAPA-1 \\
29 & CD9 & Tetraspanin-29, p24, MRP-1, DRAP-27 \\
30 & CD63 & Tetraspanin-30, LAMP-3 \\
31 & SAS & Tetraspanin-31 \\
32 & TSSC6 & \\
\hline
\end{tabular}

Note: * Mouse tetraspanin that to date lack a human homolog. 
located intracellularly. A short intracellular chain links the $3^{\text {rd }}$ and $4^{\text {th }}$ transmembrane domains. The first extracellular domain (EC1) is relatively short with about 20-28 amino acids when compared to the second extracellular domain (EC2) that contains about 76131 amino acids.

One of the unique features of the tetraspanins is the presence of 4-8 cysteine residues in the EC2 domain. Two of the conserved cysteines in EC2 exist in a "CCG" amino acid motif that is considered to be the signature sequence of TM4SF (Figure 1.1). The cysteine residues of EC2 region including those in the CCG motif participate in intrachain disulfide bonds. In addition to this feature, TM4SF proteins often contain polar residues such as glutamate and glutamine in their TM3 and TM4 domains (Figure 1.1).

Thus the overall three dimensional conformation of the EC2 domain is not only determined by the number of cysteine residues but also by the permissible disulfide linkages among these cysteine residues. Because of the unique conformation of the EC2 domain, several monoclonal antibodies (mAbs) can bind tetraspanins with high specificity. Furthermore, most of the functions mediated by tetraspanins are attributable to this region. Figure 1.1 depicts the schematic drawing of a typical tetraspanin protein. Mammalian cells express several other membrane proteins that contain four transmembrane proteins. For example, proteins such as sarcospan (10), CD20 (11), like tetraspanins, contain four transmembrane domains. However, due to the absence of unique structural motifs seen in tetraspanins, these proteins are not classified under TM4SF.

Due to the difficulties inherent to purification, stabilization and crystallization of membrane proteins, structural information of full-length tetraspanin proteins is not 


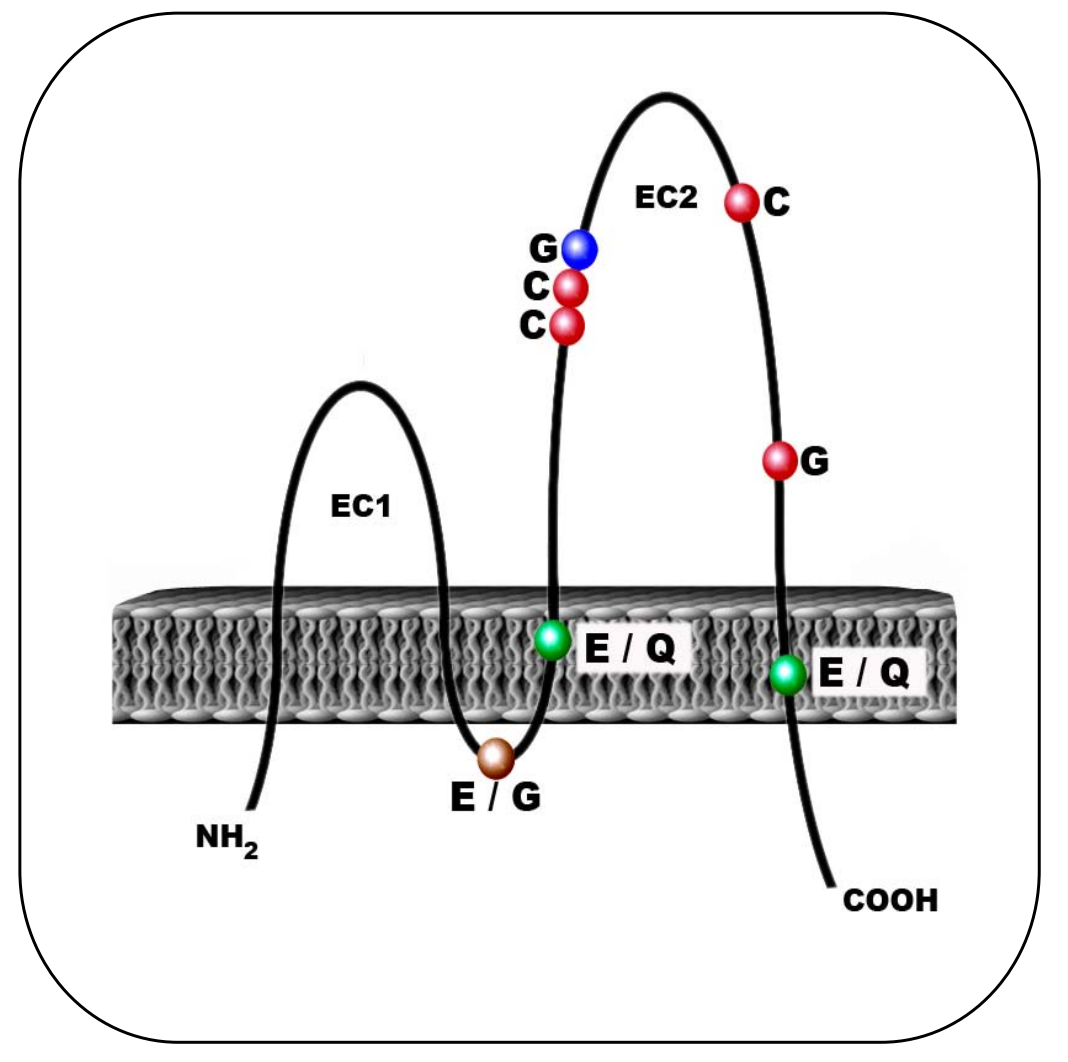

Figure 1.1 Schematic Representation of Tetraspanin Structure

The colored circles represent the highly conserved amino acids of the EC2, $3^{\text {rd }}$ and $4^{\text {th }}$ transmembrane domains and the intracellular loop. EC1: Extracellular loop-1, EC2: Extracellular loop-2; E: Glutamic acid, C: Cysteine, Q: Glutamine, G: Glycine. 
available. However, the crystal structure of tetraspanin CD81 EC2 domain has been solved $(12,13)$. The EC2 domain of CD81 was shown to contain five $\alpha$-helices arranged in a "stalk" and "head" subdomain pattern (12). Comparative structural analysis of other tetraspanins indicates that structural features in the CD81 EC2 are common to all tetraspanins. The unique domain structure of tetraspanins may facilitate homo/heterodimerization of tetraspanins.

The $\mathrm{N}$ and $\mathrm{C}$ terminal cytoplasmic domains of tetraspanins contain very few amino acids. The c-terminal cytoplasmic tails of a few tetraspanins have been shown to possess a conserved $\mathrm{YXX} \phi(\mathrm{X}$ : any amino acid; $\phi$ : hydrophobic amino acid) motif. The YXX $\phi$ motif of tetraspanins CD151, CD63, and CD82 represents a tyrosine based internalization motif that is required for endosomal/lysosomal recycling pathway (14, $15)$.

Transmembrane domains of tetraspanins are deemed essential for proper maturation, membrane targeting, and for maintaining intermolecular interactions $(16,17)$. Structurally, transmembrane domains of tetraspanins exhibit some distinctive features. For example, transmembrane domains TM1, TM2, possess a heptad repeat sequence with glycine as the first amino acid that is thought to be important for intramolecular interactions. TM3 domain, in addition to containing the heptad repeat, also contains highly conserved polar amino acid residues glutamate, glutamine in its sequence. TM4 domain lacks the heptad repeat sequence but contains the conserved polar amino acids glutamate and glutamine (16) (Figure 1.1).

Perhaps the most significant consequence of the tetraspanin structure is their ability to organize membrane proteins into distinct microdomains within membranes. 
Depending on the structural configuration of the EC2 domains, tetraspanins can associate with a wide variety of membrane proteins. Tetraspanin containing membrane protein complexes have been termed as tetraspanin enriched microdomains or TEMs (18). Within the TEMs, a few tetraspanins such as CD151 and CD81 are known to have a highly stoichiometric and avid physical interaction with other membrane proteins $(3,19)$. These interactions mediated by CD151 and CD81 are retained even after harsh detergent (1\% Triton-X100) treatment and reported to be due to direct interactions as demonstrated by chemical cross linking experiments (20). Other tetraspanin members reported to have direct interactions include the Uroplakins (21).

The direct interactions of CD151 and CD81 with other membrane proteins are thought to be mediated by their EC2 domains and are characterized by resistance to highly hydrophobic detergents such as Triton-X100. The dimerization motifs in the EC2 regions of few tetraspanins such as CD81 may also aid in the formation of homo/hetero dimeric complexes within the TEM. Most tetraspanins cannot maintain direct interactions described above and are thought to enter primary tetraspanin complexes by secondary associations. These secondary associations are easily disrupted by stringent detergents such as Triton-X100 but resist separation by mild detergents such as CHAPS and Brij-98 (22). Secondary tetraspanin interactions are non stoichiometric and factors such as palmitoylation of tetraspanins were shown to significantly influence the nature of these interactions (23). 


\subsection{Biological Significance of Tetraspanins}

Although tetraspanins are widely studied, little knowledge has been attained in understanding the true biological significance of these proteins. Tetraspanins are known to form non-covalent associations with a wide variety of cell surface proteins. The complexity of tetraspanin interactions with other membrane proteins together with their broad tissue distribution makes tetraspanins a difficult family of proteins to understand in terms of their biological function. Tetraspanins, in addition to contributing to the structural framework of membranes, are also involved in several important biological processes such as embryonic development, infection by pathogens, and tumor cell metastasis.

The integrin family of adhesion molecules was the first group of membrane proteins detected in complex with tetraspanins. Structurally, integrins are heterodimeric molecules comprised of $\alpha$ and $\beta$ subunits (24-26). So far, about $18 \alpha$ and $8 \beta$ subunits of integrins have been characterized. Via the combination of $\alpha$ and $\beta$ subunits, 24 integrins are expressed in the mammalian cells. Tetraspanins have been detected in complex with integrins containing $\beta 1, \beta 3$, and $\beta 4$ subunits. Tetraspanin associations, with $\beta 1$ integrins in particular, are thought to modulate integrin mediated cell adhesive phenotypes $(19,27$, 28). Often more than one type of tetraspanin is present in the integrin-tetraspanin complexes and integrin-tetraspanin complexes in the cell membrane are thought to form a network known as the "tetraspanin web" (9).

Other cell surface molecules that can exist in complex with tetraspanins include the Ig superfamily of proteins, growth factors, and immune cell markers such as cluster differentiation $(\mathrm{CD})$ and major histocompatibility $(\mathrm{MHC})$ proteins. More specifically, 
CD81 and CD9 associate with Ig superfamily of protein EWI-2 (29). Evidence also suggests that tetraspanins may actually link members of the Ig superfamily with integrins thus modulating their functions (30). Additionally, CD81 was reported to associate with the helper T-lymphocytes marker CD4 (31) and CD9 was shown to associate with IgM (32).

So far few growth factors and their receptors have been detected in complex with tetraspanins. CD9 was shown to associate with and modulate the activity of membrane bound form of heparin binding epidermal growth factor-like growth factor (Pro-HBEGF) and modulate Pro-HBEGF activity $(33,34)$. Recently, CD9 and CD81 were isolated from ganglioside enriched microdomains along with the fibroblast growth factor receptor (35). Functional implications of tetraspanin-growth factor receptor interactions are still under investigation.

Tetraspanins have been shown to participate in integrin-associated cell signaling pathways. A specific link between $\alpha 3 \beta 1$, the tetraspanin CD151, and PI 4-kinase was observed where the PI 4-kinase activity was shown to be associated with CD151 immunoprecipitates $(36,37)$. This report was the first demonstration of a direct association between a tetraspanin member and an intracellular signaling molecule that regulates the cytoskeleton. Subsequently, other tetraspanins such as CD9, CD63, CD81 and TALLA-1 have also been reported to associate with PI 4-kinase (20).

Tetraspanins have also been shown to influence the association of $\alpha 3$ integrin containing complexes with specific isoforms of PKC. The formation of integrintetraspanin-PKC complexes was thought to influence localization of additional signaling complexes that regulate cytoskeletal organization (38). A recent study of TM4SF 
member, CD82, in the prostate cancer cell line DU145, suggested that modulation of p130Cas protein levels and its activation represents a mechanism by which TM4SF members influence cell motility and cancer metastasis (39).

Several studies have shown evidence for the role of tetraspanins in the regulatory processes of pathogen mediated infections. Tetraspanin CD81 was reported to act as a receptor for hepatitis C virus (Hep-C) (40). More specifically, the regions of CD81 that are involved in Hep-C binding were mapped to the EC2 domain (amino acids: 164-201) where anti- CD81-EC2 mAbs also blocked Hep-C- CD81 binding. Although CD81 was the first receptor identified for Hep-C, several recent studies have shown that CD81 alone is not sufficient for Hep-C infection $(41,42)$. Recently, CD81 was also shown to play a role in the pathogenesis of malaria (43). Using hepatocytes from CD81 knockout mice and blocking antibodies against CD81, Silvie et al, 2003 (43) have shown that CD81 is required for the entry of Plasmodium sporozoites into hepatocytes, an essential step for completion of Plasmodium life cycle.

Other tetraspanins that have been shown to take part in the etiology of infections include the tetraspanins CD82, CD63 and CD9. Antibodies to CD82 inhibited human Tlymphotropic virus-1 (HTLV-1) induced syncitia of cultured cells $(44,45)$. Similarly, antibodies to CD63 inhibited human immuno deficiency virus type-1 (HIV-1) infection in cultured cells (46). Antibodies to CD9 and CD81 modulated HIV-1 induced syncitia formation of cultured cells (47).

In addition to participating in pathogenesis, tetraspanins also modulate the host's immune response to infections. Several tetraspanins associate with proteins that are directly or indirectly involved in modulating immune response. For example, tetraspanins 
CD9, CD81, CD63 and CD53 were all shown to associate with the class II major histocompatibility complex proteins that are important in antigen presentation to the immune cells (48). In T helper lymphocytes, CD81 and CD82 associate with CD4 (31), the primary receptor for HIV-1 viral entry. Additionally, tetraspanins have been implicated in the regulation leukocyte activation, migration, and response to antigens (49).

The role of tetraspanins in the regulation of tumor cell metastasis has been intensely investigated. Expression of various tetraspanins appears to significantly vary during the natural course of tumor growth and early reports have even proposed several tetraspanins as useful markers of tumor cells. For example TALLA-1 has been reported as a marker for T-lymphoid leukemias (50) while CD9 was recognized as a marker for B cell acute lymphoblastic leukemias (51). More recent studies have questioned the usefulness of tetraspanins such as CD9 as useful tumor markers, as some tumor cell types do not have the predicted tetraspanin expression profile (52). Although tetraspanins such as CD82 appear to be in general suppressors of tumor cells metastasis, a consensus has not been reached with regard to the role of many other tetraspanins in tumorogenesis and metastasis (53).

\subsection{Genetic Evidence for the Biological Significance of Tetraspanins}

Functionally relevant tetraspanins have been reported in invertebrates such as Magnaporthe grisea, Drosophila melanogaster, and Schistosoma hematobium. In Magnaporthe grisea, a pathogenic fungus that infects rice leaf, PLS1 gene has been shown to encode a tetraspanin-like protein. Inactivation of PLS1 was shown to impair the 
ability of the fungus to penetrate rice leaf cells suggesting that PLS1 gene product is essential for parasite entry in to the host cells (7). Genetic analysis also showed that the late bloomer $(\mathrm{lbl})$ gene in Drosophila melanogaster encodes tetraspanin protein, where specific mutations of $l b l$ were shown to delay synapse formation at neuro-muscular junctions (54).

One of the early genetic evidences for the biological significance of mammalian tetraspanins came from the observation that a balanced translocation involving Xchromosome inactivated T-cell acute lymphoblastic leukemia associated antigen-1 (TALLA-1) causing a form of X-linked mental retardation (55). Although originally identified as a leukemic T-cell antigen, TALLA-1, a tetraspanin is abundantly expressed in the central nervous system of human (55) and mice (56) suggesting that TALLA-1 may be important for functional maturation of nervous tissue.

Gene knockout studies have become a common method of studying the functional role of proteins. By engineering the mouse embryonic stem (ES) cells, it is possible to generate a transgenic mouse lacking the protein $[$ knockout $(\mathrm{KO})]$ of interest in all tissues. The consequence of gene knockouts for five different tetraspanins, CD9, CD81, CD37, CD151, and Tssc 6 have been investigated. Table $\mathbf{1 . 3}$ summarizes the major phenotypic changes reported in the tetraspanin $\mathrm{KO}$ mice.

The first tetraspanin KO phenotype reported was for CD9. Three independent groups have reported that female mice deficient in CD9 have reduced rates of fertility due to failure of oocytes to fertilize with sperm (57-59). Aside from reduced fertility, CD9 -/mice were healthy without any noticeable abnormalities. Tetraspanin TSSC also known as tetraspanin-32 or PHEMX is highly expressed in the hematopoietic and lymphoid 
Table 1.3 Major Phenotypic Differences in Tetraspanin Knockout (KO) Mice

\begin{tabular}{cc}
\hline Tetraspanin KO & Phenotype \\
\hline CD9 & Reduced Fertility in Female Mice \\
CD151 & Renal Failure \\
Hemostasis Abnormality \\
Impaired Outside-in Signaling in Platelets \\
Reduced Fertility in Female Mice \\
Reduced Infectivity of Hepatocytes with Malarial Parasite \\
Reduced Expression of CD19 and Ca ${ }^{2+}$ Signaling in B Cells \\
RS66 \\
Reduced T dependent B cell Immune Response \\
Impaired Outside-in Signaling in Platelets
\end{tabular}


system (60). Despite high level of expression in these tissues, no significant abnormalities were observed in the development of hematopoietic and lymphoid system of TSSC -/mice (61). The only major phenotypic differences observed in TSSC6 -/- mice was defective GPIIb/IIIa mediated “outside-in" signaling in platelets (62) and abnormal in vitro proliferative response of T- cells to antigens (61).

Tetraspanin CD37, also known as tetraspanin-26, was originally reported as a marker for B lymphocyte tumor cells (63) and was subsequently detected on several cells of the immune system (64). However, CD37 -/- mice did not exhibit significant anomalies in lymphoid organ development (64). The only abnormality observed in the CD37-/- mice appears to be the anomalous humoral immune response of B cells to Tcell-dependent antigens (64).

CD81, also known as TAPA-1 has a wide tissue distribution. The phenotype of CD81-/- mice was originally reported by Shoham et al, 2003 where CD81 deletion downregulated CD19 expression on B cells (65). CD81 was shown to associate with CD19, a unique marker of B cells (66). Subsequently, Silvie et al, 2003 reported that Plamodium yoelii sporozoites failed to infect CD81 -/- hepatocytes and CD81 expression in hepatocytes is required for differentiation of parasite into exoerythrocytic forms (43). Recently, CD81 -/- female mice were shown to have reduced fertility, the phenotype characteristic of CD9 -/- female mice (67).

CD151 is widely expressed tetraspanin and the CD151 KO phenotypes have been characterized. The predominant abnormalities of CD151 KO are associated with platelet function. CD151 -/- mice had minor bleeding abnormalities with prolonged bleeding times and increased incidence of rebleeding (68). In accordance with the bleeding 
anomalies observed in the CD151 -/- mice, platelets derived from CD151 -/- mice were shown to have impaired agonist induced GPIIb/IIIa mediated "outside-in" signaling response and diminished spreading on fibrinogen (FG) (69). CD151 -/- mice were also prone to renal failure (70).

Results from the KO models clearly indicate that the tetraspanins are dispensable for normal intra uterine growth and development of mice. Although tetraspanins CD81, CD9, CD37 are expressed in different sub populations of B lymphocytes, complete formation of mature B lymphocytes does not appear to require a full complement of tetraspanins. Despite abundant expression of tetraspanins CD9 and CD151 on platelets, hemostasis abnormalities thus far appear to be limited to CD151 -/- mice. Lack of CD9 did not influence formation or renewal of platelets or marginal Zone B cells where it is abundantly expressed.

A feature common to $\mathrm{CD} 9$ and $\mathrm{CD} 81$ homozygous deletion is the failure of $\mathrm{KO}$ murine ova to fuse normally with sperm. Interestingly, CD9, CD81 double KO mice were shown to be completely infertile as opposed to the partial infertile phenotype observed with CD9, CD81 single gene deletion (67). These findings suggest that tetraspanins have overlapping functional roles and that absence of one tetraspanin may lead to possible functional compensation by another closely related member.

\subsection{History and Molecular Biochemistry of Tetraspanin CD9}

Human CD9 was first reported via its reactivity with the monoclonal antibody (mAb), BA-2, raised against leukemic cell line, NALM-6 (51). The BA-2 antibody immunoprecipititaed a protein with a molecular weight of $24 \mathrm{kDa}$ and was termed the 
p24 antigen. Immunofluorescence assays demonstrated that p24 is a cell surface protein present on several bone marrow precursor cells of the lymphopoietic lineage. Subsequently, several mAbs were generated that specifically recognized p24 antigen expressed on a variety of cells (71-74). p24 antigen was named as the cluster of differentiation 9 (CD9) at the First International Workshop on Leukocyte Differentiation Antigens in the year 1984 (75).

Using specific DNA probes, the gene encoding p24/CD9 was localized to the short arm of chromosome 12 (76). The primary structure of p24/CD9 was elucidated in 1991 where CD9 cDNA was isolated from a megakaryocytic library. The CD9 gene consists of 8 coding regions (exons) that span about $20 \mathrm{~Kb}$ region of genome. Transcription of the $\mathrm{CD} 9$ gene generates a $1.4 \mathrm{~Kb}$ mRNA that encodes a protein of 228 amino acids with an apparent molecular weight of to a $24 \mathrm{kDa}(77)$.

Prototypically, CD9 is comprised of four highly hydrophobic transmembrane (TM) regions designated TM1-4, two extracellular regions (EC1 and EC2) and $\mathrm{NH}_{2}$ and $\mathrm{COOH}$ terminal cytoplasmic tails. Topography studies of CD9 reveal that both the Nterminal and C-terminal regions are located in the cytosolic portions of the cell (77). The TM domains of CD9 are also highly conserved including Glu or Gln residues within the $3^{\text {rd }}$ and $4^{\text {th }}$ TM domains (Figure 1.1). There are a total of ten cysteine residues in the primary amino acid sequence of $\mathrm{CD} 9$ and the conserved CCG motif is localized to the EC2. Figure 1.2 shows the primary amino acid sequence of CD9 with mapping of various structural domains.

Most integral membrane proteins are post translationally modified by the addition of one or more oligosaccharide chains (78). Addition of oligosaccharide chains 


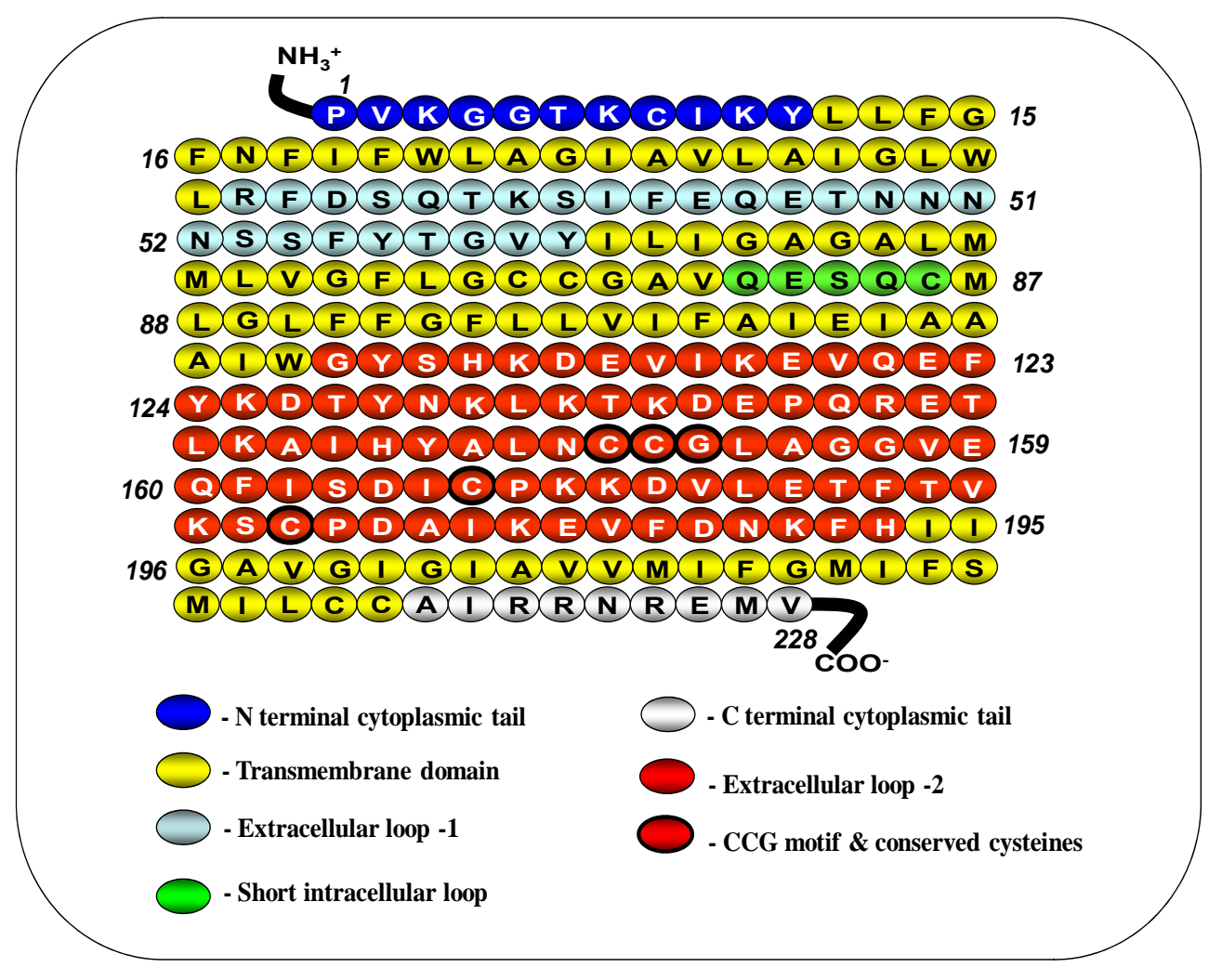

Figure 1.2 Primary Amino Acid Sequence of Human CD9 
(glycosylation) can occur either on the $\mathrm{OH}$ group of amino acids serine and threonine (Olinked glycosylation) or on the $\mathrm{NH} 2$ group of arginine (N-linked glycosylation). N-linked Glycosylation of tetraspanins has been hypothesized to regulate their functions (79). Primary amino acid sequence of CD9 reveals the presence of a classical N-linked glycosylation motif (NXaaS/T) in the EC1 region. Although CD9 lacks the NXaaS/T motif in the EC2 region, NXaaC sequence of the $\mathrm{EC} 2$ was thought to be glycosylated, however, the functional consequences of CD9 glycosylation are as yet unknown (80). Membrane proteins are also known to undergo post translational modification by the addition lipids (81). Such lipid modifications are thought to be important for targeting proteins to the membrane and for their assembly into multimeric complexes. Palmitoylation is a reversible lipid modification where fatty acids such as palmitic acid are added to cysteine residues of membrane proteins. Studies indicate that CD9 undergoes palmitoylation (23) and that palmitoylation may be critical for maintenance of tetraspanin-integrin interactions (82).

\subsection{Expression and Tissue Distribution of CD9}

Although originally identified in the cells derived from the hematopoietic lineage, CD9 is also constitutively expressed in several other tissues. Within the hematopoietic system, CD9 expression is most abundant in the megakaryocyte lineage where both

platelets and megakaryocytes express large quantities of CD9 (72, 83-85). CD9

expression has been detected on other hematopoietic derived cells including macrophages (86), eosinophils (87), basophils (88) and the lymphoid lineage (89). Although CD9 expression is not seen in the mature circulating/peripheral B and $\mathrm{T}$ lymphocytes (51), 
bone marrow lymphopoietic cells and mitogen activated lymphocytes however, express significant levels of CD9. In the nascent B cell sub population of spleen, CD9 is uniquely expressed on the marginal zone B (MZB) cells (89).

In the vascular system, CD9 is expressed on endothelial cells $(90,91)$ of the tunica intima and on vascular smooth muscle cells $(92,93)$ of the tunica media. The abnormal migration and proliferation vascular smooth muscle cells and endothelial cells are cellular events that occur during atherosclerosis and other major vascular disorders. Interestingly, CD9 has been implicated in the regulation of cell migration in both vascular smooth muscle cells and endothelial cells $(91,92)$.

$\mathrm{CD} 9$ expression is also found on ectodermal derived nervous tissue, where a mAb specific for human $\mathrm{CD} 9, \mathrm{~B} 2 \mathrm{C} 11$, induced morphological changes and promoted neurite outgrowth in the cultured neurons (94). CD9 is highly expressed in the developing mouse brain as well as in the adult mouse neuronal components such as the glial cells and the myelin sheaths (95). In the peripheral nervous system, CD9 is expressed on Schwann cells where anti-CD9 mAbs modulate in vitro Schwann cell migration (96).

In the reproductive system CD9 is expressed on the surface of oocytes. Oocytes derived from human (97), pig (98) and mouse (58) expressed CD9 where CD9 expression is essential for sperm-egg fusion. CD9 is also found on extra villuous trophoblasts (99) of the gravid uterus and the granulose cells of the ovary (100). CD9 is also expressed on all the living layers of epidermis (101) and on cultured fibroblasts and osteoclasts. Several tumor cells including those derived from hematopoietic lineage of cells also express CD9. Importantly, the extent of CD9 expression is altered on tumors and it has been 
proposed that there is a relationship between CD9 expression and metastatic potential in a number of human cancers.

\subsection{Functional Significance of CD9 in Platelets}

CD9 is abundantly expressed on the surface of platelets. One of the earliest and most striking observations related to platelet CD9 function is the ability of anti-CD9 mAbs to cause platelet activation and aggregation $(102,103)$. Most of the anti-CD9 mAbs tested induce platelet aggregation evev in the absence of exogenous fibrinogen. Furthermore, platelet aggregation induced by anti-CD9 mAbs requires the intact immunoglobulin antibody. Purified Fab fragments derived from anti-CD9 mAbs were unable to elicit platelet aggregation. Based on these findings, it has been hypothesized that anti-CD9 mAbs induce platelet aggregation response by cross linking the $\mathrm{Fc}$ receptors and CD9.

Platelets also express tetraspanins CD151 (69) and CD63 (104). In resting platelets, both CD9 and CD151 are located at the platelet membrane where activation of platelets does not change the surface expression of these tetraspanins. Conversely, in resting platelets CD63 is predominantly localized to the intracellular granules (105) and platelet activation and degranulation promotes CD63 translocation to the peripheral membrane resulting increased CD63 levels at the platelet surface (106).

Another interesting feature of platelet CD9 is its ability to form complexes with the platelet integrin GPIIb/IIIa ( $\alpha \mathrm{IIb} \beta 3$ ). GPIIb/IIIa, the fibrinogen receptor abundantly expressed on platelets, is essential for platelet aggregation where mutations in GPIIb/IIIa lead to an inherited bleeding disorder known as Glanzmann's thrombasthenia (107). The 
earliest reports of CD9/ GPIIb/IIIa association came from the studies of Slupsky et al, 1989 (108). Subsequently several groups have confirmed this observation $(84,109,110)$. Scanning electron microscopy studies have revealed that in activated platelets, CD9 and GPIIb/IIIa specifically localizes to membrane protrusions and platelet-platelet contact sites (84) suggesting that CD9 may participate in the regulation of adhesion dependent phenomena in platelets.

In addition GPIIb/IIIa, CD9 has been detected in complex with other major platelet membrane proteins such as the von Willebrand factor (vWF) receptor GPIb/V/IX (110). The functional consequences of CD9 with either GPIIb/IIIa or the GPIb/V/IX complexes are largely unknown, and it is speculated that CD9 may regulate the activation states of these glycoproteins. In addition to mediating protein-protein interactions in the platelets, CD9 was also shown to modulate intracellular signal transduction pathways within the platelets. Treatment of platelets with anti-CD9 mAbs alter intracellular calcium influx (111), phosphoinositide turnover, signaling through PKC, and platelet cytoskeletal reorganization (102).

Since CD9 is also expressed on the platelet precursor, the megakaryocytes, it is also speculated that CD9 may be required for megakaryocyte lineage development and platelet formation. Despite the effects of anti-CD9 mAbs on platelets, platelet function abnormalities have not have been reported in CD9 knockout mice. At least in the mouse model, even in the absence of $\mathrm{CD} 9$, platelets appear to be formed with normal structure and function. Although the reasons for the above observed discrepancies are unclear to date, it can be speculated that in the absence of $\operatorname{CD} 9$, other tetraspanins expressed on the platelets may have compensatory effects. 


\subsection{The Role of CD9 in Modulation of Adhesion Dependent Phenomenon}

Since the identification of CD9 as a cell surface protein, numerous studies have been performed to understand the biological significance of CD9 in a cellular context. The role of CD9 in the modulation of adhesive phenotypes have been investigated by the following approaches; 1) examination of CD9 expression profiles in cells at various stages of cancer progression to determine a correlation between CD9 expression levels and changes in cancer cell motility or tumor metastasis; 2) the ability and effects of antiCD9 mAbs in the modulation of cells phenotypes; and 3) modulation of CD9 expression and its effects on adhesive cell phenotypes. Due to extensive evidence linking CD9 expression to cell motility, CD9 is also known as Motility Related Protein (MRP-1). The studies that point to CD9 as an important regulator of cell motility are summarized below.

Altered expression of cell surface adhesion molecules in tumor cells is one of the major factors determining the metastatic potential. CD9 expression was shown to correlate well with the metastatic potential of various tumor cells. Miyake et al, 1996 first reported decreased expression of CD9 in the advanced breast cancer tissue when compared to CD9 expression in primary breast cancer tissue (112). Subsequently, a negative correlation between CD9 expression and metastatic potential of tumors has been reported in lung adenocarcinoma (113), esophageal (114) and oral squamous cell carcinomas (115), and small cell cancer of the lung (116).

In contrary to the above reports, expression of CD9 has also been shown to positively correlate with the metastatic potential of certain tumor cells. For example, in colon cancer cells CD9 expression was shown to be high at the primary site as opposed to 
the regions of metastasis (117). Sauer et al, 2003 reported a strong expression of CD9 on the cervical cancer cells near the sites of transendothelial invasion of these cells (118). A positive correlation between CD9 expression and late stage of cancer has also been reported in gastric carcinoma (119).

The role of anti-CD9 mAbs in the perturbation of cell motility has been investigated by several researchers. Masellis-Smith et al, 1990 first reported that several CD9 specific mAbs induced homotypic aggregation of the B-lymphoblastoid cell line, NALM-6 (120). Subsequently, the ability of CD9 mAbs to modulate cell motility has been studied in several model systems yielding contradictory results, where anti-CD9 mAbs have been shown to have both stimulatory and inhibitory effects on cell migration. Anti-CD9 mAbs inhibited migration of lung adenocarcinoma cells (121), Schwann cells (96), neuronal cell bodies (95), endothelial cells (91) colon cancer cells (117), transendothelial migration of melanoma cells (122), and parietal endodermal cell migration (123). Conversely, anti-CD9 mAbs promoted endometrial cancer cell migration (124), motility of melanocytes (125) and keratinocytes (126). Several researchers have also studied the role of CD9 in cell motility by altering the surface expression of CD9.

Most of the studies examined the phenotypic effects of ectopic expression of CD9 in cells lacking constitutively expressed CD9. Ikeyama et al, 1993 first reported that CD9 expression in the lung adenocarcinoma cell line MAC-10, suppressed cell motility (127). Subsequently, ectopic expression of CD9 was shown to inhibit motility (116) and metastatic potential (128) of small cell lung cancer. Ectopic expression of CD9 promoted cell migration in the CHO cells (129) and Raji cells (28). 
Additional evidence for the regulatory role of $\mathrm{CD} 9$ on cell motility came from the downregulation of endogenous CD9 expression by siRNA mediated gene silencing. Penas et al, 2005 reported that siRNA silencing of CD9 in melanoma cells inhibited cell motility (130). Recombinant EC2 regions and peptides corresponding to the EC2 region were also able to modulate cell motility. For example, a peptide corresponding to the CD9 EC2 amino acids 168-192 was shown to inhibit CD9 promoted haptotactic cell motility on FN (129) and a recombinant CD9-EC2 protein inhibited transendothelial migration of leukocytes (90).

The variations observed for the regulatory role of CD9 in different experimental approaches can be attributed to broad definition of the cell motility. Haptotaxis, chemotaxis and cell invasion are commonly referred as cell motility although independently these phenomena are governed by functionally distinct environmental cues. Furthermore, differences in the type/s of the extracellular matrix proteins used in the cell motility experiments introduce another variable in critically understanding the regulatory role of CD9 in cell motility.

In addition to participating in cell motility, CD9 was also shown to regulate cell fusion. Biological phenomenon dependent on CD9 mediated cell fusion include spermegg fusion, viral infectivity of cells, and formation multinucleated cells. One of the striking phenotypes of CD9 knockout mice (CD9 -/-) is the decreased ability of the ova derived from CD9 -/- mice to fuse with sperms resulting in reduced fertility (57-59). CD9 has been shown to associate with the integrin $\alpha 6 \beta 1$, a known receptor for the sperm protein fertilin (131). Subsequently, Kaji et al, 2002 have shown that that infertility of 
CD9 -/- oocytes can be reversed by exogenous expression of functional human CD9 mRNA or mouse CD81 mRNA sequences (132).

Additional evidence for CD9 in sperm-egg fusion came from the studies where anti-CD9 mAbs and recombinant proteins corresponding to $\mathrm{CD} 9 \mathrm{EC} 2$ regions were shown to inhibit sperm-egg binding and fusion process $(133,134)$. These studies have shown that CD9 EC2 region is essential for sperm-egg fusion. Interestingly, CD81 has recently been shown to participate in the sperm-egg fusion process. Rubinstein et al, 2006 reported that deletion of the $\mathrm{CD} 81$ gene in mice resulted in female infertility due to a defect in oocyte/ sperm fusion (67).

In vitro experimental results also suggest that $\mathrm{CD} 9$ is involved in muscle cell fusion and myotube formation (135), in osteoclastogenesis (136) and in the modulation of HIV-1 induced membrane fusion process (47). Tachibana et al, 1999 reported that antiCD9 mAbs perturb differentiation of myoblasts into myotubes (135). Overexpression of CD9 in a cultured myoblast cell line enhanced syncitia formation characteristic of the myotubes while antibody blockade delayed syncitia formation.

The cell fusion process is also important in the generation of osteoclasts. Osteoclasts are macrophage derived multinucleated cells that aid in bone remodeling. CD9 is highly expressed on the surface of osteoclasts and in vitro experimental results suggest that anti-CD9 mAbs can inhibit the formation of osteoclasts (136). An important regulatory role for $\mathrm{CD} 9$ in viral infections has recently been explored. Gordon-Alonso et al, 2006 reported that treatment of cells with mAbs for CD81 and CD9 or inhibition of CD81 or CD9 expression enhanced HIV-1 induced syncytia formation where as overexpression of CD81 or CD9 inhibited virus induced syncitia formation (47). 
From the above observations it is clear that the mechanisms utilized by CD9 in the modulation of adhesive cellular events involves complex processes that are influenced by the expression of other adhesion receptors, the definition of ECM and other experimental variables including proteinases and growth factors. Additionally, the results suggest that there is functional redundancy among tetraspanins. For example, most of the $\mathrm{CD} 9$ regulated cell fusion processes are also regulated by CD81. Among 32 other mammalian tetraspanins identified so far, CD9 has the closest amino acid sequence homology to CD81 (13) suggesting that structurally related tetraspanins may have significantly overlapping functions.

\subsection{CD9 Interactions with Cell Surface Molecules}

Tetraspanins are characterized by their unique ability to participate in extensive lateral interactions with other membrane proteins. CD9 was the first tetraspanin shown to associate with the other membrane proteins. Association of CD9 with platelet GPIIb/IIIa ( $\alpha$ IIb $\beta 3$ ) was first reported by Slupsky et al, 1989 (108). Subsequently most of the other tetraspanins have also been shown to associate with integrins (137). Figure 1.3 shows the CD9 binding partners identified in various cells.

After initial identification of CD9 interaction with integrin $\beta 3$ (108), several groups have reported association of CD9 with $\beta 1$ containing integrins (138). CD9 interactions with integrins are highly cell type specific. For example, a part from platelets, CD9 has not been shown to associate with $\beta 3$ integrins. CD9- $\beta 1$ association also appears to be highly specific. In various cell systems, CD9 was shown to be in complex with $\alpha 3 \beta 1, \alpha 4 \beta 1, \alpha 5 \beta 1, \alpha 6 \beta 1$, and $\alpha 6 \beta 4$ integrins. A summary of the 


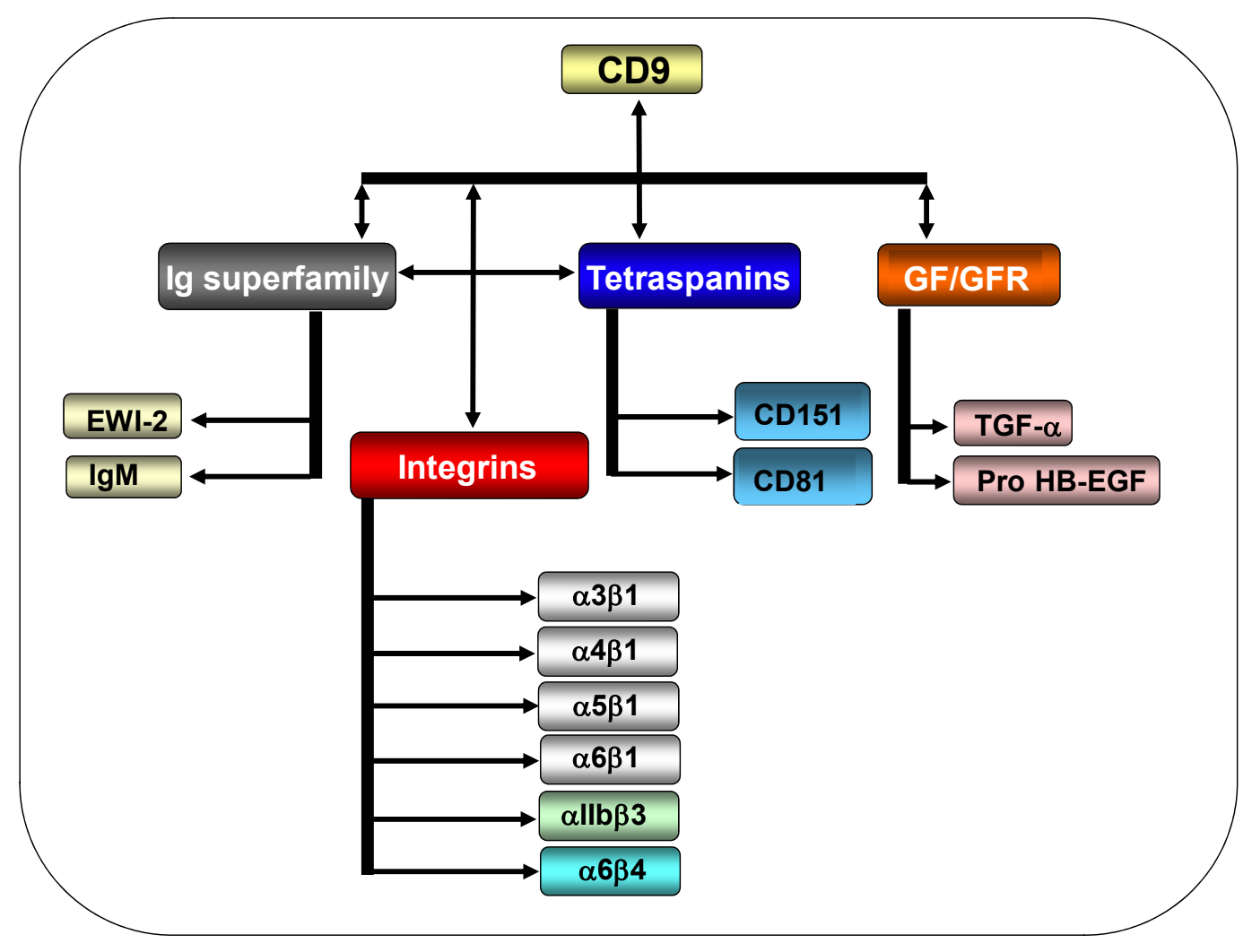

Figure 1.3 Molecular Interactions of CD9 with Membrane Proteins 
functional relevance of CD9 interactions with various $\beta 1$ integrins is presented below. Integrin $\alpha 3 \beta 1$ is the receptor for the ECM protein laminin (LN) (139). An association between CD9 and $\alpha 3 \beta 1$ was first reported on epidermal cells (140). CD9$\alpha 3 \beta 1$ complexes are often seen with other membrane proteins such as gangliosides (141) and pro-HB-EGF (142). CD9 in the $\alpha 3 \beta 1$-ganglioside complex was shown to modulate cell motility on LN (143).

Integrin $\alpha 4 \beta 1$ is the primary receptor for vascular cell adhesion molecule-1 (VCAM-1) $(144,145)$ and $\alpha 4 \beta 1$ can bind FN (146). $\alpha 4 \beta 1$ is expressed predominately on immune cells such as lymphocytes, eosinophils, and monocytes (144). As association between CD9 and $\alpha 4 \beta 1$ has been reported in the leukemic pre-B cell line, NALM-6, and the megakaryocytic cell line, HEL (138). Although both anti-CD9 and anti- $\alpha 4 \beta 1 \mathrm{mAbs}$ induce cell aggregation and inhibit cell migration in NALM-6 cells (138), whether CD9 directly modulates $\alpha 4 \beta 1$ interactions with its ligand, VCAM-1, is unknown. Integrin $\alpha 5 \beta 1$ is the primary receptor for $\mathrm{FN}(147)$.

Like most other CD9-integrin interactions, CD9- $\alpha 5 \beta 1$ complexes appear to be restricted to few cell types. In NALM-6 cells, CD9 was shown to associate with integrin $\alpha 5 \beta 1$ (138). Other cell lines where CD9 complexes with $\alpha 5 \beta 1$ include the trophoblasts (99) and the myocytes (135). However, the physiological significance of CD9- $\alpha 5 \beta 1$ interactions is not known.

CD9- $\alpha 6 \beta 1$ interactions have been reported in a variety of cells such as parietal endodermal cells (123), granulosa cells (100), oocytes (133), and Schwann cells (148). $\alpha 6 \beta 1$ in oocytes interacts with fertilin-beta (ADAM 2) on sperm cell surface (133). 
Although the functional implications of $\mathrm{CD} 9 / \alpha 6 \beta 1$ interactions is not clear, anti-CD9 mAbs have been shown to modulate Schwann cell (148), parietal endodermal cell migration (123), and block sperm-egg fusion $(133,134)$. A functional association between $\mathrm{CD} 9$ and $\mathrm{LN}$ binding integrin $\alpha 6 \beta 4$ has been reported. In cultured keratinocytes, CD9 was shown to be in complex with $\alpha 6 \beta 4$ (126) and anti-CD9 antibodies have been shown to modulate $\alpha 6 \beta 4$ dependent cell motility $(101,126)$ and spreading $(82)$.

Other members of tetraspanin family are an invariable component of CD9integrin complexes. The majority of CD9 complexes isolated from cells by immunoprecipitation (IP) contain one or more other tetraspanin member as well as integrins. The network of integrins and tetraspanins in biological membranes are termed a "tetraspanin web" (149). It has been hypothesized that the composition of the "tetraspanin web" can have a significant effect on how integrins function, which in turn determines cellular phenotype.

The most common tetraspanins seen in CD9 immune complexes include CD151, CD81, and CD82 $(82,150) . C D 151$ and CD81 are the only tetraspanins that retain association with integrins even under stringent detergent conditions such as Triton-X100 (22). Since CD9 interactions with integrins are disrupted by stringent detergents, it is thought that $\mathrm{CD} 151$ and $\mathrm{CD} 81$ are required to maintain CD9/integrin interactions.

Apart from adhesion molecules, so far, only a few growth factor family members have been shown to associate with CD9. The membrane-anchored heparin binding epidermal growth factor-like growth factor precursor (pro HB-EGF) is a transmembrane protein that belongs to the EGF superfamily (151). Pro-HB-EGF is a known diphtheria toxin receptor (DTR) (152). An association between CD9 and Pro-HBEGF has been 
reported, where CD9 EC2 was shown to be essential for its interaction with Pro-HB-EGF $(34,153)$. Expression of CD9 was also shown to upregulate the juxtacrine growth factor activity of Pro-HB-EGF (33).

Another growth factor family member that was shown to be in complex with CD9 is transforming growth factor- $\alpha$ (TGF- $\alpha)$. TGF- $\alpha$ is a member of epidermal growth factor (EGF) family (154) and structurally related to HB-EGF (155). TGF- $\alpha$, synthesized as a transmembrane protein, undergoes ectodomain cleavage to release the soluble form of TGF- $\alpha$ that activates EGF receptor (156). CD9 association with TGF- $\alpha$ was shown to decrease TGF- $\alpha$ susceptibility to ectodomain cleavage (157).

Major histocompatibility complex class II (MHC class-II) proteins are expressed on a wide variety of antigen presenting cells in the immune system (158). Dendritic cells (DC) are the antigen presenting cells that express both MHC class-II and CD9 $(48,159)$. In DC, CD9 has been detected in complex with MHC class-II molecules at the cell membrane (48). Engering et al, 2001 proposed that association of tetraspanins with the MHC class-II molecules is required for redistribution of MHC class II to the various compartments of DC. In support of this observation, the intracellular tetraspanin, CD63, was predominantly associated with intracellular MHC class-II (48) and cell surface CD9 was required for heterologous association of MHC class-II molecules with other membrane proteins (160).

CD9 has also been found in complex with Ig superfamily of proteins. EWI-2 is a recently identified member of the Ig superfamily of proteins that associates with tetraspanins CD9 and CD81 at high stoichiometry (29). Another Ig superfamily member 
found in complex with CD9 is IgM (32). Interactions between either CD9 or CD81 with EWI-2 were essential for recruiting of integrins in to tetraspanin/EWI-2 complexes (30).

$\mathrm{Fc}$ receptors $(\mathrm{FcR})$ are the cell surface molecules that recognize $\mathrm{Fc}$ portions of the immunoglobulins and mediate immunological phenomenon such as antibody mediated phagocytosis and complement activation. CD9 association with FcR has been reported in macrophages (161) and in platelets (162). Where CD9 was shown to be associated with FcR, anti-CD9 mAbs induce cross linking of FcR, leading to the activation of cells (161, 162).

\subsection{CD9 and Ligand Interactions}

In addition to extensively participating in lateral interactions with other membrane proteins, CD9 was also shown to serve as a receptor for both soluble and insoluble extracellular components. At least three extracellular proteins have been shown to act as ligands for CD9. Table 1.4 summarizes the list of CD9 ligand interactions that were reported to date. The first reported protein shown to bind CD9 was FN (129). FN is an extracellular matrix protein that is involved in several important biological processes such as embryogenesis, tissue remodeling/repair, and blood coagulation. Integrin $\alpha 5 \beta 1$ is the classical FN receptor and interaction of $\alpha 5 \beta 1$ with FN regulates the important cellular functions such as cell adhesion, migration and extracellular matrix assembly $(147,163$, 164).

FN is a $450 \mathrm{kDa}$ dimeric protein comprised of two identical $225 \mathrm{kDa}$ polypeptide chains joined by disulfide bonds (165). Structurally FN is composed of three types of repeating modules referred to as type1, type II, and type III repeats (166). Groups of 
Table 1.4 Ligand Interactions of CD9

\begin{tabular}{ccc}
\hline Ligand & Cell Type / Source of CD9 & Functions \\
\hline PSG17 & Monocyte and Macrophages & Immune Response \\
IL-16 & Mast Cells & Chemotactic Response \\
Fibronectin & Platelet Purified and & Cell Spreading, Motility, \\
& Recombinant CD9 & Pericellular FN Matrix \\
& & Assembly \\
\hline
\end{tabular}


modules are folded into six globular domains that bind various extracellular and cell adhesion molecules (167). Integrin $\alpha 5 \beta 1$ is the primary cell surface receptor for FN and recognition of FN by $\alpha 5 \beta 1$ requires the RGD (Arg-Gly-Asp) cell binding sequence in the type III module of FN $(168,169)$. In addition to cell surface integrins, $\mathrm{FN}$ also binds other ECM components such as collagen (170), glycosaminoglycans (171), and fibrin (172).

Utilizing platelet purified and recombinant CD9, Longhurst et al, 2002 reported that CD9 can interact with human plasma FN (129). Experimental evidence based on whole cell assays implicates that $\mathrm{EC} 2$ region of $\mathrm{CD} 9$ is important in binding to $\mathrm{FN}$. Experiments performed with FN fragment suggest that CD9 preferentially interacts with the $40 \mathrm{kDa}$ fragment of FN that includes heparin-binding domain Hep-2 and the IIICS domain but not with the $120 \mathrm{kDa}$ fragment that contains the cell attachment domain with RGD amino acid motif (173).

Pregnancy specific glycoproteins (PSG) are members of the carcinoembryonic antigen (CEA) family of proteins synthesized by the placenta (174). Secreted specifically during pregnancy, PSG family of proteins induces cytokine production by the monocyte/macrophage cells (175). PSG17 is the first soluble ligand that was reported to bind CD9 (176). Baculovirus expressed recombinant PSG17 was shown to selectively bind CD9 expressing cells but not CD151, CD81, CD82 expressing cells (176). Also, anti-CD9 mAbs specifically inhibited CD9 interactions with PSG17 suggesting that CD9/PSG17 binding is a direct cis interaction (177).

Ha et al, 2005 subsequently reported that binding of murine PSG17 to CD9 on monocytes can induce the production of anti-inflammatory cytokines such as IL-10, IL-6, 
and TGF- $\beta 1$ (178). Interestingly, mutations in CD9 regions required for sperm-egg fusion (amino acids: 173-175) also appear to be essential for PSG17-CD9 interaction (177).

Interleukins are molecules secreted by immune cells that mediate inflammatory response (179). Interleukin 16 (IL-16) is a lymphocyte chemo attractant that is a well established ligand for CD4 $(180,181)$. IL-16 is produced by variety of cells and acts as a major chemotactic stimulus for CD4 positive T-lymphocytes. Mast cells are components of connective tissue that participate in normal as well as abnormal immune response. Mast cells express high levels of tetraspanin CD9 but do not express CD4. Qi et al, 2006 reported that mast cell CD9 can serve as an accessory receptor for the IL-16 (182). IL-16 mediated chemotactic response of mast cells was blocked by anti-CD9 mAbs and IL-16 was shown to directly bind to CD9 expressing $\mathrm{CHO}$ cells.

The common feature of CD9 binding ligands is their ability to interact with the EC2 domain. Most of the monoclonal antibodies raised against CD9 bind the EC2 region of CD9, which explains the ability of these reagents to characteristically modulate CD9 mediated cellular effects. Additionally, synthetic peptides and recombinant proteins from the EC2 region also have profound effects on CD9 induced phenotypic changes.

\subsection{CD9 and Signal Transduction Pathways}

Signal transduction events mediate cellular responses to environmental cues. Signal transduction pathways are initiated by specific extracellular stimuli generated via cell surface molecules. For example, adhesion molecules such as integrins transduce intracellular signals upon engagement with the extracellular matrix components during cell adhesion (183). Recognition of extracellular stimuli by cells leads to activation of 
specific intracellular enzymes known as kinases. The final effect of signal transduction pathways such as regulation of gene transcription and cytoskeletal changes largely depend upon the type of signaling pathway that is activated in a cell.

The earliest evidence demonstrating the ability of CD9 to affect intracellular signaling events came from studies in human platelets, where anti-CD9 mAb, ALB6, was shown to induce calcium influx in platelets (111). Subsequently, several groups have reported a similar finding, where several other anti-CD9 mAbs were also shown to elicit $\mathrm{Ca}^{2+}$ influx in platelets $(184,185)$. Anti-CD9 $\mathrm{mAbs}$ have also been shown to activate p72 Syk in platelets (186), and enhance phosphoinositide turnover (102).

Evidence of CD9 participation in signal transduction pathways is not limited to anti-CD9 perturbation studies, as it has been shown that expression of CD9 in cultured cells can also modulate intracellular signaling. Shaw et al, 1995 showed that ectopic expression of CD9 in Burkitt's lymphoma cell line, Raji, induced tyrosine phosphorylation of several proteins in adhesion dependent manner (28). Klein-Soyer et al, 2000 have shown that adhesion of cultured endothelial cells to the anti-CD9 mAb coated substrata also induced protein tyrosine phosphorylation (91).

As with the CD9-integrin interactions, CD9 mediated signaling events also appear to be highly cell type specific as CD9 can activate a multitude of intracellular signal transduction pathways. This in part can be explained by the variations in the composition of the "tetraspanin web" from one cell to another (187). Table 1.5 summarizes the list of various signal transduction pathways that have been reportedly modulated by CD9.

Anti-human CD9 mAb, ALB-6, was shown to induce apoptosis in tumor cells (188). Shc is an adapter protein that upon tyrosine phosphorylation activates multiple 
intracellular signaling pathways (189). In the gastric cancer cell line, MKN-28, treatment of cells with ALB-6 antibody enhanced tyrosine phosphorylation of p46 isoform of Shc, an event that lead to activation of c-Jun $\mathrm{NH}_{2}$ - terminal kinase/stress-activated protein kinase (JNK/SAPK) and the p38 MAPK pathways.

JNK/MAPK pathways are activated on expression of CD9 in human melanoma cells (190). In melanoma cells, expression of CD9 was shown to induce matrix metalloprotenase-2 (MMP-2) expression in a JNK/p38 MAPK dependent manner (190). Other components of MAPK pathway are reportedly activated by CD9. For example, using anti-CD9 perturbation, p44/42 MAPK but not p38MAPK was shown to be important in CD9 dependent osteoclast differentiation (136).

Wnt signaling pathway is essential for normal cellular development and mutations of molecules in this pathway are implicated in several cancers (191). Expression of CD9 in tumor cell lines HT1080 and A549 was shown to downregulate expression of Wnt family of proteins Wnt1, Wnt2b1 and Wnt5a (192) and the Wiskott-Aldrich syndrome protein (WASP), WAVE-2 (193). Expression of CD9 in HT1080 cells also altered the subcellular localization of the cytoskeleton modifying proteins Arp2 and Arp3 which was dependent on WAVE-2 but not Wnt members (193). The functional significance of Wnt signaling pathway in CD9 mediated cellular effects remains to be addressed.

In addition to the above reported observations, CD9 and other tetraspanins were shown to associate with the lipid kinase, phosphatidylinositol-4 kinase (PI-4K) ((20). Although this finding suggests that tetraspanins may recruit PI-4K to the membrane proximal regions, the biological relevance of this association is not known. Focal adhesion kinase (FAK) is both phosphorylated and activated downstream of integrins 
Table 1.5 Intracellular Signaling Pathways Modulated by CD9

\begin{tabular}{|c|c|c|}
\hline Molecule & Cells & Effects \\
\hline PI-4 Kinase & - & - \\
\hline P46Shc & Cancer Cells & \\
\hline Wnt1, Wnt2b1 & Fibrosarcoma & - \\
\hline and Wnt5a & Cells & \\
\hline P44/42 MAPK & Osteoclasts & Osteoclastogenesis \\
\hline \multirow[t]{3}{*}{ P38 MAPK } & Melanoma Cells & Regulates Matrix \\
\hline & & Metalloprotease \\
\hline & & Expression \\
\hline \multirow[t]{2}{*}{ FAK } & Vascular Smooth & Cell Motility \\
\hline & Muscle Cells & \\
\hline WAVE-2 & Fibrosarcoma Cells & Cell Motility \\
\hline \multirow[t]{2}{*}{$\mathrm{Ca}^{2+}$} & Platelets & Platelet Activation and \\
\hline & & Aggregation \\
\hline
\end{tabular}


leading to stimulation of other intracellular signaling pathways such phosphatidylinositol3 kinase (PI-3K) (194). In vascular smooth muscle cells, CD9 was shown to modulate tyrosine phosphorylation of FAK (92).

Based on the above observations, the following conclusions can be drawn with regard to overall role of CD9 in signal transduction pathways. Since most of the reports utilize mAbs to interfere with CD9's function and because mAbs can be either stimulatory or inhibitory in nature, it is unknown whether CD9 is actually stimulatory or inhibitory with respect to specific signaling pathways. The findings also suggest that in a given cell, CD9 has the potential to modulate multiple signaling pathways. Also, it is unclear whether important CD9 mediated biological phenomenon such as cell migration directly depends on these signaling pathways. 


\section{Chapter 2: Study Objectives}

\subsection{Background}

$\mathrm{CHO}$ cells were originally described as an in vitro model system for their unique characteristic of genomic stability in long-term culture (195). Since then researchers have extensively used $\mathrm{CHO}$ cells as a model system for characterizing a variety of novel proteins (196-199). The effects of stable expression of human CD9 on CHO cell phenotype have been thoroughly characterized and documented in our laboratory. The major phenotypic changes induced on expression of human CD9 in CHO cells include altered cell morphology, spreading, adhesion, haptotactic cell motility, and pericellular FN matrix assembly $(129,173,182,200)$.

Morphologically CD9- CHO cells are very distinct from the Mock transfected cells (Mock-CHO) (173). CD9- CHO cells appear as polygonal shaped cells as opposed to characteristic spindle shape of Mock-CHO cells or the naïve $\mathrm{CHO}$ cells. In addition, CD9-CHO exhibited significantly increased cell spreading on FN (173) when compared to Mock-CHO cells. Comparative analysis of the CD9 induced spread phenotype on fragments of FN showed that a $40 \mathrm{kDa}$ fragment of FN that includes the HEP2/IIICS regions was sufficient to induce this phenotype (173).

Another significant phenotypic change induced in $\mathrm{CHO}$ cells upon expression of human CD9 is upregulation of haptotactic cell motility on FN (129). In vitro Transwell migration assays showed that CD9-CHO cells had a 3-4 fold higher migration rate than Mock-CHO cells. The promigratory effect of CD9 was specific for FN and was not seen with other extracellular matrix proteins such as fibrinogen (FG) (129). 
To define the critical regions on CD9 required for enhanced cell migration on FN, $\mathrm{CHO}$ cell lines expressing $\mathrm{CD} 9$ truncation mutants in the $\mathrm{EC} 2$ region were generated $(129,173,200)$. Analysis of these cell lines showed that the EC2 region was sufficient and necessary for promoting cell migration on FN (129). Furthermore, a peptide corresponding to the EC2 region (a.a 168-192) blocked CD9 induced haptotactic cell motility on FN (129). Similarly, recombinant fragments corresponding to the CD9 EC2 region were also shown to inhibit transendothelial migration of leukocytes (90). Also, as mentioned earlier both bacterially expressed and platelet purified CD9 were able to bind purified FN (129).

In addition to profoundly influencing cell spreading and cell motility, CD9 expression also modulates the extent of pericellular FN matrix assembly in $\mathrm{CHO}$ cells (173). As seen in studies of haptotactic motility, the EC2 region of CD9 was shown to be critical for modulating pericellular FN matrix assembly in $\mathrm{CHO}$ cells. These studies demonstrated that CD9 modulated adhesive phenotypes in which integrin $\alpha 5 \beta 1$ directly participates. $\mathrm{CHO}$ cells express the integrin $\alpha 5 \beta 1$ endogenously where it acts as the primary FN receptor (147). Interestingly, a function blocking $\mathrm{mAb}$ specific for hamster $\alpha 5 \beta 1$ abolished CD9 induced CHO cell spreading on FN (201).

Cumulatively, these earlier studies suggest that CD9 acts in concert with $\alpha 5 \beta 1$ to influence adhesive cell phenotypes in response to FN. The precise molecular mechanisms of potential CD9- $\alpha 5 \beta 1$ cooperation are as yet unclear and are a subject of current investigation. Both CD9 and integrin $\alpha 5 \beta 1$ can activate intracellular signaling pathways. Integrin mediated signal transduction events driving cell motility have been well characterized and documented (202). In contrast, the influence of CD9 on signal 
transduction pathways responsible for specific adhesion dependent cell phenotypes still remains elusive. In order to address these previously unanswered questions, our current study aims to gain insight into novel aspects of CD9 biology.

\subsection{CD9 Mediated Signaling Pathways in Cell Motility}

In order to gain insight into the molecular mechanisms of CD9 mediated cell motility, one of our objectives was to investigate the intracellular signal transduction pathways involved in CD9 mediated haptotactic cell motility. Given the complexity of CD9-integrin interactions, a wide range of intracellular signaling pathways emerge as potential candidates for CD9 mediated cell migration. For example, activation of adhesion molecules can activate a number of intracellular kinases such as MAPK (203), PI-3K (204), and focal adhesion kinase (FAK) (194) that are important regulators of cell motility. Adapter proteins such as p130Cas have also been shown to modulate cell motility downstream of integrins (205) and kinases such as PKC have been reported to associate directly with tetraspanins (38).

In this study, we systematically examined the role of several of these candidate molecules in CD9 mediated haptotactic cell motility to FN. A number of cell permeable, small molecule inhibitors are available to target these signaling molecules in a highly specific manner. The first approach was to test the effects of these compounds in CD9 mediated haptotactic cell motility. Where possible, molecular strategies such as dominant/negative transfections, siRNA mediated gene silencing will be employed to further define the role of individual signaling pathways responsible for CD9 mediated cell motility. 


\subsection{Role of Integrins in CD9 Mediated Cell Motility}

Since, CD9 and other tetraspanins participate in extensive lateral interactions with integrins (137), CD9 may play a role in the regulation of integrin conformational states. Information regarding the ability of tetraspanins to participate in mechanisms modulating integrin conformation is limited. Recent studies have shown that tetraspanin CD151 can stabilize the active conformation of integrin $\alpha 3 \beta 1$ (206). CD9 complex with platelet GPIIb/IIIa has been shown to be dependent upon the conformational state of GPIIb/IIIa (110). Although CD9 associates with integrin $\beta 1$, it is not known whether CD9 can modulate $\beta 1$ conformation states.

Integrins exist in two different conformational states $(25,207,208)$. Based on structural studies, it has been hypothesized that the head domain of integrins can exist in a bent conformation that extends upon ligand binding. Also, integrin mediated cellular effects are thought to directly correlate with the active conformation of the molecule $(209,210)$. In addition to the ligand binding, integrins can be activated by divalent cations such as $\mathrm{Mn}^{2+}$ and $\mathrm{Ca}^{2+}(211-213)$.

Therefore, the second major focus of the study was to explore CD9 interactions with integrins and to study the possible role of CD9 in modulating integrin conformation. Using cultured cells and platelets as model systems, we investigated CD9 interaction with $\beta 1$ and $\beta 3$ integrins. We also proposed to examine the physical relationship between CD9 and $\alpha 5 \beta 1$. Additionally, the functional significance of CD9- $\alpha 5 \beta 1$ interactions in CD9 mediated haptotactic cell motility was investigated with the available function blocking mAbs. Finally, we aimed to determine the role of CD9 in modulation of $\beta 1$ integrin conformation and activation. 
Figure 2.1 is a schematic representation of the potential signal transduction mechanisms that are implicated in CD9 mediated cell motility. In summary, modulation of integrin conformation and therefore function by CD9 can regulate intracellular signal transduction cascades emanating from cell-ECM ligand interactions. The proposed signaling molecules (PI-3K, PKC, MAPK, p130Cas, and FAK) under investigation have been well documented to participate in adhesion molecule mediated signal transduction cascades. 


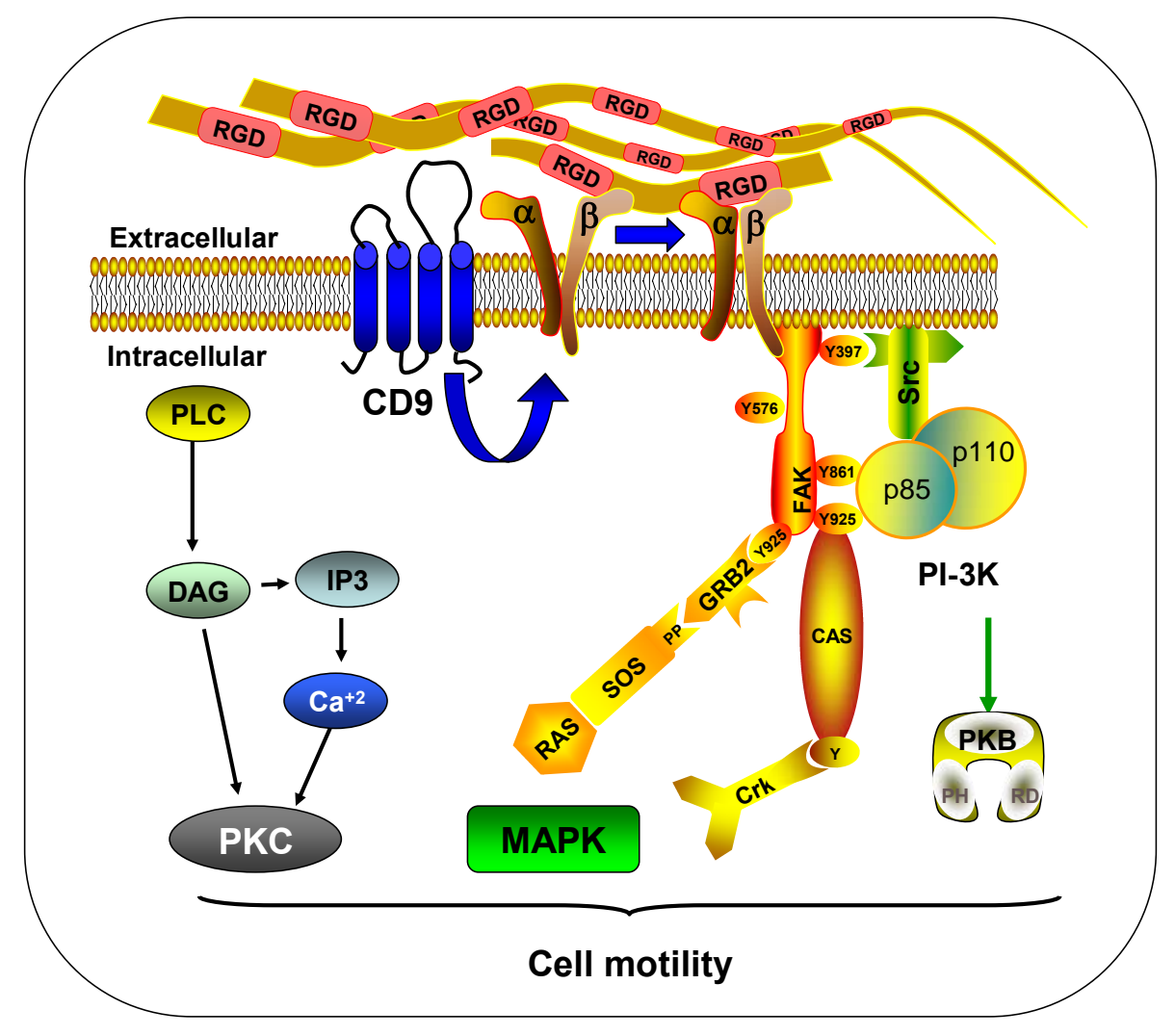

Figure 2.1 Hypothesized Mechanisms of CD9 Mediated Cell Migration

PLC: Phospholipase C, DAG: Diacyl glycerol, IP3: Inositol 3 phosphate, PKB: Protein kinase B, RGD: Minimal domain in ECM proteins that binds integrins, $\alpha, \beta$ : Subunits of functional integrin molecule, FAK: Focal adhesion kinase, MAPK: Mitogen-activated protein kinase, PI-3K: Phosphatidylinositol-3 kinase, Y: Tyrosine residue of signaling molecules. 


\section{Chapter 3: Experimental Procedures}

\subsection{Antibodies and Reagents}

All the chemical reagents, unless otherwise specified, were purchased from Sigma-Aldrich (St. Louis, MO). Human plasma FN, RPMI, DMEM, Trypsin-EDTA and geneticin were obtained from Invitrogen (Carlsbad, CA). Pharmacological inhibitors wortmannin, LY294002, Gö6976, and PD98059 were purchased from Calbiochem (San Diego, CA). The monoclonal anti-CD9 antibody, mAb7, has been described previously (109). Anti-rat CD9 (RPM.7), anti-mouse CD9 (KMC.8) and anti-rat $\alpha 5$ (HM $\alpha 5-1)$ antibodies were from BD Pharmigen (San Diego, CA). Anti-human $\beta 1$ integrin mAb (TS2/16) was a kind gift from Dr. Xin Zhang.

Anti-integrin $\beta 1$ (Clone-B44), and anti-actin mAb (clone C4) were purchased from Chemicon International (Temecula, CA). Anti rat- $\beta 1$ (HM $\beta 1-1)$, hamster IgG isotype control, and goat anti-hamster FITC antibodies were from Biolegend (San Diego, CA). Monoclonal antibodies against platelet GPIIb/IIIa complex (10E5, C3) and IIIa or $\beta 3$ (AP3) and have been previously described $(214,215)$. The mAb 10E5 was a gift from Dr. Barry Coller and AP3 was kindly provided to us by Dr. Peter Newman. Anti-human $\beta 1$ integrin antibody that reacts with rat $\beta 1$ integrin (clone-18), anti-p130Cas mAb (clone 21), anti-focal adhesion kinase mAb (clone 77/FAK) and horseradish peroxidase (HRP) conjugated anti-phosphotyrosine (Clone-RC20) mAbs were from BD Transduction Laboratories (San Diego, CA).

Rabbit polyclonal anti- $\alpha 5$ antibody (H-104), anti-CD9 antibody (C-4), anti- $\beta 3$ (C10), p130Cas siRNA duplex and the control siRNA duplex were from Santa-Cruz 
Biotechnology (Santa Cruz, CA). Anti-rat $\alpha 1$ (clone 3A3), and anti-rat $\beta 3$ (clone F11) antibodies were from Serotec (Oxford, UK). Anti-phospho Akt S473 (9271s) and total Akt (9272) antibodies were from Cell Signaling (Beverly, MA). Anti-hamster $\alpha 5 \beta 1$ (PB1) was purchased from Developmental Studies Hybridoma Bank (Iowa City, IA). Rabbit polyclonal anti-PI-3K (p85) antibody was from Upstate Biotechnology (Lake Placid, NY). Anti-GFP antibody (JL-8) and BD-Adeno-X virus purification kit were purchased from BD Biosciences (Bedford, MA). The PI-3K dominant/negative plasmid $(\Delta \mathrm{p} 85)$ was a kind gift from Dr. Larry Pfeffer.

Transwell polycarbonate filters $(8.0 \mu \mathrm{m}$ pore size $)$ for migration assays were purchased from Nunc International (Rochester, NY). Permount solution was purchased from Fisher Scientific. All the secondary antibodies for Western blot and the ECL substrate kits were from Amersham Biosciences (Piscataway, NJ). Protein G-Plus Protein-A agarose beads were from Oncogene (San Diego, CA). Non fat dry milk (NFDM) was purchased from Nestle. Restore ${ }^{\mathrm{TM}}$ Western blot stripping buffer was purchased from Pierce (Rockford, IL).

\subsection{Cell Culture Methods}

The Chinese hamster ovary cell line CHO-K1, purchased from American Type Culture Collection (ATCC) was used for these studies. Mock transfected CHO (Mock$\mathrm{CHO}$ ) cells and a stable clone of CD9-CHO (A6) cells have been previously described (200). Mock and A6 cells were routinely cultured in RPMI-1640 medium supplemented with $10 \%$ fetal bovine serum (FBS) and $0.75 \mathrm{mg} / \mathrm{ml}$ of the selection agent Geneticin (RPMI Growth Medium). 
Smooth muscle cells isolated from thoracic aortae of Sprague-Dawley rats by enzymatic dissociation were provided to us by Dr. Gadiparthi N. Rao. Isolated smooth muscle cells were cultured in Dulbecco's Modified Eagles Medium (DMEM) supplemented with $10 \% \mathrm{FBS}, 100$ units $/ \mathrm{ml}$ of penicillin and $100 \mu \mathrm{g} / \mathrm{ml}$ of streptomycin (DMEM Growth Medium). After expansion, cells were frozen in liquid nitrogen in the DMEM medium containing 20\% FBS and 10\% dimethyl sulfoxide (DMSO). For culture, the frozen vial of cells are quickly thawed and added to culture dish containing prewarmed DMEM Growth Medium. Mouse embryonic fibroblasts (MEF) and Human embryonic kidney (HEK-293) cells were purchased from ATCC and were cultured in DMEM Growth Medium as described above.

The human fibrosarcoma cell line (HT1080) was cultured in DMEM Growth Medium. For generation of CD9 stable transfectants of HT1080 cells, the following protocol was used. Approximately $2.5 \times 10^{7} \mathrm{HT} 1080$ cells suspended in $300 \mu \mathrm{l}$ of ice cold phosphate buffered saline (PBS) were mixed with $50 \mu \mathrm{g}$ of $\mathrm{pRc} / \mathrm{CMV}$ plasmid containing human CD9 cDNA (77) and incubated on ice for 10 min. Pulse electroporation was performed at $0 \mathrm{Ohms}, 300 \mathrm{~V}$ and $950 \mu \mathrm{F}$ setting and immediately after electroporation; cells were transferred onto ice and rested for $10 \mathrm{~min}$ at room temperature.

The electroporated cells were transferred into culture flasks containing DMEM supplemented with $10 \% \mathrm{FBS}$ and incubated in humidified $\mathrm{CO}_{2}$ incubator at $37^{\circ} \mathrm{C}$. Electroporation with empty vector ( $\mathrm{pRc} / \mathrm{CMV}$ ) were similarly performed to generate Mock-HT1080 cells. Since pRc/CMV plasmid contained G418 as a eukaryotic selection marker, $48 \mathrm{hrs}$ after transfection, the culture medium was supplemented with $0.75 \mathrm{mg} / \mathrm{ml}$ of Geneticin. Successful expression of CD9 in the transfected cells was confirmed by 
flow cytometry (FCM) with a monoclonal antibody specific for CD9 (mAb7).

Subsequently, the population of cells with highest expression of human CD9 (top 5\%) was sorted by fluorescent-labeled cell sorting using mAb7.

\subsection{Flow Cytometry}

FCM was routinely performed to detect the surface expression of $\mathrm{CD} 9$ on $\mathrm{A} 6$ cells. For these experiments, confluent Mock or A6 cells were seeded at a density of $2.5 \times 10^{6}$ cells $/ 75 \mathrm{~cm}^{2}$ flask 24 hrs prior to FCM. Twelve to eighteen hrs after seeding, cells were harvested by trypsinization for $2 \mathrm{~min}$ at $37^{\circ} \mathrm{C}$ and counted by trypan blue exclusion. Cells were then suspended in RPMI labeling media (RPMI-1640 containing $5 \%$ goat serum) as $5 \times 10^{5}$ cells $/ \mathrm{ml}$ and incubated on ice for $1 \mathrm{hr}$. Cells were subsequently incubated in the primary antibody (mAb7; $4 \mu \mathrm{g} / \mathrm{ml})$ for $1 \mathrm{hr}$ on ice. The unbound antibody was removed by washing the cells three times with ice cold PBS and cells were incubated in the secondary antibody diluted in labeling media (goat-anti-mouse FITC conjugate; $5 \mu \mathrm{g} / \mathrm{ml}$ ) for $1 \mathrm{hr}$ on ice. After secondary antibody incubation, cells were washed 3 times in PBS and suspended in $0.5 \mathrm{ml}$ of PBS and data were acquired using FACS Calibur flow cytometer (Becton-Dickinson, Mountain View, CA) equipped with Cell Quest Pro software.

For FCM analysis utilizing the adenoviral transduced Rat Aortic Smooth Muscle (RASM) cells, experiments were performed $72 \mathrm{hrs}$ after transduction. Briefly, adenoviral transduced RASM cells were harvested by trypsinization for $1 \mathrm{~min}$ at $37^{\circ} \mathrm{C}$ and the harvested cells were suspended in DMEM labeling medium (DMEM containing 5\% goat 
serum) for $1 \mathrm{hr}$ on ice. Subsequent steps for primary, secondary antibody incubation and data analysis were performed as described above.

For FCM experiments performed for the detection of active conformation of $\beta 1$ integrin, the following procedure was used. Lyophilized integrin activating peptide RGDS and the negative control peptide RGES were reconstituted in PBS and $10 \mathrm{mM}$ stock solutions were aliquoted and stored at $-80^{\circ} \mathrm{C}$. Mock or $\mathrm{A} 6$ cells were cultured as described above. Harvested cells were suspended in RPMI labeling media as $5 \times 10^{5}$ cells $/ \mathrm{ml}$ and incubated for $1 \mathrm{hr}$ on ice.

In order to activate $\beta 1$ integrin, cells were incubated with $1 \mathrm{mM}$ RGDS or RGES peptides for $30 \mathrm{~min}$ at $37^{\circ} \mathrm{C}$. Subsequent to activation of integrin with the peptides, cells were incubated with the $5 \mu \mathrm{g}$ of $\beta 1$ integrin conformation sensitive antibody (B44) and incubated on ice for $45 \mathrm{~min}$. The unbound antibody was removed by washing cells three times with PBS and the cells were incubated with goat anti-mouse-FITC (GAM-FITC) conjugated secondary antibody. Cells were further processed as described in the earlier sections.

\subsection{Immunoprecipitation and Western Blot Analysis of Platelet Antigens}

All the steps for platelet isolation were carried out at room temperature. Venous blood was drawn from healthy, consented adult volunteers in to anticoagulant ACD (85 mM sodium citrate, $100 \mathrm{mM}$ dextrose, $70 \mathrm{mM}$ citric acid, $\mathrm{pH}$ 4.6) and platelet-rich plasma (PRP) was separated by centrifugation at $100 \mathrm{Xg}$ for $20 \mathrm{~min}$. Platelets were further isolated from PRP by centrifugation at $800 \mathrm{Xg}$ for $10 \mathrm{~min}$. The isolated platelets were 
gently resuspended in a buffer containing $10 \mathrm{mM}$ sodium citrate, $30 \mathrm{mM}$ dextrose and $120 \mathrm{mM}$ sodium chloride, $\mathrm{pH} 6.5$ (CGS buffer).

Platelets were washed twice in CGS buffer prior to lysis in Brij-98 lysis buffer (Table 3.1). Platelet proteins were solubilized in the Bri-98 lysis buffer for $20 \mathrm{~min}$ at $4^{\circ} \mathrm{C}$ and the resulting lysates were clarified by centrifugation at $14,000 \mathrm{rpm}$ for $10 \mathrm{~min}$ at $4^{\circ} \mathrm{C}$ and protein concentration was determined using the detergent compatible ${ }^{D c}$ protein assay kit (Bio-Rad, Hercules, CA).

For IP, lysate volume equivalent to $2 \mathrm{mg}$ protein for each sample was made to 1 $\mathrm{ml}$ with Bri-98 lysis buffer. For preclearing, samples were incubated with $75 \mu \mathrm{l}$ of protein A plus protein $\mathrm{G}$ agarose beads with agitation for $1 \mathrm{hr}$ at $4^{\circ} \mathrm{C}$. Lysates were then centrifuged at 14,000 rpm for $1 \mathrm{~min}$, beads discarded and the supernatants were transferred in to fresh tubes. The precleared lysates were then incubated with $5 \mu \mathrm{g}$ of the $\mathrm{mAb}$ specific for the platelet antigen (Table 3.2) and incubated for $1 \mathrm{hr}$ at $4^{\circ} \mathrm{C}$ on IP rocker.

As a negative control, lysates were also incubated with the isotype-matched $\mathrm{mAb}$ (MOPC-21). Immune complexes were captured by incubating with $75 \mu \mathrm{l}$ of protein A plus protein $\mathrm{G}$ agarose beads for $1 \mathrm{hr}$ at $4^{\circ} \mathrm{C}$ with agitation. The beads were washed 3-4 times with the Brij-98 lysis buffer, and after the last wash, $20 \mu 1$ lysis buffer was left with the beads. Immune complexes were eluted from the beads by boiling the samples in Laemmli sample buffer for $10 \mathrm{~min}$.

The eluted proteins were resolved on 5-20\% gradient of sodium dodecyl sulfatepolyacrylamide gel (SDS-PAGE). For Western blot analysis, proteins were transferred onto Immobilon-P membrane (Millipore, Billerica, MA) by electro-elution at a constant 
Table 3.1 Composition of Brij-98 Lysis Buffer

\begin{tabular}{ccc}
\hline Reagent & Stock & Final \\
\hline Brij-98 & $10 \%$ & $1 \%$ \\
Hepes & $1 \mathrm{M}$ & $10 \mathrm{mM}$ \\
$\mathrm{NaCl}$ & $3 \mathrm{M}$ & $200 \mathrm{mM}$ \\
$\mathrm{MgCl}_{2}$ & $1 \mathrm{M}$ & $5 \mathrm{mM}$ \\
$\mathrm{NaF}$ & $0.5 \mathrm{M}$ & $2 \mathrm{mM}$ \\
$\mathrm{Na}_{4} \mathrm{P}_{2} \mathrm{O}_{7}$ & $100 \mathrm{mM}$ & $10 \mathrm{mM}$ \\
$\mathrm{Na}_{3} \mathrm{VO}_{4}$ & $200 \mathrm{mM}$ & $200 \mu \mathrm{M}$ \\
\hline
\end{tabular}


Table 3.2 Antibodies Used for the Characterization of CD9-GPIIb/IIIa Complex

\begin{tabular}{cccc}
\hline Platelet Antigen & Antibody for IP & $\begin{array}{c}\text { Primary Antibody } \\
\text { (Dilution) }\end{array}$ & $\begin{array}{c}\text { Secondary Antibody } \\
\text { (Dilution) }\end{array}$ \\
\hline GPIIb/IIIa & $10 \mathrm{E} 5$ & Anti-GPIIb, C-3 & SAM-HRP \\
& & $(1: 10,000)$ & $(1: 50,000)$ \\
B3 integrin & AP3 & Anti- $\beta 3, \mathrm{C}-20$ & MAG-HRP \\
& & $(1: 500)$ & $(1: 50,000)$ \\
CD9 & Ant & Anti-CD9, C-4 & SAM-HRP \\
& & $(1: 500)$ & $(1: 5,000)$ \\
\hline
\end{tabular}


current setting of $700 \mathrm{mAmp}$. After the transfer, membranes were blocked for $6 \mathrm{hrs}$ at $4^{\circ} \mathrm{C}$ in TBST (20 mM Tris, $200 \mathrm{mM} \mathrm{NaCl}, 0.1 \%$ Tween-20) containing 5\% non-fat dry milk (NFDM). Further to this, blots were incubated in primary antibody diluted in TBST $/ 5 \%$ NFDM for $12-18 \mathrm{hrs}$ at $4^{\circ} \mathrm{C}$.

The unbound antibody was removed by washing the blots (5 times) with TBST for $10 \mathrm{~min}$ at room temperature. After the final wash, the membranes were incubated in the secondary antibody solution for $1 \mathrm{hr}$ at room temperature. Finally, membranes were washed 5 times $(10 \mathrm{~min} /$ wash $)$ in TBST and developed with enhanced chemiluminescence (ECL) substrate. Table 3.2 shows the list of antibodies and their dilutions used for Western blot analysis of the platelet antigens.

\subsection{Generation and Purification of CD9-Adenovirus}

The E1 region deleted adenoviral shuttle vector and pacAd5 9.2-100 plasmid were kindly provided to us by Dr. Aviv Hassid. Using these reagents, recombinant adenovirus expressing human CD9 (Ad-CD9) was generated as follows. The EcoR1 and Xba1 double digested fragment containing full-length human cDNA gene was excised from the plasmid pRc/CMVp24/CD9 (77) and was subcloned into EcoR1 and Xba1 sites of E1 deleted adenoviral shuttle vector. The resulting adenoviral shuttle vector was cotransfected into HEK293 cells with pacAd5 9.2-100, an adenoviral type5 backbone genome plasmid to generate Ad-CD9 by in vivo recombination. The Ad-CD9 construct is a replication-deficient, recombinant adenoviral vector capable of expressing full-length human CD9. As a negative control for transduction experiments, adenovirus containing LacZ gene (Ad-LacZ) was generated as described earlier (216). 
For purification, adenovirus was amplified in a E1 complementing HEK 293 cell line with a BD-Adeno-X virus purification kit according to the manufacturer's protocol. Briefly, low passage number HEK-293 cells cultured in DMEM medium supplemented with $10 \%$ FBS. The cells were infected for 90 min with the stock virus at a virus concentration of one multiplicity of infection (MOI). MOI is defined as a ratio between numbers of infectious virus particles to the number of target cells. After typical cytopathic effect (cells detach from the substratum and form grape like clusters) was observed, cells were harvested, and virus was purified utilizing a BD-Adeno-X virus isolation kit. After purification, adenoviral stock solutions were aliquoted and stored at $80^{\circ} \mathrm{C}$ for future use. The titer for the virus was estimated by soft-agar plaque assay as described previously (216).

For the transduction of rat aortic smooth muscle cells (RAMS), cells were first grown to $\sim 80 \%$ confluence in $10 \mathrm{~cm}$ culture dishes. The volume of Ad-CD9 or Ad-LacZ virus stock solutions equivalent to $100 \mathrm{MOI}$ was diluted in $1 \mathrm{ml}$ DMEM medium. The RASM cell growth media was removed, and replaced with $1 \mathrm{ml}$ of the virus solution in DMEM medium dropwise. The infected cells were then incubated at $37^{\circ} \mathrm{C}$ in $5 \% \mathrm{CO}_{2}$ humidified incubator for 90 min with manual rocking every 15 min. Negative control RASM cells treated with $1 \mathrm{ml}$ of DMEM alone. After 90 min post infection, the culture were supplemented with $9 \mathrm{ml}$ of DMEM medium and returned to the incubator. Subsequent analysis of cells was done at $72 \mathrm{hrs}$ post infection. 


\subsection{Immunoprecipitation and Western Blot Analysis of Cultured Cells}

For HT1080 cells and the MEF, culture monolayer's were washed two times with ice cold PBS (10 ml/dish for each wash) to remove the traces of fetal bovine serum (FBS). The cells were then scraped into Brij-98 lysis buffer and proteins were solubilized for $20 \mathrm{~min}$ at $4^{\circ} \mathrm{C}$. The resultant lysates were clarified by centrifugation at $14,000 \mathrm{rpm}$ for $10 \mathrm{~min}$ at $4^{\circ} \mathrm{C}$ and supernatants were transferred into fresh tubes and were stored at $80^{\circ} \mathrm{C}$.

For IP of proteins from MEF cell lysates, $1 \mathrm{mg}$ protein for each sample was made to $1 \mathrm{ml}$ with Bri-98 lysis buffer. The lysates were then incubated with $5 \mu \mathrm{g}$ anti-CD9 (KMC.8), anti- $\alpha 5$ (HM $\alpha 5-1)$, and anti- $\beta 1$ (HM $\beta 1-1) \mathrm{mAbs}$ for $1 \mathrm{hr} 4^{\circ} \mathrm{C}$ with agitation. As a negative control, lysates were incubated either with rat $\operatorname{IgG}$ (isotype control for KMC.8) or the hamster IgG (isotype control for HM $\alpha 5-1, \mathrm{HM} \beta-1$ ). Subsequently, immune complexes were captured with $75 \mu \mathrm{l}$ of protein A plus protein $\mathrm{G}$ agarose beads for $1 \mathrm{hr}$ at $4^{\circ} \mathrm{C}$ with agitation. The beads were washed 3-4 times with the Brij-98 lysis buffer, and after the last wash, $20 \mu \mathrm{l}$ lysis buffer was left with the beads. Immune complexes were eluted from the beads by boiling the samples in Laemmli sample buffer for $10 \mathrm{~min}$.

The eluted proteins were resolved on 5-20\% SDS-PAGE and Western blot was performed as described above. The presence of $\alpha 5$ in the immunoprecpitates was detected with a polyclonal antibody against $\alpha 5$. IP experiments with HT-1080 cells were essentially performed by similar protocol except that mAb7 and TS2/16 antibodies were used to immunoprecipitate $\mathrm{CD} 9$ and $\beta 1$, respectively. 
For IP experiments RASM cells were lysed in Brij-98 lysis buffer. For each IP reaction, $1 \mathrm{mg}$ of lysate was precleared with protein-G plus protein-A agarose beads for 1 $\mathrm{hr}$ at $4^{\circ} \mathrm{C}$. Targeted proteins from precleared lysates were immunoprecipitated with $5 \mu \mathrm{g}$ of antigen specific $\mathrm{mAb}$ and the respective isotype matched control mAbs. Immunecomplexes were washed four times with Brij-98 lysis buffer and samples were boiled in Lammeli sample buffer before loading onto 5-20\% SDS-Page. Subsequent analysis of proteins by Western blot was carried out as described above. Table 3.3 summarizes the list of antibodies used in these experiments.

\subsection{Determination of Akt Phosphorylation}

Whole cell lysates were prepared from cultured cells either held in suspension or plated on to FN coated culture dishes. For coating, four $\mathrm{ml}$ of $10 \mu \mathrm{g} / \mathrm{ml}$ human plasma FN was added to $10 \mathrm{~cm}$ culture dishes for $3 \mathrm{hrs}$ at $37^{\circ} \mathrm{C}$. The $\mathrm{FN}$ solution was removed and non-specific binding sites were blocked with $5 \mathrm{ml}$ phosphate buffered saline (PBS; $\mathrm{pH} 7.4$ ), $5 \%$ Bovine serum albumin (BSA). Harvested cells were suspended in the basic culture medium (RPMI for CHO cells, DMEM for RASM) supplemented with 1\% BSA. Mock and CD9-CHO (A6) cells or the transduced RASM cells (Ad-CD9 and Ad-LacZ) and untransduced control cells were plated onto FN coated culture dishes at $2.5 \times 10^{6}$ cells/dish and allowed to adhere for indicated time.

All the incubations were carried out in a humidified $5 \% \mathrm{CO}_{2}$ incubator at $37^{\circ} \mathrm{C}$. Control cells were held in suspension in RPMI or DMEM $+1 \%$ BSA at $37^{\circ} \mathrm{C}$. After adhesion, cells were washed two times in ice-cold PBS and lysed in Triton-X100 lysis buffer (Table 3.4) . Protein concentration in the samples was determined by Biorad ${ }^{D c}$ 
Table 3.3 Antibodies Used for the Detection of CD9- $\alpha 5 \beta 1$ Complex

\begin{tabular}{cccc}
\hline Antigen & Antibody for IP & $\begin{array}{c}\text { Primary Antibody } \\
\text { (Dilution) }\end{array}$ & $\begin{array}{c}\text { Secondary Antibody } \\
\text { (Dilution) }\end{array}$ \\
\hline$\alpha 5$ integrin & HM $\alpha 5-1$ & Anti- $\alpha 5, \mathrm{H}-104$ & DAR-HRP \\
& & $(1: 2,500)$ & $(1: 25,000)$ \\
$\beta 1$ integrin & HM $\beta 1-1$ & Anti- $\beta 1$, Clone- 18 & SAM-HRP \\
& & $(1: 1,000)$ & $(1: 25,000)$ \\
CDAb7 & Anti-CD9, C-4 & SAM-HRP \\
& & $(1: 500)$ & $(1: 50,000)$ \\
\hline
\end{tabular}


Table 3.4 Composition of Triton-X 100 Lysis Buffer

\begin{tabular}{ccc}
\hline Reagent & Stock & Final Concentration \\
Tris & $1 \mathrm{M}$ & $20 \mathrm{mM}$ \\
Deoxycholate & $3 \mathrm{M}$ & $150 \mathrm{mM}$ \\
SDS & $10 \%$ & $0.5 \%$ \\
Triton-X 100 & $10 \%$ & $0.1 \%$ \\
EDTA & $10 \%$ & $1 \%$ \\
Protease Inhibitor Cocktail & $100 \%$ & $5 \mathrm{mM}$ \\
Phosphatase Inhibitor Cocktail-I & $100 \%$ & $1 \%$ \\
Phosphatase Inhibitor Cocktail-II & $100 \%$ & $1 \%$ \\
\hline
\end{tabular}


protein assay kit and lysate equivalent to $40 \mu \mathrm{g}$ of protein was boiled with Laemmli buffer for $10 \mathrm{~min}$. The proteins were then resolved on SDS-PAGE and transferred onto Immobilon-P membrane as described above.

For Western blot analysis, membranes were first blocked for $6 \mathrm{hrs}$ at $4^{\circ} \mathrm{C}$ in TBST solution containing 5\% BSA. The blots were then incubated for $12-18 \mathrm{hrs}$ at $4^{\circ} \mathrm{C}$ in the primary antibody solution and the unbound antibody was removed by washing the blots with TBST. Subsequently, blots were incubated in horseradish peroxidase (HRP) conjugated donkey anti-rabbit (DAR) secondary antibody for an hr followed by washing the blots five times with TBST. Subsequently, the bands were developed with ECL substrate. As a loading control, the cut portions of the blots containing $40 \mathrm{kDa}$ actin bands were probed with mAb specific for actin (Chemicon, mAb 1501).

After analyzing the blots for phosphorylated Akt, the antibodies were removed with Restore Western blot stripping buffer. The membranes were incubated in Restore buffer for $15 \mathrm{~min}$ at $37^{\circ} \mathrm{C}$ with continuous agitation followed by manual rocking for 5 min. The blots were then washed with TBST and incubated in blocking buffer containing TBST and 5\% non fat dry milk. The membranes were then reprobed for total Akt with a polyclonal antibody (9272) against Akt. Table 3.5 shows the concentrations of various antibodies used in these experiments for Western blot analysis.

\subsection{Determination of Protein Tyrosine Phosphorylation}

Mock and A6 cells were seeded onto FN coated culture dishes as described above.

The cells were allowed to adhere for indicated time in a humidified 5\% $\mathrm{CO}_{2}$ incubator at $37^{\circ} \mathrm{C}$. Control cells were held in suspension in RPMI $+1 \% \mathrm{BSA}$ at $37^{\circ} \mathrm{C}$. Whole cell 
Table 3.5 Antibodies Used for the Western Blot Analysis of CHO Cell Lysates

\begin{tabular}{ccc}
\hline Antigen & $\begin{array}{c}\text { Primary Antibody } \\
\text { (Dilution) }\end{array}$ & $\begin{array}{c}\text { Secondary Antibody } \\
\text { (Dilution) }\end{array}$ \\
\hline p130Cas & Anti-p130Cas & SAM-HRP \\
& $(1: 1,000)$ & $(1: 25,00)$ \\
FAK & Anti-FAK & SAM-HRP \\
& $(1: 1,000)$ & $(1: 1,000)$ \\
Actin & Anti-CD9, C-4 & SAM-HRP \\
& $(1: 25,000)$ & $(1: 50,000)$ \\
Akt S473 & Phospho Akt (9271s) & DAR-HRP \\
& $(1: 1,000)$ & $(1: 25,000)$ \\
Akt & Total Akt (9272) & DAR-HRP \\
& $(1: 1,000)$ & $(1: 25,000)$ \\
Phospho Tyrosine & RC-20 HRP & - \\
& $(1: 2,500)$ & \\
\hline
\end{tabular}


lysates were prepared with Triton-X100 lysis buffer and protein concentration was estimated as described earlier. Lysate volume equivalent to $40 \mu \mathrm{g}$ for each sample was resolved on SDS-PAGE. For Western blot analysis, blots were blocked in TBST $+5 \%$ BSA and incubated with HRP conjugated anti-phosphotyrosine mAb (RC-20 HRP). Cell lysates were also analyzed for total levels p130Cas, focal adhesion kinase (FAK) and actin. Table 3.5 shows the concentrations of antibodies used in these experiments for Western blot analysis. The Western blots for FAK were developed with ECL regular substrate kit from Amersham Biosciences (Piscataway, NJ).

For determining p130Cas tyrosine phosphorylation, p130Cas was first immunoprecipitated from $100 \mu \mathrm{g}$ of lysates with $5 \mu \mathrm{l}$ of anti-p130Cas mAb (clone 21) described above. The immune complexes were captured with $75 \mu \mathrm{l}$ of protein $\mathrm{G}$ plus protein A beads and the captured proteins were resolved on a 7.5\% SDS-PAGE. For Western analysis, the blots were first probed for phosphorylated tyrosine with the RC-20 HRP antibody. For determining total p130Cas levels in the samples, the blots were stripped and reprobed with anti-p130Cas mAb.

\subsection{Transwell Migration Assays}

Haptotactic motility assays were performed using a modified Boyden chamber technique. For coating with FN, tissue culture inserts were placed into the wells of 24 well culture plates added with $200 \mu \mathrm{l}$ of $10 \mu \mathrm{g} / \mathrm{ml}$ human plasma FN solution and incubated for $3 \mathrm{hrs}$ at $37^{\circ} \mathrm{C}$. After incubation, excess FN solution was removed by blotting the edges of the inserts with absorbent paper. 
For the motility assays in the presence of pharmacological inhibitors, cell cycle synchronized Mock and A6 cells were seeded in to $75 \mathrm{~cm}^{2}$ flasks at a density of $2.5 \times 10^{6}$ cells/flask. All the motility experiments were performed within $18 \mathrm{hrs}$ after seeding the cells at this density. The inhibitor stock solutions were prepared by dissolving them in a solution of dimethyl sulfoxide (DMSO).

Cells were harvested by trypsinization for $2 \mathrm{~min}$, and the trypsin solution was neutralized and cells were suspended in RPMI-1640 medium containing 1\% BSA at a density of $3.1 \times 10^{5}$ cells $/ \mathrm{ml}$ and incubated for $30 \mathrm{~min}$ at $37^{\circ} \mathrm{C}$. Where inhibitors were used, $2 \mathrm{ml}$ suspension was removed and treated with inhibitor for $30 \mathrm{~min}$ at $37^{\circ} \mathrm{C}$. As a negative control, cells were similarly treated only with the DMSO alone. FN coated tissue culture inserts were placed in 24 well plates containing $300 \mu \mathrm{l}$ of RPMI+1\% BSA. Four hundred $\mu 1$ of cell suspension $\left(1.25 \times 10^{5} /\right.$ Insert $)$ was added to each insert.

Cells were allowed to migrate for $3 \mathrm{hrs}$ by incubating the culture plates at $37^{\circ} \mathrm{C}$ in humidified $\mathrm{CO}_{2}$ incubator. All the samples were tested in triplicate. After the motility assays, the cell suspension from the tissue culture inserts was aspirated and cells adhered to the undersurface of the Transwell chamber were stained by placing the inserts in 24 well plates containing $400 \mu \mathrm{l}$ of Wright-Giemsa stain for $10 \mathrm{~min}$. Four hundred $\mu \mathrm{l}$ of distilled water was then added to each well and staining was continued for another 10 $\min$.

The inserts were then air-dried for overnight. The membranes of tissue culture inserts were detached carefully, placed on glass slides, and fixed with Permount solution and cover-slips were added. Cells from five random fields were counted under 100X 
magnification. In each experiment, for each sample, mean cell numbers from three inserts were compared between different treatment groups.

To examine the effect of serum on cell migration, Mock and A6 cells were initially maintained in RPMI medium containing $1 \%$ serum for $18 \mathrm{hrs}$ prior to the assay. Subsequent steps were carried out as described above. For motility experiments performed with the $\alpha 5 \beta 1$ blocking antibody (PB1), Mock and A6 cells were treated with indicated concentration of $\mathrm{PB} 1$ for $30 \mathrm{~min}$ at $37^{\circ} \mathrm{C}$. As a negative control for this experiment, an isotype matched antibody (MOPC-21) was used to pretreat the cells. For the migration experiments performed after transient transfections, cells were harvested after an indicated period after transfection and subjected to haptotactic motility assays as described above.

\subsection{Transient Transfection with Dominant/Negative PI-3K}

The $\Delta$ p85 plasmid construct, containing cDNA corresponding to dominant/negative p85 subunit ( $\Delta \mathrm{p} 85)$ of PI-3K has been described previously $(217)$. The pEGFP-N1 plasmid capable of expressing a red shift variant of GFP was obtained from BD Biosciences. Transient transfections of Mock and A6 cells were done using lipofection. Optimization experiments were performed to determine the ratios of plasmid: lipofectamine reagent required for maximal transfection efficiency and these conditions were used throughout these studies.

Twenty four hrs prior to transfection, the growth media was replaced with RPMI1640 containing $10 \%$ FBS (without Geneticin) and cells were transfected with $25 \mu \mathrm{g}$ of plasmid DNA mixed with $25 \mu 1$ of Plus reagent and $75 \mu$ l of Lipofectamine in $6 \mathrm{ml}$ of 
Optimem and cells were incubated with lipid-DNA complexes for $12 \mathrm{hrs}$. Cells transfected with pEGFP-N1 plasmid alone were used as negative controls. The transfection medium was removed and $10 \mathrm{ml}$ of RPMI growth medium was added. Thirty-six hrs after transfection, cells were harvested for lysate preparation and haptotactic motility experiments. Prior to motility assays the viability of cells was assessed by trypan blue dye exclusion.

\subsection{1 p130Cas siRNA Transfection of CHO Cells}

All the reagents required for p130Cas siRNA transfection of cells were purchased from Santa-Cruz Biotechnology. As a negative control, non-specific siRNA duplexes were used in the transfection experiments. Lyophilized siRNA duplexes were reconstituted in the siRNA dilution buffer and the stock solutions were stored at $-80^{\circ} \mathrm{C}$ for subsequent use. Cultured Mock and A6 cells were transfected with p130Cas siRNA duplex as follows.

Cultured Mock, A6 cells were harvested by trypsinization and were seeded in $6 \mathrm{~cm}$ polystyrene tissue culture dishes at a density of $6 \times 10^{5}$ cells / dish. For each transfection, the following solutions were prepared- solution-A was prepared by adding $24 \mu 1$ of $20 \mu \mathrm{M}$ stock solution of p130Cas siRNA duplex or the control siRNA duplex to $300 \mu \mathrm{l}$ of siRNA transfection medium and solution-B was prepared by adding $24 \mu \mathrm{l}$ of siRNA transfection reagent to $300 \mu \mathrm{l}$ of siRNA transfection medium. Solution-A and solution-B were then mixed and incubated for $30 \mathrm{~min}$ at room temperature. Final transfection medium was prepared by adding the mixture of solution-A and B to $2.4 \mathrm{ml}$ for transfection medium. 
At the time of transfection, cells were washed twice with $2.5 \mathrm{ml}$ of RPMI followed by $2.5 \mathrm{ml}$ of siRNA transfection medium. The final transfection mixture was added dropwise to the culture dishes with manual agitation. The transfected cells were then incubated for $6 \mathrm{hrs}$ in humidified $5 \% \mathrm{CO}_{2}$ incubator at $37^{\circ} \mathrm{C}$ when the transfection medium was removed and $5 \mathrm{ml}$ of growth medium was added and plates returned to the incubator. At 24 hrs post transfection siRNA transfected cells were either lysed for Western blot analysis, or harvested for haptotactic motility assays.

\subsection{Data Analysis}

For all the motility experiments, mean cell numbers from at least three independent experiments were calculated and statistical significance was tested by oneway analysis of variance (ANOVA, Boneferroni test) using Sigma Stat software package. Results were considered statistically significant if a $p$ value of $<0.05$ was reached. For quantitation of Western blot data, the following method was used. All the blots were first scanned and the TIFF files were exported to NIH Image software for subsequent analysis. For each band, representative integrated density values were computed after subtracting the background density. Mean integrated densities values from three independent experiments were calculated and their ratios with control bands were calculated using Microsoft EXCEL programming. 


\section{Chapter 4: Results and Discussion}

\subsection{Signal Transduction Mechanisms of CD9 Mediated Cell Migration}

Cell migration is a complex phenomenon that forms the basis for several important biological processes such as embryonic development (218), immunity (219) and wound healing (220). Despite its complex nature, cell migration in organisms is tightly regulated and abnormal cellular migration contributes to spread of malignant cells from their primary site to the distant organs (tumor metastasis) (221). In addition to this, abnormal migration of smooth muscle cells in the vascular wall is an important contributing factor for the pathogenesis of vascular disorders such as atherosclerosis and restenosis. Cells respond to the environmental cues such as chemoattractants and growth factors (222) and extracellular matrix (ECM) proteins (223) via specific cell surface receptors. Activation of these cell surface receptors in turn leads to initiation of signaling pathways that ultimately affect cytoskeletal dynamics and cell migration (224).

Integrins are heterodimeric integral membrane proteins that act as receptors to ECM proteins $(24,25)$. Integrin interactions with the ECM proteins are critical for maintaining the specific adhesive phenotypes of the cells. Like integrins, tetraspanins have also been implicated in the modulation of adhesion dependent phenomenon such as cell migration (137). Several lines of evidence suggest that CD9 is important in the regulating cell migration. CD9 expression has been shown to correlate with tumor metastasis $(28,115,118,119,225-228)$ and studies using the monoclonal antibodies specific for CD9 have shown that CD9 modulates cell adhesion and migration on to ECM proteins $(91,95,96,121-124,127)$. 
Our earlier studies demonstrate that expression of human CD9 in CHO cells significantly influences haptotactic cell migration to the ECM ligand FN (129). Mechanistically, haptotactic cell migration involves directional migration of cells to the immobilized ECM proteins. Using modified Boyden chambers to perform motility experiments, it has been shown that expression of human CD9 in CHO cells leads to 3-5 fold increase in haptotactic motility on to FN (129). A peptide corresponding to the CD9 EC2 region (peptide 6; EC2 168-192) was shown to significantly inhibit CD9 promoted haptotactic motility to FN (129). Furthermore, analysis of CHO cells expressing CD9 deletion mutants showed that $\mathrm{EC} 2$ region of $\mathrm{CD} 9$ is required for this phenotype (129). Intracellular signaling mechanisms governing CD9 mediated events are poorly understood. Above and beyond serving as molecular organizers, tetraspanins have been implicated in the transduction of adhesion dependent signals across the membrane. Tetraspanins CD9, CD63, CD151, and CD81 associate with PI-4K (20). Another tetraspanin CD82 has been shown to associate with specific isoforms of PKC ( $\alpha$ and $\beta \mathrm{II}$ ) and this association was shown to be independent of CD82-integrin association (38). Although CD9 was shown to activate multiple signaling pathways, the signaling mechanisms by which CD9 regulates cell motility are not known.

In an effort to understand the molecular basis of CD9 mediated motility, we investigated the role of intracellular signaling molecules required for CD9 promoted haptotactic cell motility. To address these questions, small molecule inhibitors for specific signaling pathways were used in the haptotactic motility assays to initially screen and identify the signaling pathway responsible for CD9 promoted haptotactic cell migration. 
The intracellular signaling pathways, PKC (202) MAPK (203), and PI-3K (229) have been previously reported to be involved adhesion molecule mediated signaling pathways. Pharmacological inhibitors for all these pathways are commercially available and are routinely being used to test the role of these pathways. Table 4.1 lists the pharmacological inhibitors that were used in motility experiments and the pathway/s they are intended to inhibit.

The PKC is a large family of protein kinases that includes several isoforms. Depending on the requirement of a specific activating factor, the PKC family is further divided in to 3 subfamilies: classical or conventional, novel and atypical (230-232). The classical PKCs include $\alpha, \beta_{\mathrm{I}}, \beta_{\mathrm{II}}, \gamma$ isoforms that are activated by diacylglyerol (DAG) and phosphatidylcholine that are generated downstream of phospholipase $\mathrm{C}$ signaling (233-235).

Classical PKCs have been shown to play an important role in integrin mediated intracellular signaling pathways (236-238). Specifically, studies have shown that PKC $\alpha$ regulates $\beta 1$-integrin dependent cell motility (202). Biochemical evidence also suggests that TM4SF proteins including CD9 exist in complex with the activated form of classical PKCs (38). CD9 has been shown to exist in complex with $\beta 1$ integrin in CHO cells (200). Therefore, we investigated the possible role of classical PKCs in CD9 mediated haptotactic motility.

Gö 6976 compound was shown to selectively inhibit classical PKCs $\alpha$ and $\beta$ (239). Nanomolar (nM) concentrations of Gö 6976 were shown to inhibit the activity of Ca2+-dependent isozymes of PKC in a highly specific manner (239). To study the contribution of PKC, PKC activity in Mock and A6 cells was inhibited with the Gö 6976 
Table 4.1 Inhibitors Used in CHO Cell Haptotactic Motility Experiments

\begin{tabular}{ccc}
\hline Serial No & Compound & Pathway Inhibited \\
\hline 1 & Gö 6976 & Protein Kinase C (PKC) \\
2 & PD98059 & Mitogen Activated Protein Kinase (MAPK) \\
3 & Wortmannin & Phosphatidylinositol-3 Kinase (PI-3K) \\
4 & LY294002 & Phosphatidylinositol-3 Kinase (PI-3K) \\
\hline
\end{tabular}


compound and haptotactic motility assays were performed. Motility of Mock and A6 cells were tested at $50 \mathrm{nM}$ and $100 \mathrm{nM}$ of Gö 6976. As shown in Figure 4.1, treatment of Mock and A6 cells with DMSO alone did not significantly affect haptotactic motility of cells. Motility of Mock and A6 cells treated with two different concentrations (50 nM and $100 \mathrm{nM}$ ) of Gö 6976 were also not significantly different from respective DMSO treated controls. Inhibition of CD9 promoted cell migration was not observed at Gö 6976 concentrations as high as $14 \mu \mathrm{M}$ (data not shown). The results suggest that suppression of endogenous classical PKC activity is not sufficient to inhibit CD9 promoted cell migration in $\mathrm{CHO}$ cells.

Cell interaction with the ECM ligands activates an important intracellular signaling pathway known as the MAPK $(203,240)$. The mechanism of activation of MAPK involves the following steps. The first in the MAPK activation requires activation of receptor tyrosine kinases (RTKs) by ligand induced autophosphorylation (241). Phosphorylated RTKs are then recognized by src homology domain-2 (SH-2) containing adapter proteins such as Grb2 and Shc. Adapter proteins Grb2 and Shc recruit proteins like SOS subsequently leading to activation of Ras. Activated Ras further recruits and activates Raf-1 which directly phosphorylates and activates MEK1/MEK2. Activated MEK1/MEK2 phosphorylate and activates the extracellular signal regulated kinases (ERKs) (203).

Integrins were reported to activate MAPK pathway by several mechanisms. Integrins modulate growth factor receptors (RTKs) activity thereby directly activating MAPK $(242,243)$. Integrin mediated activation of FAK and its phosphorylation on tyrosine (Y) 925 can also activate the MAPK pathway (244). Integrins were also reported 


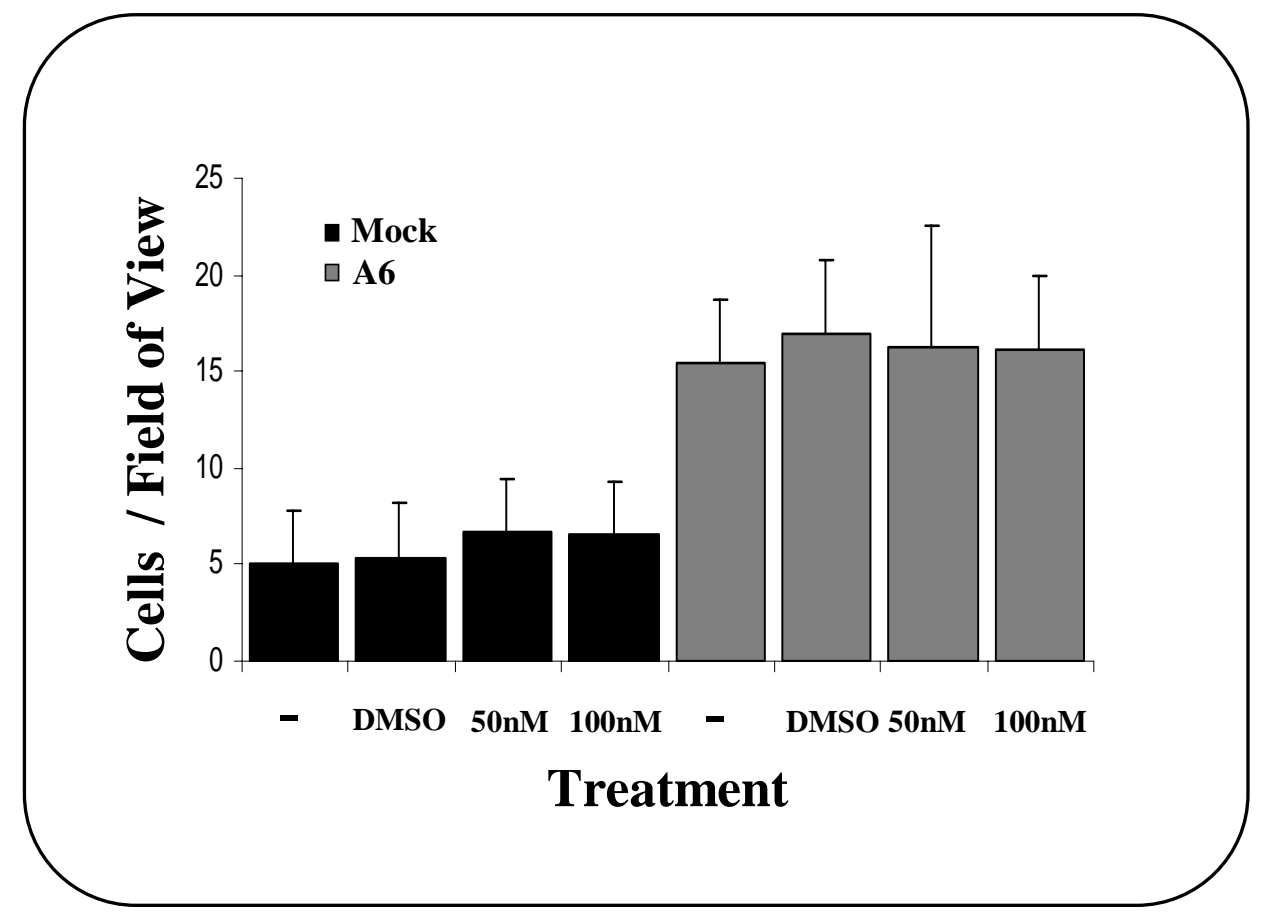

Figure 4.1 Effects of PKC Inhibitor Gö 6976 on Cell Motility

Mean cell migration of Mock and A6 cells $(n=2)$ treated with DMSO, $50 \mathrm{nM}$ and $100 \mathrm{nM}$ of PKC inhibitor Gö 6976. The error bars represent the standard deviation. 
to activate the intermediate molecules of the MAPK pathway such as Raf-1 and MEK1 $(245,246)$. Although the exact mechanisms are not understood both anti-CD9 mAb treatment and ectopic expression of CD9 were shown to regulate MAPK pathway in certain cell types $(188,190)$.

Therefore, we examined whether CD9 mediated haptotactic cell motility requires MAPK pathway. Since, MEK is an upstream activator of ERK, inhibition of MEK activity leads to suppression of the MAP kinase pathway. PD98059 is a cell permeable molecule that selectively inhibits MEK1 activity thereby inhibiting MAPK pathway (247, 248). Reported inhibitory concentration 50 (IC50) of PD98059 for different MAPK activators were reported to be in the micromolar $(\mu \mathrm{M})$ range (247). To address the importance of MAPK pathway in CD9 induced motility, we performed motility assays at $50 \mu \mathrm{M}$ and $100 \mu \mathrm{M}$ PD98059. At both the concentrations tested, CD9 enhanced motility was not significantly affected as compared to the respective DMSO treated controls (Figure 4.2). These results suggested that MAP kinase pathway may not be crucial for the CD9 mediated haptotaxis.

Activation of integrins also leads to initiation of signaling through intracellular PI-3K (204). PI-3K is a large family of lipid kinases capable of phosphorylating the $3^{\text {rd }}$ hydroxyl group of the inositol ring of phosphatidylinositol (PI) (249). Depending upon the substrate specificity, PI-3K family is subdivided into 3 classes: Class I, II, and III. Class I PI-3K, phosphorylates PI 4, 5 bisphosphate to generate PI 3, 4, 5 triphosphate. Structurally, Class I PI-3K enzymes are heterodimeric proteins comprising of a regulatory subunit (p85) and a catalytic subunit (p110) (250). PI-3K is activated upon binding of Src homology-2 (SH2) domain of the regulatory subunit (p85) binds the 


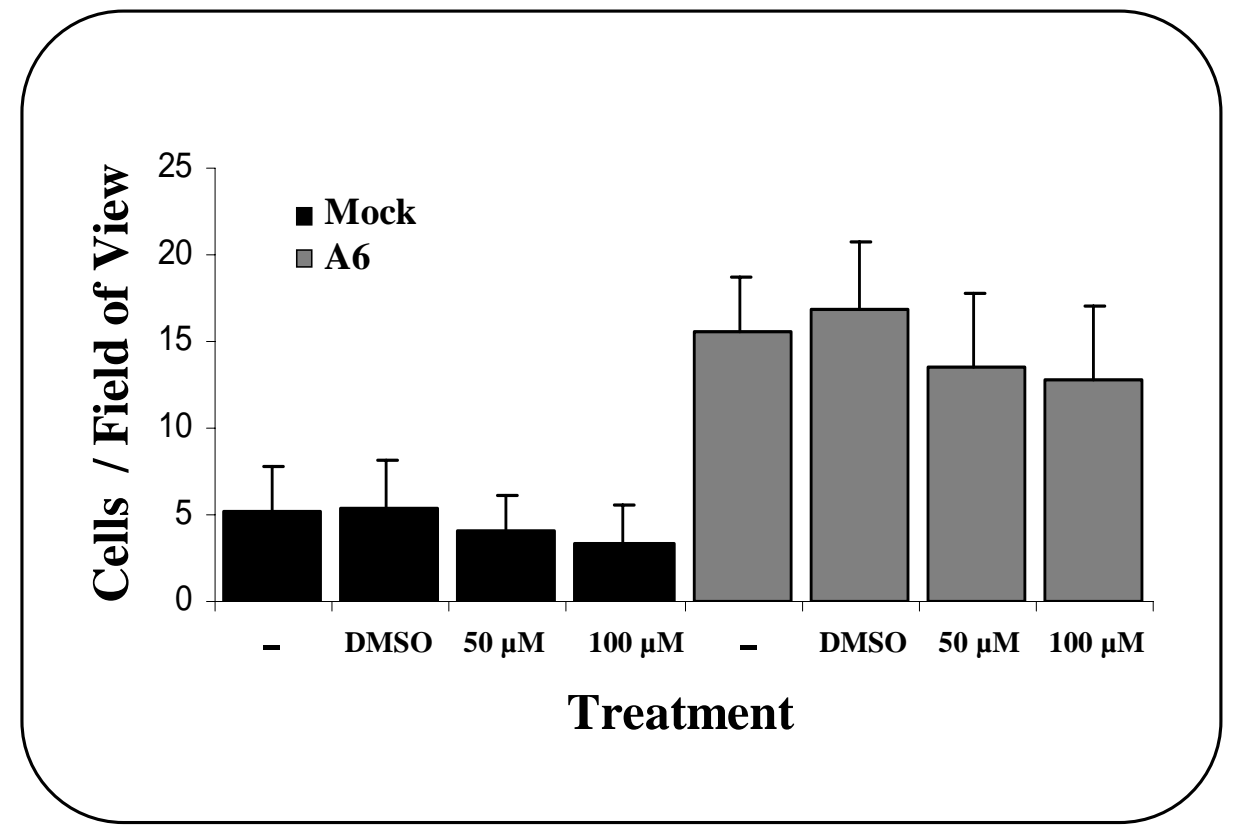

Figure 4.2 Effects of MAPK Inhibitor PD98059 on Cell Motility

Mean cell migration of Mock and A6 cells $(n=2)$ treated with DMSO, 50 and $100 \mu \mathrm{M}$ concentration of MAPK inhibitor, PD98059. The error bars represent the standard deviation. 
phosphotyrosine residues (251) generated upon activation of growth factors or integrins.

The p85 subunit of PI-3K then recruits the catalytic (p110) subunit into the proximity of membrane associated phosphoinositides. The p110 catalyses the phosphorylation of $3^{\text {rd }}$ hydroxyl group of the inositol ring leading to generation of phosphatidylinositol-3 phosphates. It has widely been accepted that PI-3K pathway is important for cell migration. For example, in $\mathrm{CHO}$ cells, haptotactic motility to FN was shown to be predominately regulated by the PI-3K pathway (252). Based on these observations, we examined the contribution of PI-3K to CD9 mediated haptotactic cell migration.

In independent experiments, two inhibitors of PI-3K pathway were used in the haptotactic motility assays. Wortmannin, is a fungal metabolite that acts by irreversiblyinhibiting the catalytic subunit of the PI-3K (253). Wortmannin, while at nM concentrations specifically inhibits $\mathrm{PI}-3 \mathrm{~K}, \mu \mathrm{M}$ concentrations were shown to inhibit other important signaling molecules such as the myosin light chain kinase and phospholipase D that are involved in cell migration $(254,255)$. Therefore the effects of wortmannin on Mock, A6 cell migration were tested at three different concentrations.

Figure 4.3 depicts the results of the Mock, A6 cell migration in the presence of wortmannin. Inhibition of A6 cell migration was observed at wortmannin concentrations as low as $0.2 \mu \mathrm{M}$ ( $\mathrm{p}=0.007$ as compared to DMSO control) while higher concentrations of wortmannin $(10 \mu \mathrm{M})$ inhibited the migration of both Mock and A6 cells. Thus wortmannin had a dose dependent inhibition of A6 cell migration while vehicle control DMSO did not affect the motility of Mock or A6 cells. 


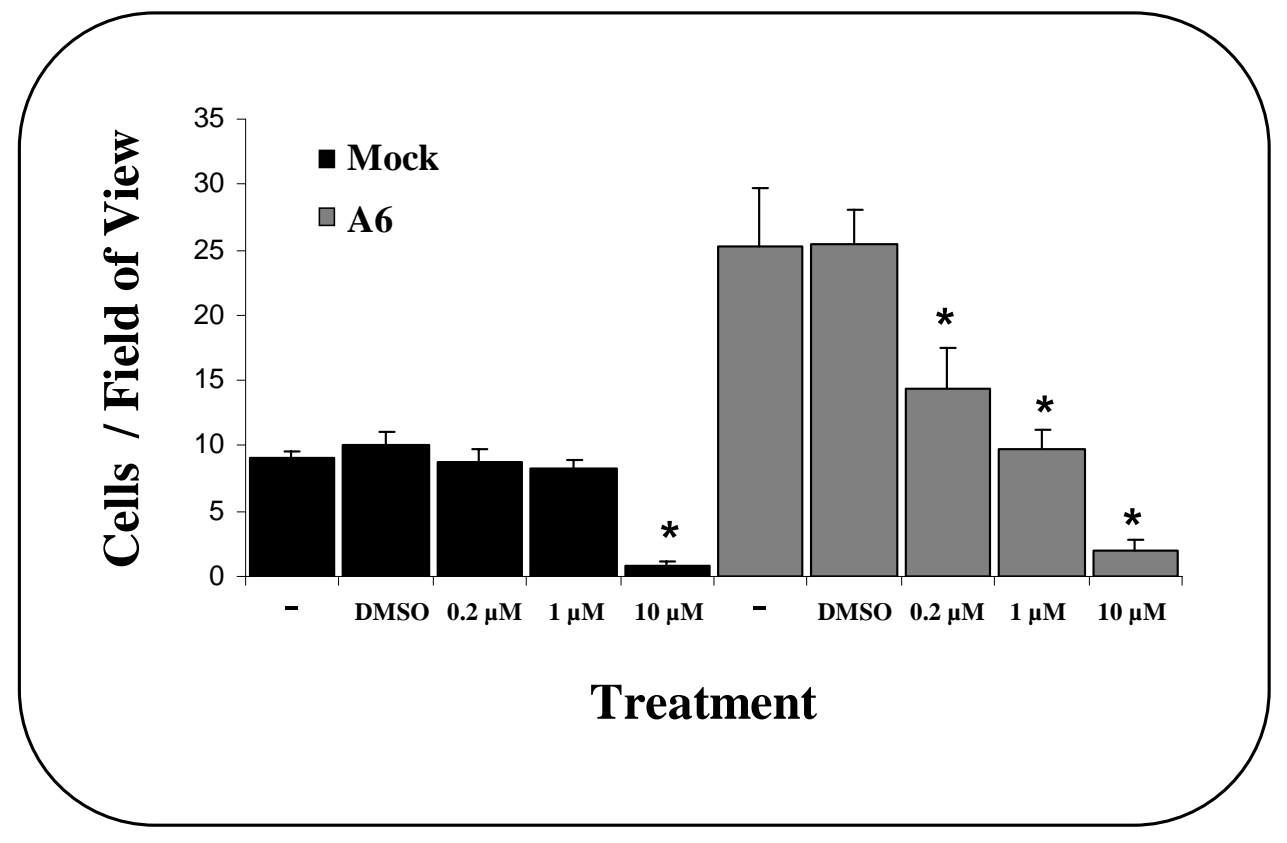

Figure 4.3 Effects of PI-3K Inhibitor Wortmannin on Cell Motility

Mean cell migration of Mock and A6 cells $(n=3)$ treated with DMSO and $0.2,1,10 \mu \mathrm{M}$ wortmannin. * Indicates p value $<0.05$ as compared to the respective DMSO (0) treated control group. The error bars represent the standard deviation. 
LY294002 is a specific inhibitor of PI-3K that competes with ATP at the catalytic site of the enzyme (256). LY294002 has been shown to be a more selective inhibitor of PI-3K than wortmannin. Therefore, to validate the results obtained with wortmannin, motility assays were performed with LY294002. Figure 4.4 shows the results of the motility assays performed in the presence of two different concentration of LY294002. The results indicate that inhibition of PI-3K by LY294002 had a dose response inhibition on CD9 promoted haptotactic cell migration. A statistically significant difference in A6 cell migration was observed with LY294002 concentration of 25 and $50 \mu \mathrm{M}(\mathrm{p}<0.001)$ as compared to DMSO control). Carrier compound DMSO did not significantly affect the motility of Mock or A6 cells.

In summary, motility assays performed with pharmacological inhibitors to crucial signaling molecules clearly indicated that CD9 promoted haptotactic cell migration in $\mathrm{CHO}$ cells does not require either PKC or MAPK. The finding that CD9 promoted migration is blocked by two structurally different inhibitors of the PI-3K pathway suggest that PI-3K may be an essential pathway required for $\mathrm{CD} 9$ mediated cell migration.

\subsection{PI-3K in CD9 Mediated Cell Motility}

In order to confirm the results obtained with the pharmacological inhibitors of PI$3 \mathrm{~K}$, we aimed to suppress the endogenous PI-3K activity by molecular methodology. Dominant/negative forms of the protein are widely being used to suppress the activity of several signaling molecules (257). Since dominant/negative forms exclusively down regulate the function of targeted protein of interest, they are in a way more specific than pharmacological inhibitors to inhibit a specific signaling pathway. 


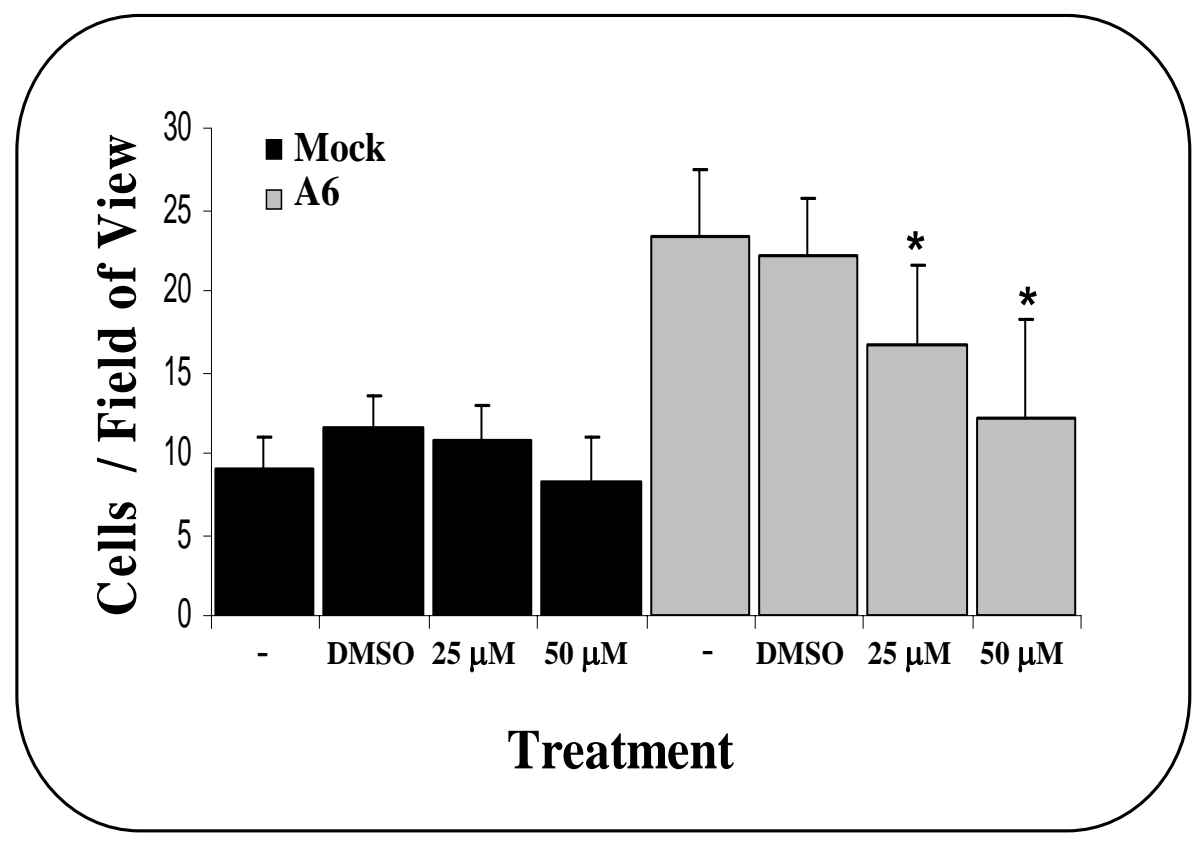

Figure 4.4 Effects of PI-3K Inhibitor LY294002 on Cell Motility

Mean cell migration of Mock and A6 cells $(n=3)$ treated with DMSO and 25 and $50 \mu \mathrm{M}$ LY294002. * Indicates p value $<0.05$ as compared to the DMSO treated A6 cells. The error bars represent the standard deviation. 
Suppression of PI-3K activity in cells can be achieved by overexpression of dominant/negative form of the PI-3K. The PI-3K dominant/negative construct used in our study expresses a mutant form of the regulatory subunit designated as $\Delta \mathrm{p} 85$. The $\Delta \mathrm{p} 85$ form of PI-3K lacks the crucial domain that is necessary for interaction with the p110 subunit. Since, p110 subunit interaction with the regulatory (p85) subunit is a prerequisite for PI-3K activation, over expression of $\Delta \mathrm{p} 85$ leads to suppression of endogenous PI-3K activity (217).

Mock and A6 cells were transiently transfected either with a GFP expression plasmid (pEGFP-N1) or a plasmid that expresses a $\Delta \mathrm{p} 85$ as described in Materials and Methods. The conditions for the expression of the $\Delta \mathrm{p} 85$ subunit were optimized using the lipofectamine protocol. In all the experiments, expression of $\Delta \mathrm{p} 85$ subunit was confirmed by Western blot analysis of whole cell lysates made $72 \mathrm{hrs}$ post transfection. A polyclonal antibody against p85 subunit was employed in the western blots.

As shown in Figure 4.5, both Mock and A6 cells expressed comparable levels of endogenous p85 subunit and transient expression of $\Delta \mathrm{p} 85$ plasmid but not the GFP plasmid led to expression of $\Delta \mathrm{p} 85$ subunit. As expected, the endogenous $\mathrm{p} 85$ was recognized as a separate band that ran at slightly higher molecular weight than the overexpressed $\Delta \mathrm{p} 85$ (Figure 4.5).

Since PI-3K pathway is important in cell survival and proliferation pathways, over expression of dominant/negative form of PI-3K has been shown to induce apoptotic cell death (258). Since we intended to use the dominant/negative PI-3 kinase transfected $\mathrm{CHO}$ cells in migration assays; viability of cells was first assessed by trypan blue dye exclusion before subjecting the cells to haptotactic motility. Consistent with an earlier 


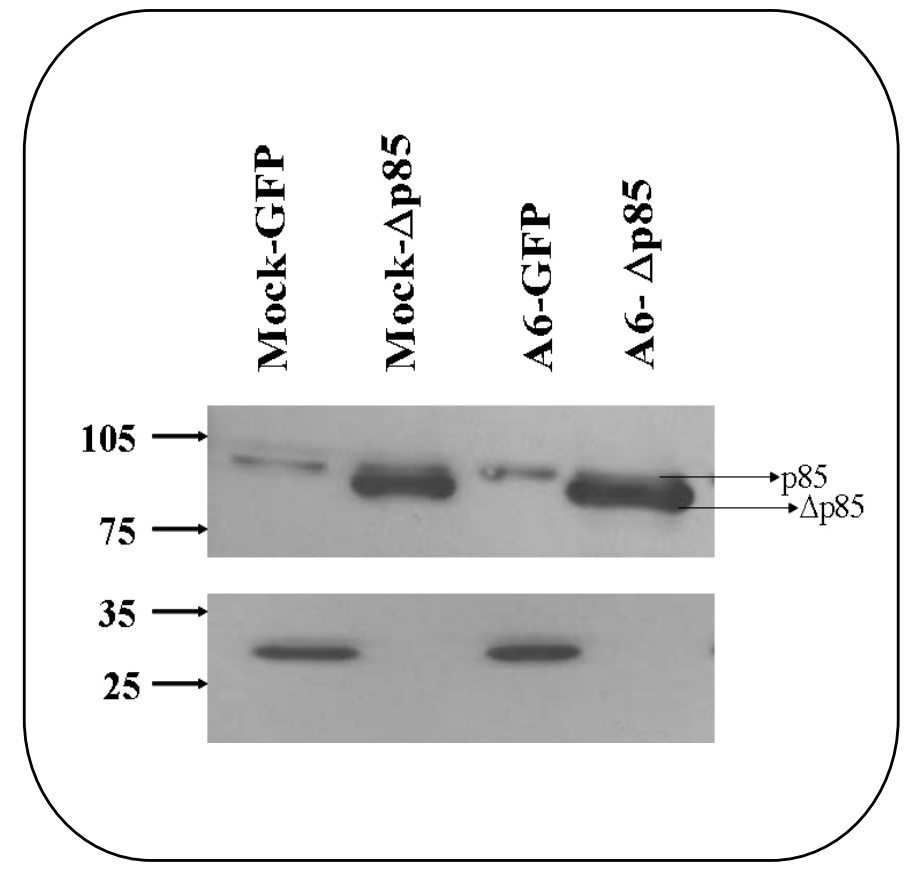

Figure 4.5 Western Blot Analysis after Dominant/Negative PI-3K Transfection

Expression of $\Delta \mathrm{p} 85$ and GFP in Mock and A6 cells after transient transfection with $\Delta \mathrm{p} 85$ and GFP expression plasmids. Endogenous PI-3K (p85) and the $\Delta \mathrm{p} 85$ (top panel) were detected with a polyclonal anti-PI-3K antibody and GFP (bottom panel) was detected with an anti-GFP mAb. 
finding (259), expression of dominant/negative PI-3K plasmid in CHO cells did not affect the cell viability.

To test the effects of dominant/negative PI-3K in CD9 mediated haptotactic cell motility, Mock and A6 cells transiently transfected with pEGFP-N1 or the $\Delta \mathrm{p} 85$ plasmid were subjected to transwell migration assays on FN. As shown in Figure 4.6, dominant/negative transfections with the $\Delta \mathrm{p} 85$ construct significantly inhibited migration of A6 cells ( $\mathrm{p}=0.039)$ when compared to the Mock cells. The expression of GFP plasmid

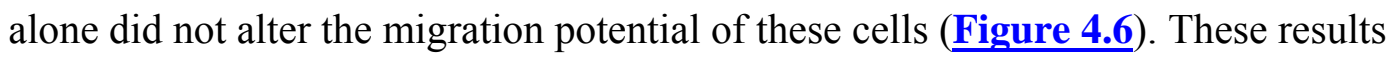
clearly show that PI-3K pathway is crucial for CD9 mediated cell migration on FN.

Subsequent to PI-3K activation, phosphoinositides are specifically recognized by the pleckstrin homology $(\mathrm{PH})$ domain containing proteins. Akt, also known as protein kinase $\mathrm{B}(\mathrm{PKB})$, is a $\mathrm{PH}$ domain containing serine/ threonine protein kinase that is phosphorylated and activated downstream of PI-3K (260). Akt is specifically phosphorylated on a serine 473 residue in a PI-3K dependent manner $(261,262)$.

Phosphorylation of Akt on serine 473 has been shown to correlate with the kinase activity (261). Thus Akt phosphorylation on serine 473 residue serves as an indicator of PI-3K activity.

As our results using pharmacological inhibitors and the dominant/negative PI-3K showed the involvement of PI-3K in CD9 mediated cell motility, we next compared the phosphorylation state of Akt in Mock and A6 cells in response to adhesion to FN. For these experiments, cultured Mock and A6 cells were harvested and whole cell lysates were prepared from cells either held in suspension or that were plated onto FN for $1 \mathrm{hr}$. Subsequently, Akt phosphorylation was examined using a specific antibody that 


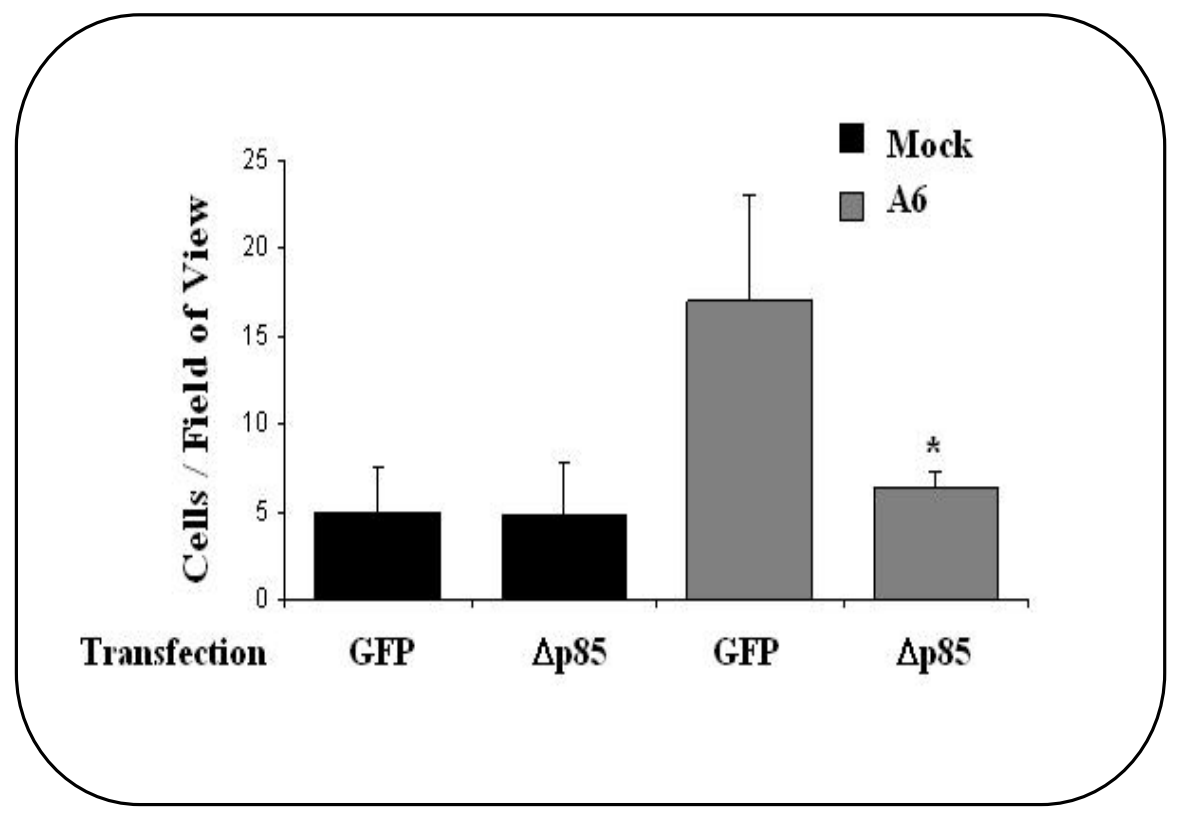

Figure 4.6 Effects of PI-3K Dominant/Negative $(\Delta \mathrm{p} 85)$ Transfection on Cell Motility Mean cell migration of Mock and A6 cells $(n=3)$ treated with control GFP plasmid and $\Delta \mathrm{p} 85$ plasmid. * represents $\mathrm{p}<0.05$ as compared to the GFP transfected A6 cells. The error bars represent the standard deviation. 
specifically binds phosphorylated S473 in Western blots.

Cell adhesion to FN can activate PI-3K in integrin $\alpha 5 \beta 1$ dependent manner. Therefore, as expected in response to cell adhesion to FN, Akt phosphorylation was increased both in Mock and A6 cells when cells were plated on to FN as compared to the suspension cells ( $\underline{\text { Figure 4.7) }}$. Consistent with our earlier observations that CD9 can stimulate PI-3K pathway, CD9 expressing cells had significantly higher levels of phosphorylated Akt when cells were plated onto FN compared to Mock controls (Figure 4.7).

No significant differences in Akt phosphorylation were observed between Mock and A6 cells when cells were held in suspension. As the differences in phosphorylation states can be attributable to the total amount of protein present in the samples total cellular levels of actin were also compared between Mock and A6 cells. As shown in bottom panel of Figure 4.7, total levels of cellular actin did not significantly differ for Mock and A6 cells. To quantify the data, scanned Western blots were subjected to densitometry scanning and phosphorylation of Akt was expressed in relation to actin. As shown in Figure 4.8, one hr after plating the cells on to FN, A6 cells have a three fold increase in relative Akt phosphorylation as compared to Mock cells.

\subsection{Adenoviral Vectors for Transient Expression of CD9}

Adenovirus mediated transient expression of transgenes has significant advantage over generation of stable cell lines expressing the gene of interest for the following reasons. Firstly, adenoviral system can be used to express proteins in cells that have limited in vitro growth capabilities (primary cells) and where generating stable cell line is 


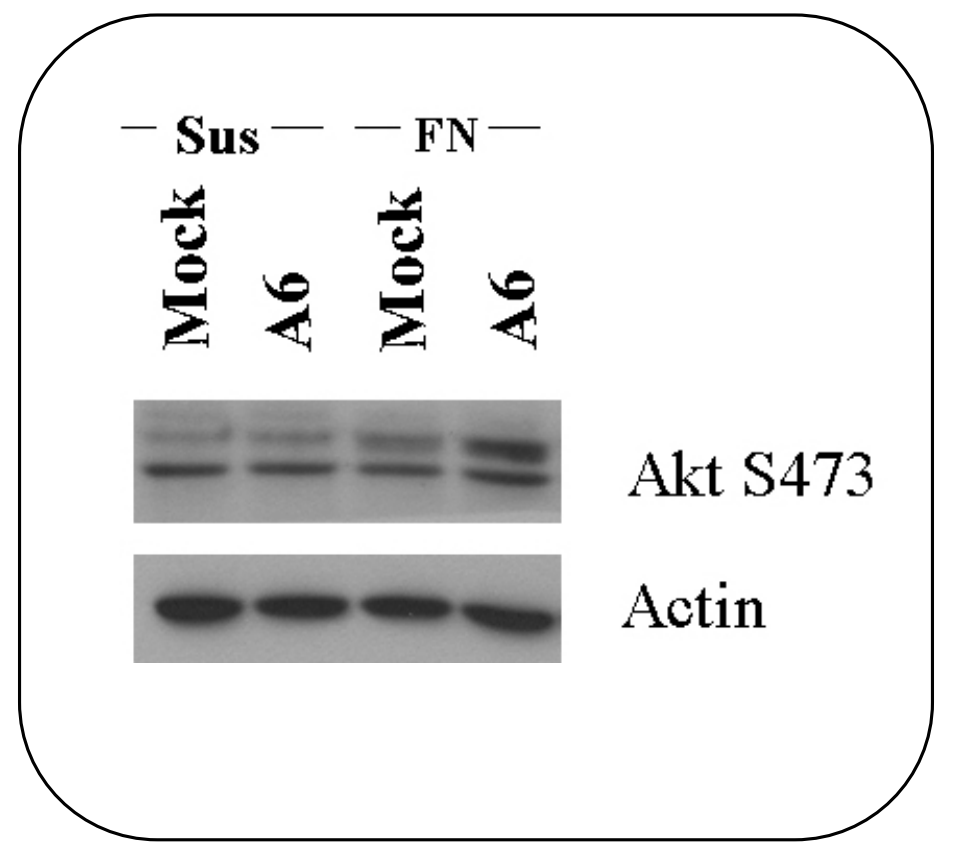

Figure 4.7 Effects of Stable Expression of CD9 on Akt Phosphorylation Mock and A6 cells held in suspension (sus) or plated on to FN for $1 \mathrm{hr}$ were analyzed for the phosphorylation of Akt on Serine 473 with a phospho-specific antibody (top panel). The same lysates were analyzed for total cellular actin (bottom panel). 


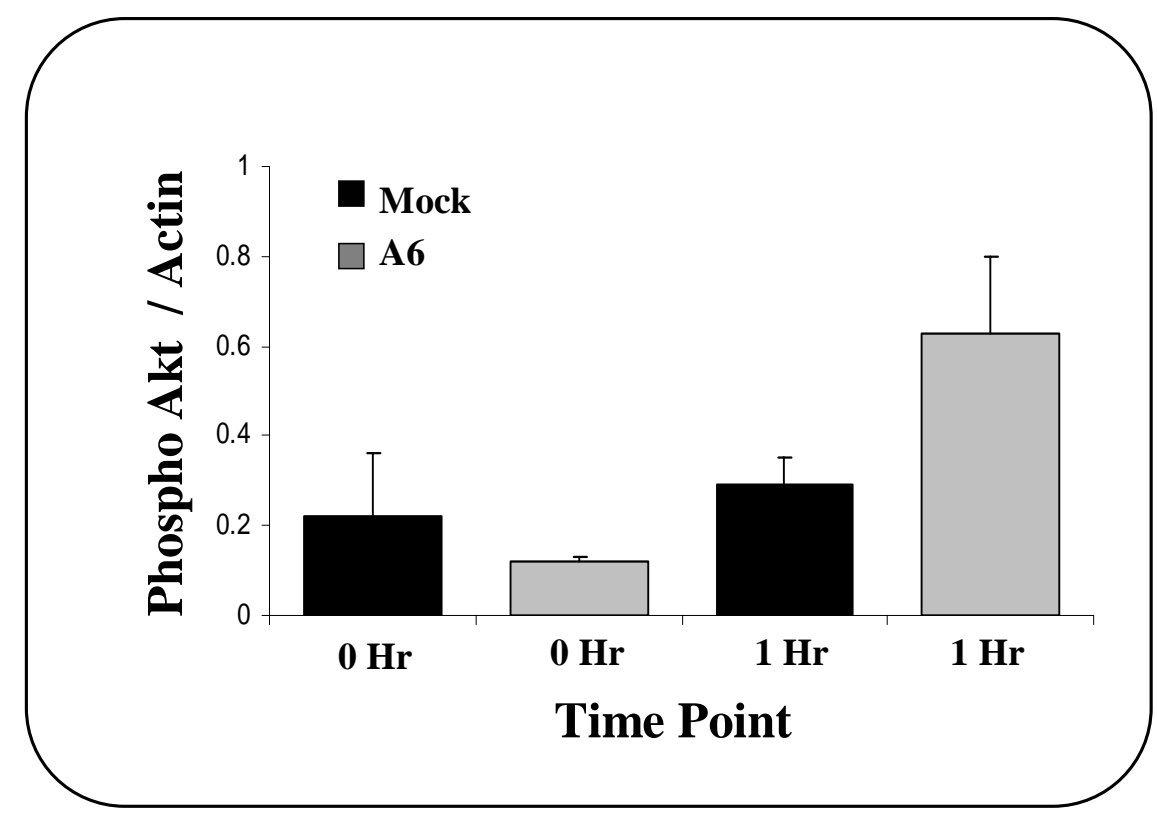

Figure 4.8 Quantification of Akt Phosphorylation in Mock and A6 Cells

Ratios of phosphorylated Akt and actin were compared between Mock and A6 cells either held in suspension $(0 \mathrm{hr})$ or plated onto FN $(1 \mathrm{hr})(\mathrm{n}=3)$. The error bars represent the standard deviation. 
not possible. Secondly, the levels of expression of the protein of interest can be controlled by using different virus multiplicity of infection (MOI). Thirdly, adenovirus does not integrate into the host genome thus avoiding the problems associated with the random insertions of transgenes into the native gene sequences. Finally, adenoviral vectors can be used for in vivo gene transfer in to animal tissues. For these reasons, adenovirus capable of expressing full-length human CD9 was generated as described in the Materials and Methods section.

At first, we tested the ability of adenovirus to express full-length CD9 (Ad-CD9) in cultured cells. Expression of CD9 was evaluated $72 \mathrm{hrs}$ after the adenoviral transduction. Figure 4.9 depicts the Western blot results of CD9 expression in primary rat aortic smooth muscle (RASM) cells transduced with three different concentrations of the Ad-CD9 virus. As expected, the levels of CD9 expression correlated with viral dose and maximal expression of CD9 was seen at 100 MOI. Despite the extent of CD9 expression, adenoviral transductions did not affect the viability of cells as determined by trypan blue exclusion. Therefore, an MOI of 100 was chosen for subsequent experiments.

In order to corroborate the Western blot results and to confirm successful expression of human CD9 as a surface protein in RASM cells, FCM was used. FCM experiments with untreated and Ad-CD9 treated RASM cells showed that the anti-human $\mathrm{CD} 9 \mathrm{mAb}(\mathrm{mAb} 7)$ caused a significant right shift in the mean fluorescence intensity only in the Ad-CD9 treated cells (igure 4.10). The isotype control antibody (MIgG, MOPC21) only showed background fluorescence in both untreated and Ad-CD9 treated cells (Figure 4.10). These results clearly showed that after Ad-CD9 treatment, human CD9 was successfully expressed on the cell surface of RASM cells. Similar results were 


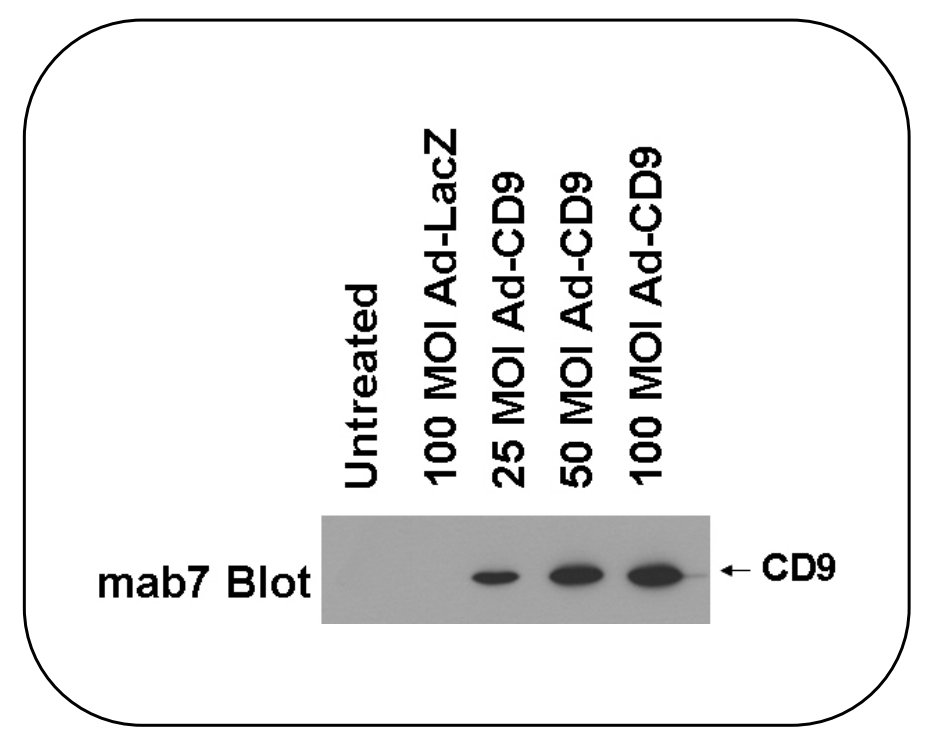

Figure 4.9 Western Blot Analysis for CD9 Expression after Adenoviral Transduction Untreated, Ad-LacZ treated, and Ad-CD9 treated (25, 50,100 MOI) vascular smooth muscle cells were analyzed for CD9 expression using anti-human CD9 (mAb7). MOI: multiplicity of infection. 


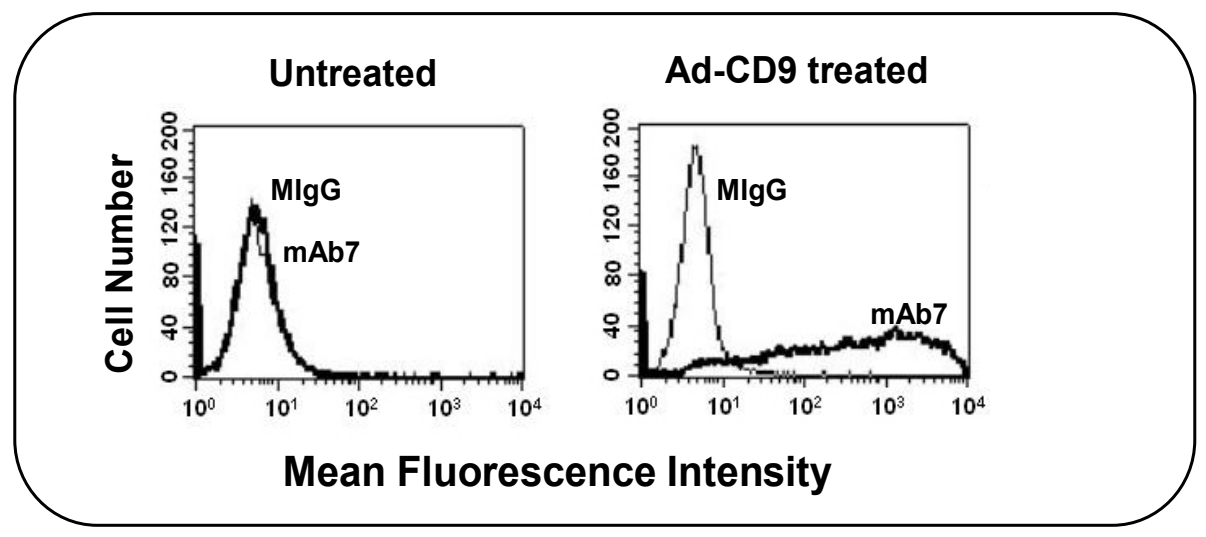

Figure 4.10 FCM Analysis of CD9 Expression in RASM Cells

Untreated and Ad-CD9 treated RASM cells were labeled with mouse IgG (MIgG) or CD9 specific mAb (mAb7). 
observed with two other cell lines, $\mathrm{CHO}$, and the mouse breast cancer cell line [Jyg $\mathrm{MC}(\mathrm{A})]$ transduced with the CD9 adenoviral construct (data not shown).

Adenoviral infection of cells or adenoviral mediated gene expression can have adverse effects on cell surface integrin expression. Additionally, expression of tetraspanins has also been reported to downregulate integrin expression. For example, $\mathrm{He}$ et al reported that in DU145 prostate cancer cell line, CD82 expression downregulates integrin $\alpha 6$ function by accelerating its internalization (15). CD82 and a few other tetraspanins (CD151, CD63, and CD37) possess a tyrosine-based endosomal/lysosomaltargeting motif in their C-terminal cytoplasmic region is thought to be responsible for internalization of integrins (15).

Therefore, our next objective was to investigate whether adenoviral mediated CD9 expression influenced cell surface integrin expression in RASM cells. To address this issue, we performed FCM experiments to compare the expression pattern of various integrins in adenoviral transduced RASM cells. Table 4.2 shows the mean fluorescence intensity (MFI) values obtained for integrin levels in untreated RASM cells or RASM cells infected with Ad-CD9 virus. As shown in Table 4.2 no significant differences in the MFI were detected for constitutive integrins $\alpha 1, \alpha 5$, and $\beta 1$. Cultured RASM cells did not express significant levels of integrin $\beta 3$ (Table 4.2, Figure 4.11).

Similar results were obtained with Ad-LacZ transduced RASM cells. The top panel of Figure 4.11 also depicts the histograms for the expression of $\alpha 5$ and $\beta 1$ integrin subunits in RASM cells before and after Ad-CD9 transduction. These results show that expression vector Ad-CD9 is capable of expressing a recombinant CD9 in RASM cells, and that viral transduction or CD9 expression did not alter key cell surface integrin 
Table 4.2 Comparison of CD9 and Integrin Expression in RASM Cells

\begin{tabular}{ccc}
\hline Antibody & Untransduced & $\begin{array}{c}\text { Ad-CD9 Transduced } \\
(\mathbf{1 0 0} \text { MOI) }\end{array}$ \\
\hline $\mathrm{mIgG}^{*}$ & $8.7 \pm 5.9$ & $6.9 \pm 2.1$ \\
$\mathrm{hIgG}^{* *}$ & $6.9 \pm 2.0$ & $6.6 \pm 0.2$ \\
Human $\mathrm{CD} 9(\mathrm{mAb} 7)$ & $6.3 \pm 0.6$ & $822 \pm 309$ \\
$\alpha 1(3 \mathrm{~A} 3)$ & $235.6 \pm 32.4$ & $234.2 \pm 24.1$ \\
$\alpha 5(\mathrm{HM} \alpha 5-1)$ & $18.5 \pm 4.7$ & $17.4 \pm 3.3$ \\
$\beta 1(\mathrm{HM} \beta 1-1)$ & $81.7 \pm 6.4$ & $78.7 \pm 7.6$ \\
$\beta 3(\mathrm{~F} 11)$ & $10.7 \pm 2.6$ & $10.3 \pm 2.4$ \\
\hline
\end{tabular}

Note: The numbers represent the mean fluorescence intensities \pm standard deviation from 3 independent experiments. * Mouse IgG ** Hamster IgG. 


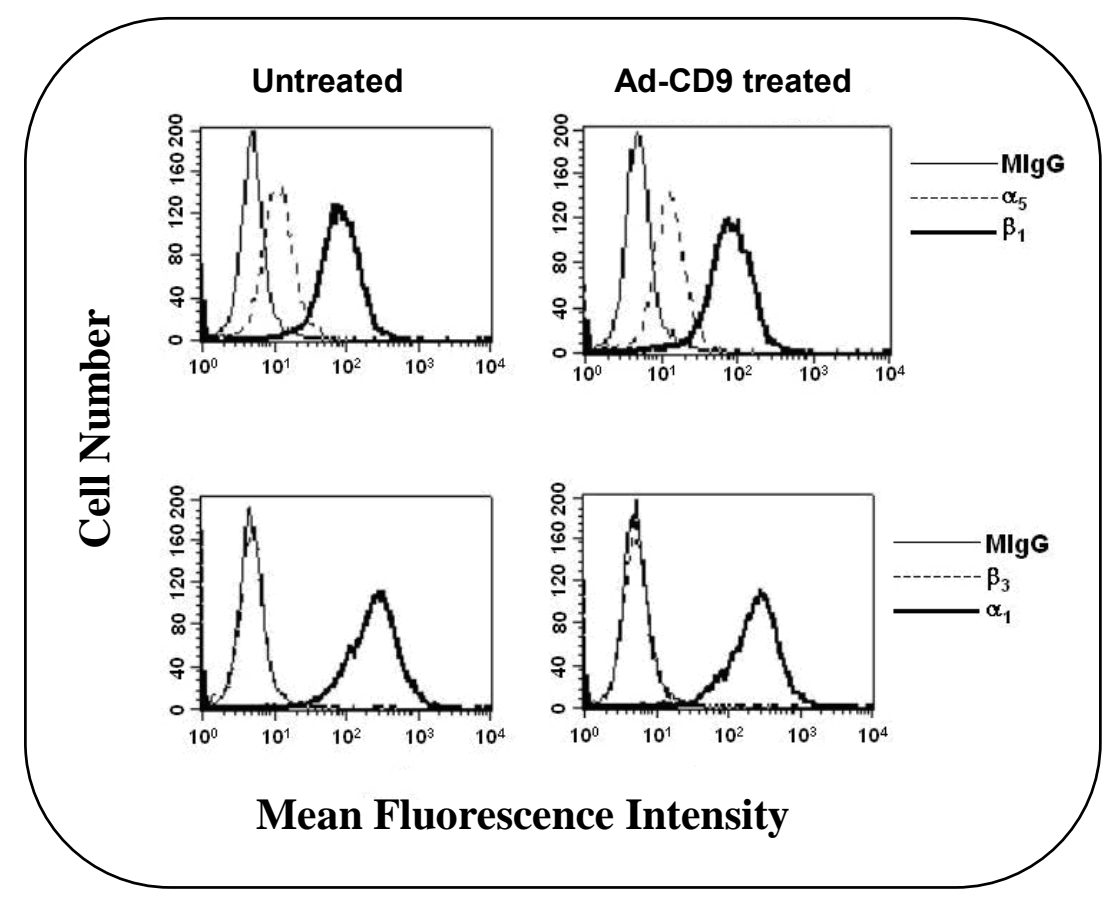

Figure 4.11 FCM Analysis of Integrin Expression in RASM Cells

Untreated and Ad-CD9 treated RASM cells were analyzed for the surface expression of Integrins. The histograms depict the expression of Integrins $\alpha 5, \beta 1$ (top panel), $\alpha 1$ and $\beta 3$ (bottom panel). MIgG: mouse IgG. 
expression. CD9 lacks the unique c-terminal tyrosine based internalization motif present in other tetraspanins (CD82, CD63, and CD151) and thus does not influence steady state levels of cell surface integrins.

\subsection{Effects of Transient Expression of CD9 on Akt Phosphorylation}

Since our data from CD9 stable transfection in $\mathrm{CHO}$ cells shows that $\mathrm{PI}-3 \mathrm{~K}$ is activated upon CD9 expression, we intended to study the affects of transient expression of CD9 on Akt phosphorylation. For these experiments, we utilized RASM cells treated with Ad-LacZ, Ad-CD9 viruses and untreated RASM cells. As seen in CHO cells, adhesion of RASM cells to FN caused significant increases in Akt phosphorylation on S473 residue as compared to the cells held in suspension (Figure 4.12).

CD9 expression in RASM cells significantly increased Akt phosphorylation in FN dependent manner. Total levels of Akt did not significantly differ between these cell types indicating that adenoviral transduction did not affect total Akt levels. The enhanced phosphorylation of Akt on S473 site in CD9 expressing cells was abolished when cells were pretreated with PI-3K inhibitor, LY294002 (Figure 4.12). Quantification by densitometry showed that phosphorylated Akt was approximately two fold higher in CD9 expressing cells as compared with the control cells (igure 4.13). These results suggest that PI-3K activation in $\mathrm{CD} 9$ expressing $\mathrm{CHO}$ cells was not due to an aberrant stable clone and that the effect is a specific CD9 linked phenomenon. 


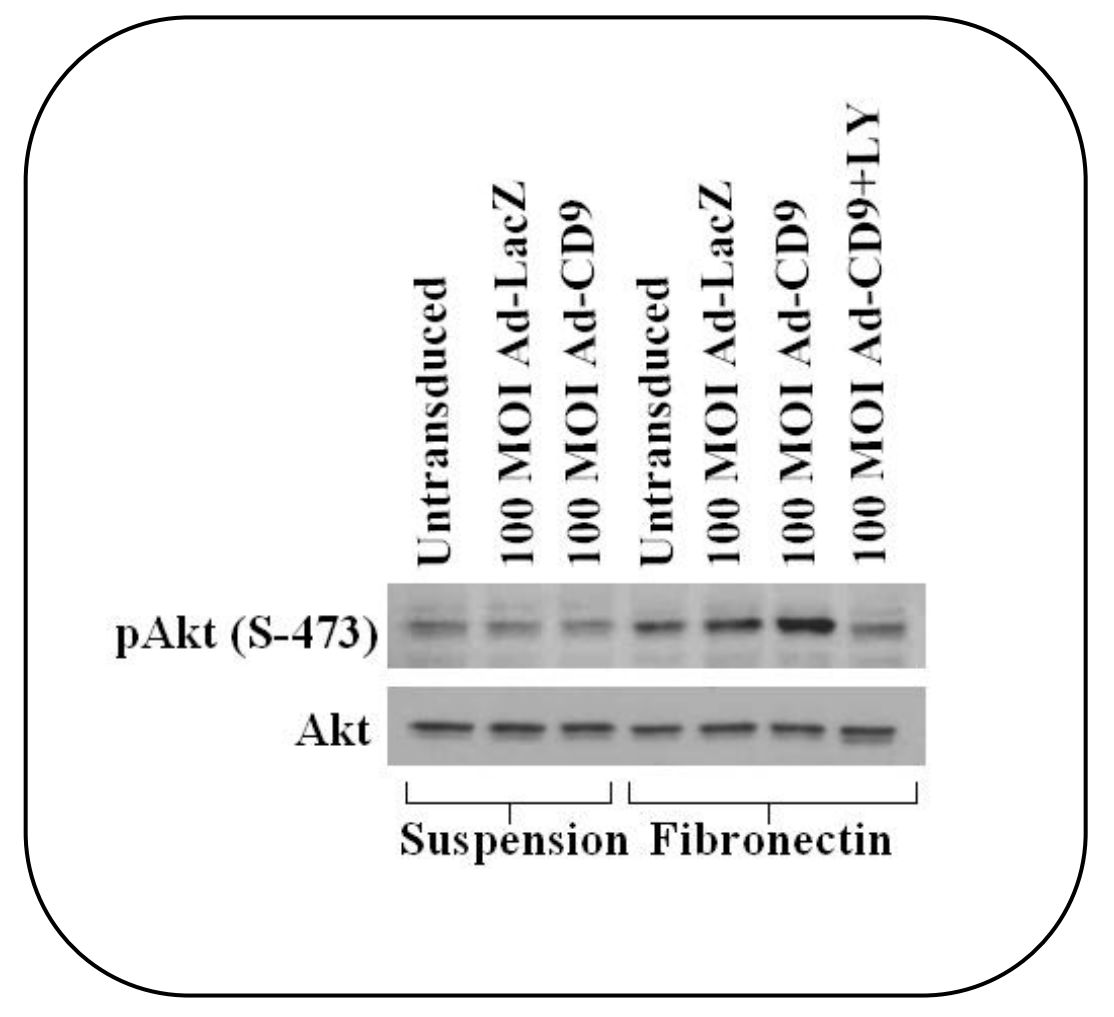

Figure 4.12 Effects of Transient Expression of CD9 on Akt Phosphorylation

Untransduced, Ad-CD9, Ad-LacZ transduced (MOI-100) RASM cells were either held in suspension or plated onto FN coated culture dishes for $3 \mathrm{hrs}$. Whole cell lysates were analyzed for phosphorylation of Akt on Serine 473 (top panel) and the same blot was stripped and reprobed for total Akt (bottom panel). The last lane represents lysates from Ad-CD9 transduced cells showing inhibition of Akt phosphorylation on Serine 473 with the PI-3K inhibitor LY294002. 


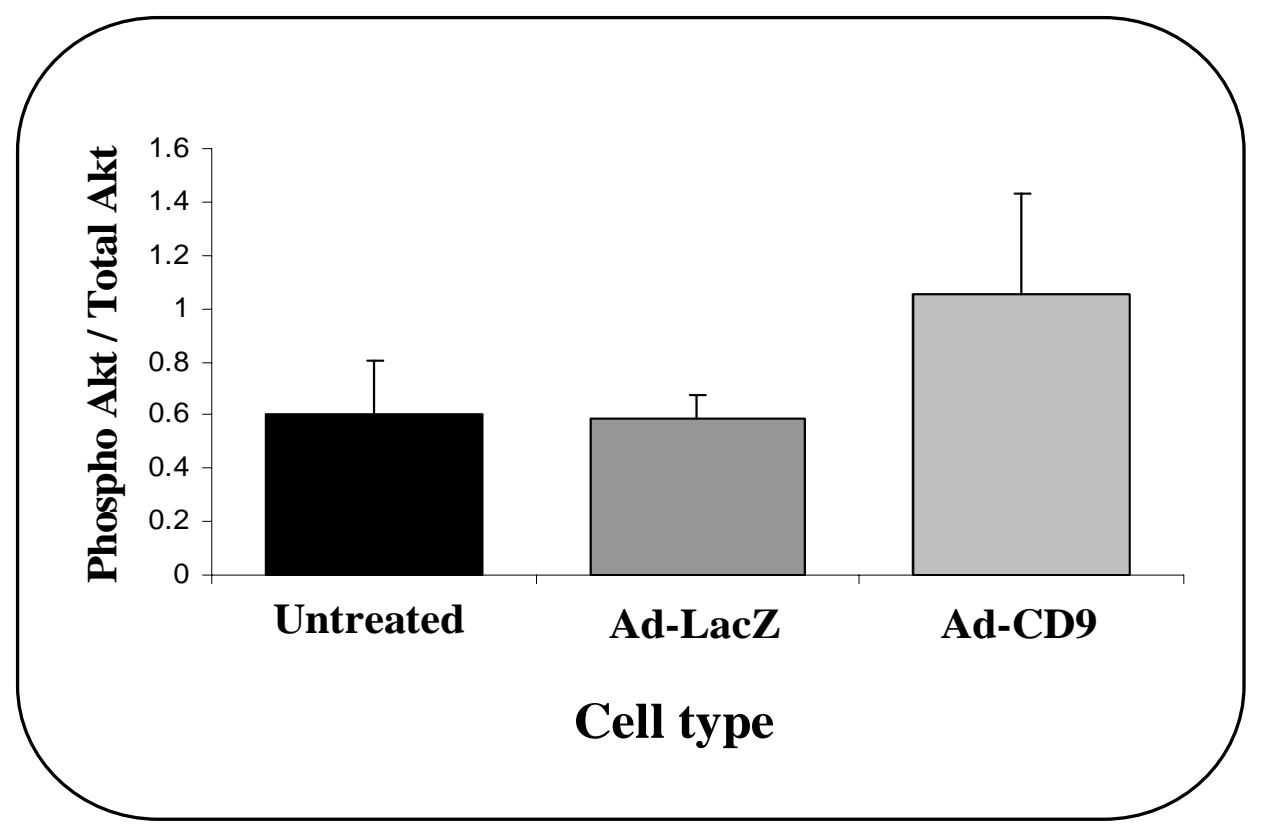

Figure 4.13 Quantification of Akt Phosphorylation in RASM Cells

Ratios of phosphorylated Akt and total Akt compared between untreated, Ad-LacZ treated and Ad-CD9 treated vascular smooth muscle cells plated onto FN for $3 \mathrm{hrs}(\mathrm{n}=3)$. The error bars represent the standard deviation. 


\subsection{The Role of p130Cas in CD9 Mediated Cell Motility}

It is well documented that cell adhesion to extracellular components leads to enhanced tyrosine phosphorylation of key signaling molecules. Tyrosine phosphorylation of high molecular weight proteins (above $100 \mathrm{kDa}$ ) has also been shown to activate PI$3 \mathrm{~K}$ pathway. In order to determine the potential effects of CD9 expression on protein tyrosine phosphorylation, we compared the adhesion associated tyrosine phosphorylation in Mock and A6 cells.

Lysates prepared from cells held in suspension or that were plated onto $\mathrm{FN}$ for 2 $\mathrm{hr}$ and $3 \mathrm{hr}$ were analyzed by Western blot with anti-phosphotyrosine mAb. Adhesion of both Mock and A6 cells to FN led to significant increase in tyrosine phosphorylation of a protein in the molecular weight range of 105-250. Additionally, A6 cells plated onto FN have siginificantly higher rates of tyrosine phosphorylation of this band as compared to the Mock cells. Since, both p130Cas (130 kDa) and FAK (120 kDa) are candidate molecules within this molecular weight range (263), we subsequently focused on studying these molecules (Figure 4.14).

p130Cas is an adapter protein that has been reported to be activated upon cell adhesion to $\operatorname{ECM}(263,264)$. Tetraspanins have been reported to mediate signaling via the p130Cas pathway. For example, expression of CD82, a metastasis suppressor tetraspanin, in prostate cancer cells led to down regulation of p130Cas total protein levels with concomitant inhibition of cell motility (39). Because of these observations, we investigated the possible involvement of p130Cas in CD9 mediated cell migration.

Firstly, to determine whether CD9 expression affected p130Cas expression, Western blot was performed on whole cell lysates prepared from Mock and A6 cells. 


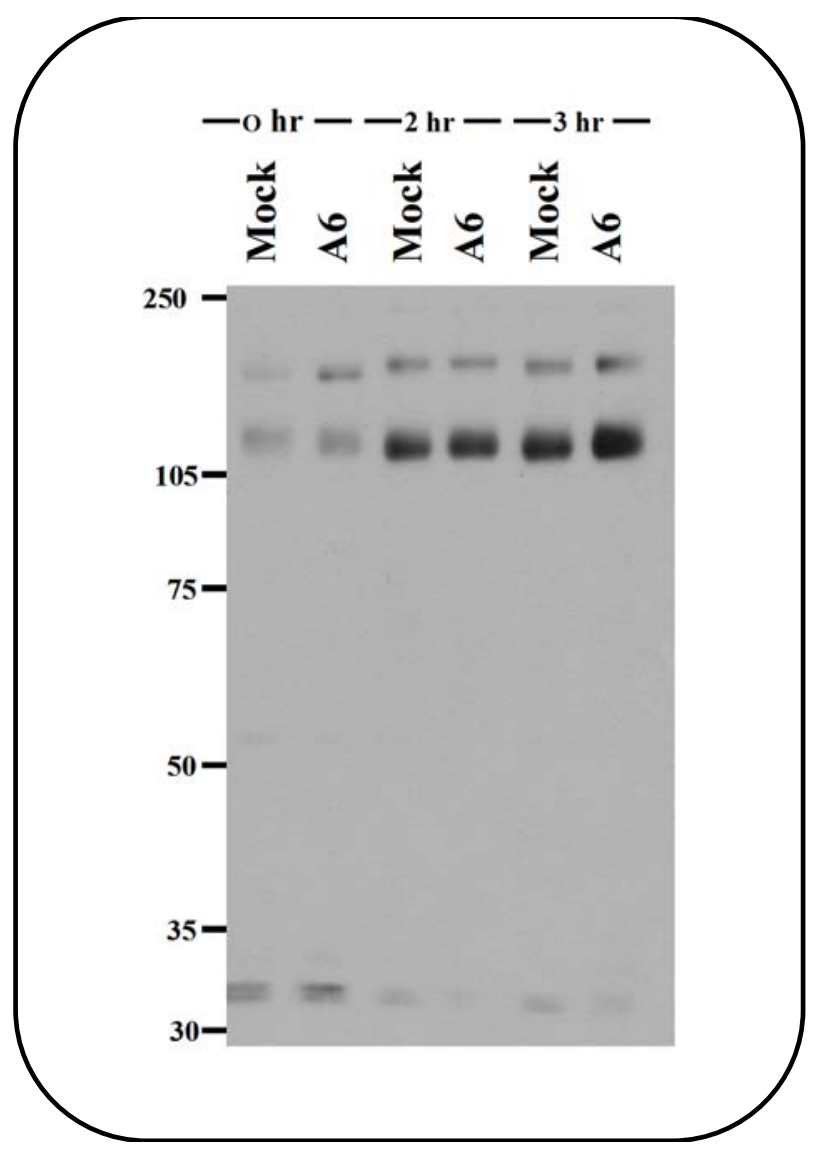

Figure 4.14 Analysis of Protein Tyrosine Phosphorylation in Mock and A6 Cells

Western blot analysis of cell lysates with anti-phosphotyrosine mAb showed that A6 cells have enhanced tyrosine phosphorylation of proteins in the molecular weight range 105$250 \mathrm{kDa}$. 
Figure 4.15 shows that levels of p130Cas were significantly higher in CD9 expressing cells as compared to the Mock cells. The total levels of Focal Adhesion Kinase (FAK) and actin did not differ between these two cell types (igure 4.15). Densitometry quantification of p130Cas and actin indicated that p130Cas levels in A6 cells were approximately 58\% higher in CD9 expressing cells as compared to the Mock cells (Figure 4.16).

FAK was shown to be tyrosine phosphorylated upon integrin activation (194, 265). Specifically, phosphorylation of FAK on tyrosine (Y) 397 has been shown to be responsible for its activation $(194,266)$ Therefore, we compared the adhesion dependent phosphorylation of FAK on Y397 residue in Mock and A6 cells. Although cell adhesion to FN enhanced phosphorylation of FAK on Y397, no significant differences were observed between Mock and A6 cells (data not shown).

Tyrosine phosphorylation of p130Cas creates docking sites for the $\mathrm{SH}$ domain containing proteins, that leads to activation of downstream signaling pathways. To understand whether the increase in p130Cas protein levels contributed to changes in its tyrosine phosphorylation state, the following experiments were performed. Mock and A6 cells were either held in suspension or were allowed to adhere to $\mathrm{FN}$ for the indicated time and cell lysates were prepared as described in the material and methods.

Subsequently, p130Cas was immunoprecipitated from cell lysates using specific $\mathrm{mAb}$ and the resulting immunoprecipitates were separated on SDS-PAGE. Tyrosine phosphorylation state of immunoprecipitated p130Cas was detected using antiphosphotyrosine antibody (RC-20HRP). No significant differences in tyrosine phosphorylation of p130Cas were observed between Mock and A6 cells held in 


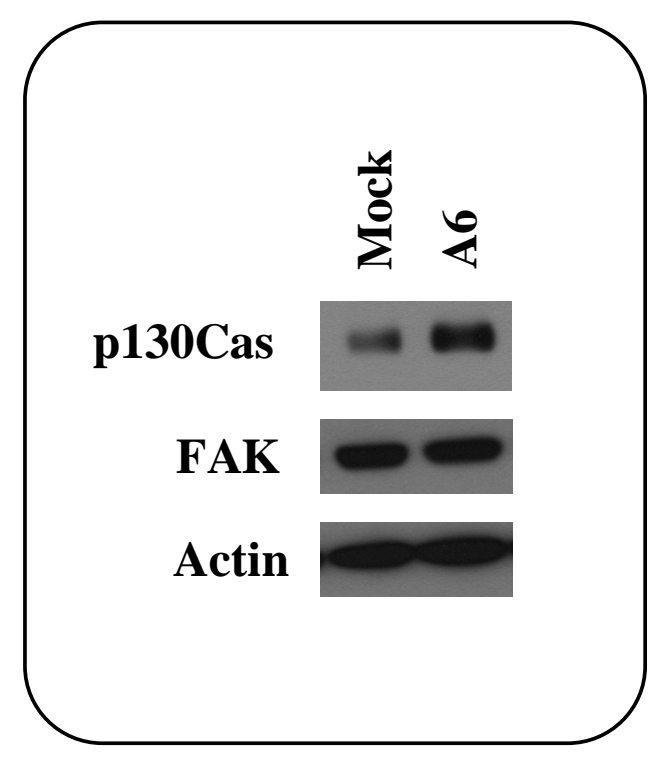

Figure 4.15 Western Blot Analysis of p130Cas Expression in Mock and A6 Cells

Whole cell lysates from Mock and A6 cells were analyzed for the expression of p130 Cas (top panel) FAK (middle panel) and total cellular actin (bottom panel). 


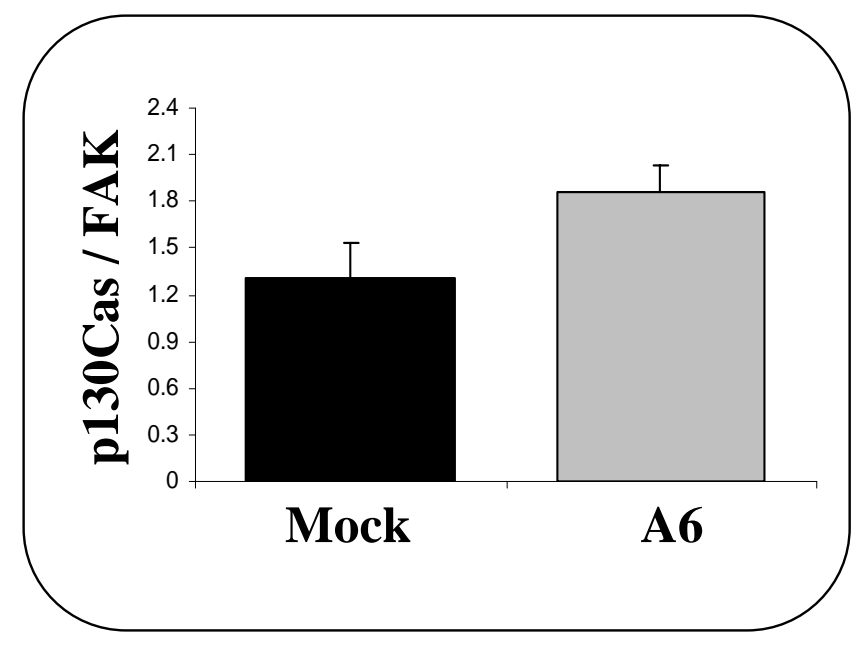

Figure 4.16 Quantitative Comparison p130Cas Expression in Mock and A6 Cells

Results of the scanning densitometry showing ratio between total p130Cas and total FAK. The error bars represent the standard deviation. 
suspension. However, in both Mock and A6 cells, p130Cas was increasingly tyrosine phosphorylated at $1 \mathrm{hr}$ and $2 \mathrm{hr}$ after cell adhesion to $\mathrm{FN}$ (Figure 4.17). In cells plated onto FN, corresponding to the increased levels of p130Cas in A6 cells, the extent of tyrosine phosphorylation of p130Cas was also significantly higher than in the Mock cells. These results clearly suggested that CD9 expression can up regulate p130Cas protein levels and tyrosine phosphorylation state.

p130Cas has been reported to play an important role in directional migration of cells to FN (267). Interestingly, CD82 mediated suppression of metastasis has been shown to be due to downregulation of p130Cas levels in DU-145 cells (39). As CD9 expression promotes directional migration of $\mathrm{CHO}$ cells to $\mathrm{FN}$ and also alters the total levels and phosphorylation of p130Cas, we next examined if p130Cas contributed to CD9 mediated haptotactic cell motility. To address the role of p130Cas in CD9 mediated motility, we utilized siRNA gene silencing methods to suppress the endogenous levels of p130Cas.

Since A6 cells have an up regulated p130Cas protein levels as compared to the Mock cell, the goal of p130Cas siRNA transfection experiments was to specifically suppress endogenous p130Cas levels in A6 cells to that seen in Mock cells and then to study its affects on cell migration. As a hamster specific sequence for the p130Cas was not available for designing siRNA duplexes, we utilized the mouse siRNA duplexes that were first tested for their potential to inhibit p130Cas message in the $\mathrm{CHO}$ cells.

Transient transfection experiments with control or the p130Cas siRNA duplexes were performed as described in Materials and Methods section. Transient transfection of Mock and A6 cells with p130Cas siRNA duplexes resulted in significant down regulation 


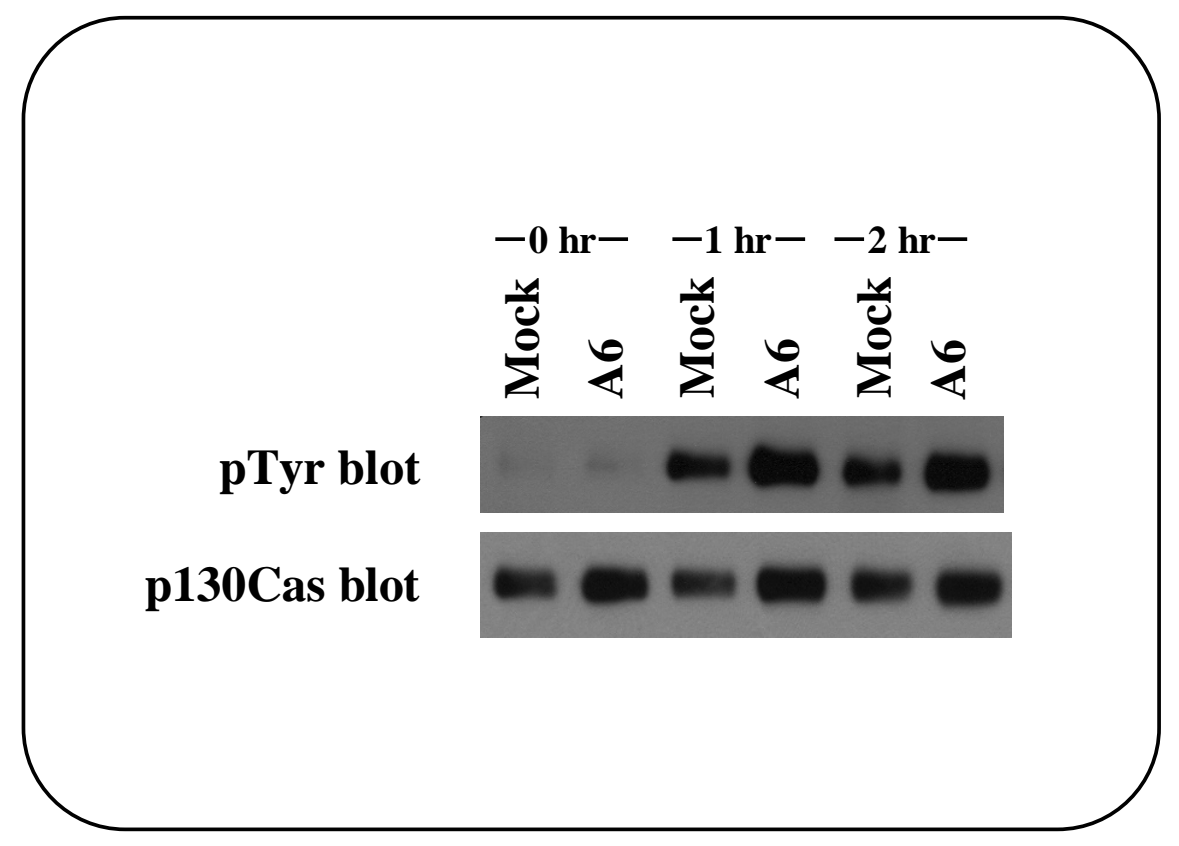

Figure 4.17 Western Blot Analysis of p130Cas Tyrosine Phosphorylation

p130Cas was immunoprecipitated from Mock, A6 cell lysates and Western blot was performed for tyrosine phosphorylation (pTyr) (top panel). The blots were subsequently stripped and re probed for total p130Cas (Bottom panel). 
of endogenous p130Cas protein levels. As expected, transfection of cells with the control siRNA did not alter the endogenous levels of p130Cas (igure 4.18). In order to demonstrate the specificity of siRNA duplexes, total cellular levels of actin were also examined. As shown in bottom panel of Figure 4.18, neither p130Cas nor the control siRNA transfections altered the total cellular levels of actin. These results demonstrated that hamster p130Cas has significant sequence homology to the mouse p130Cas and that mouse p130Cas siRNA duplexes can be successfully used to knock down p130Cas in CHO cells.

After validating the utility of mouse p130Cas siRNA duplexes in $\mathrm{CHO}$ cell model system, we further proceeded to investigate the effects of knockdown of p130Cas in CD9 mediated cell motility. To investigate this we performed haptotactic motility assays on FN. Twenty-four hrs after transfection with siRNA duplexes, Mock and A6 cells were harvested and subjected to transwell migration assays as described earlier. As expected, control siRNA transfection did not significantly affect the migration of Mock and A6 cell on FN and A6 cells still maintained a three fold higher migration than the Mock cells. No significant differences in cell migration were noted between A6 cells transfected with control or p130Cas siRNA (Figure 4.19).

Although overall p130Cas levels in lysates from A6 cells after p130Cas-siRNA transfection were reduced to those in the Mock cells (igure 4.18) transfected with control siRNA, A6 cells had 3-4 fold higher migration than Mock cells. Since the percentage of transfection efficiency using p130Cas siRNA duplex has not been estimated and because a majority of motile cells may not actually represent the p130Cas siRNA transfected cells, the above results should be interpreted with caution. Alternative 


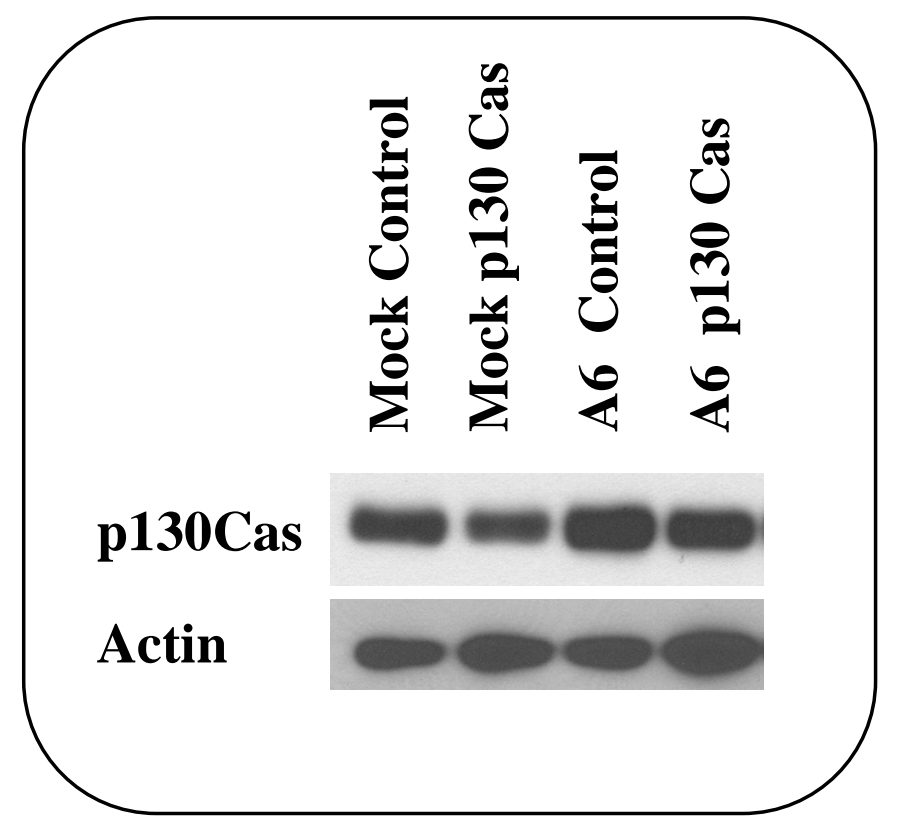

Figure 4.18 Western Blot Analysis after p130Cas siRNA Transfection

Levels of p130Cas (top panel) and actin (bottom panel) after transfection of Mock and A6 cells with either control siRNA duplex or the p130Cas SiRNA duplex. 


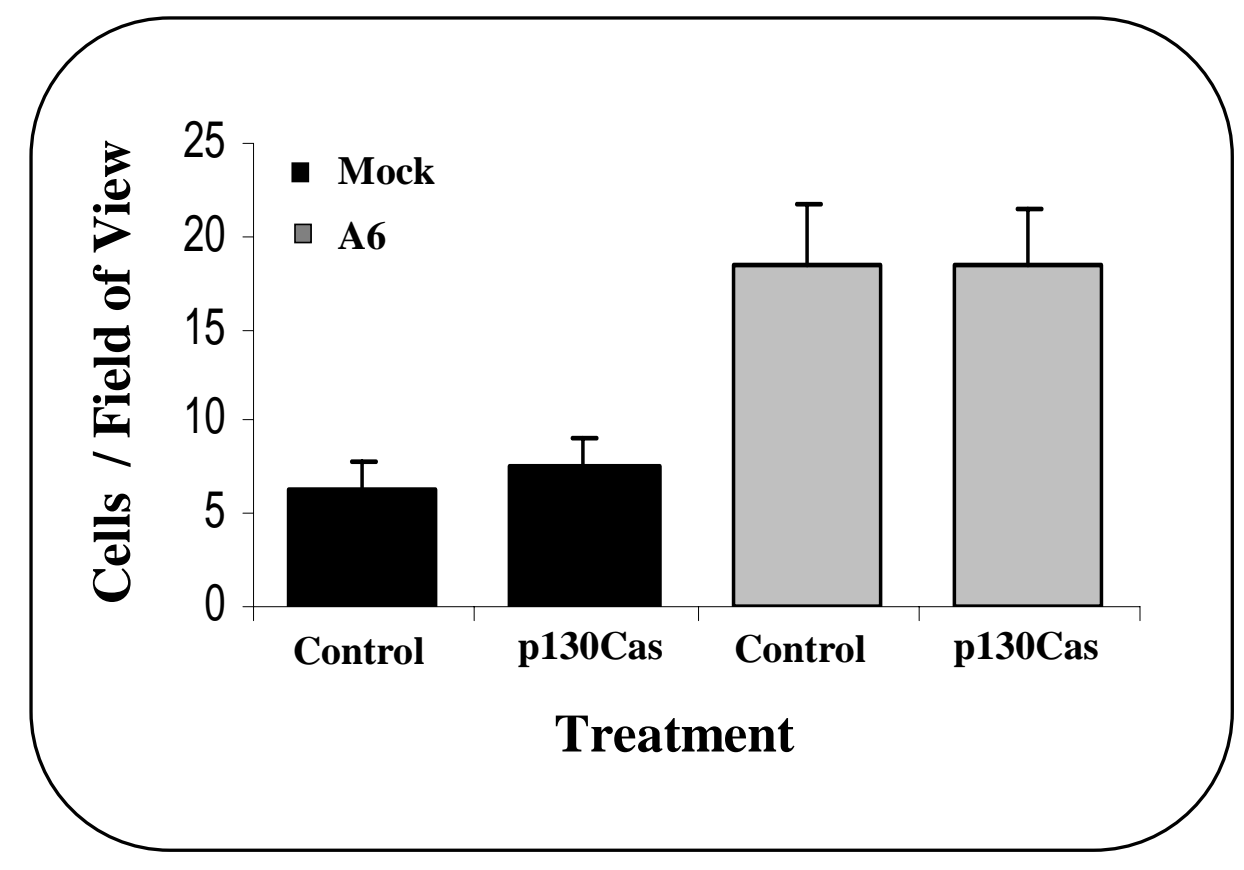

Figure 4.19 Motility of Mock and A6 cells after p130Cas siRNA Transfection

Mean cell migration of Mock and A6 cells after transient transfection with control siRNA duplex (Ctr) or the p130Cas siRNA duplex (p130Cas) $(n=3)$. The error bars represent the standard deviation. 
methods with high efficiency transfection rates such as the lentivirus vector mediated downregulation of p130Cas may conclusively prove the role of p130Cas in CD9 mediated phenotypes. Thus the functional implication of upregulated p130Cas in CD9 expressing cells remains to be elucidated.

\subsection{Physical Association of CD9 with Integrins}

Members of the TM4SF family proteins can associate with each other and with integrins to form multimeric complexes on the cell membrane often referred to as the "tetraspanin web" (9). The characteristic feature of the tetraspanin-integrin interactions is their sensitivity to disruption by stronger detergent solutions such as Triton-X 100 . Therefore, most tetraspanin-integrin associations are evident only in cell lysates prepared with mild detergent solutions such as Brij-98, Brij-99 and CHAPS. Few tetraspanins such as CD151 and CD81 retain the capability to interact with integrins even under stringent detergent conditions $(3,268)$. Thus within the tetraspanin web, CD151 and CD81 are thought to maintain primary or direct interactions with the integrins which are characterized by their specificity and stoichiometry.

It has been further hypothesized that other TM4SF members such as CD9 participate in primary complexes via secondary or indirect interactions (3). Secondary interactions, in contrast to primary interactions are sensitive to disruption by stronger detergents like Triton-X100. Although CD9 has been shown to associate with integrins containing $\beta 1$ and $\beta 3$ subunits, so far, there is no evidence showing CD9 is able to maintain direct interactions with these integrins. While CD9- $\beta 1$ associations have been demonstrated in virtually all the cell types tested (138), CD9- $\beta 3$ association seem to be 
restricted to platelets and hematopoietic cell lines (108). In a given tissue, the overall composition of tetraspanin web is determined by the expression pattern of both integrins and tetraspanins.

In the current study our initial goal was to optimize the conditions for IP using the mild detergent Brij-98. Using Brij-98 lysis buffer to solubilize the membrane proteins we studied the interaction of CD9 with integrins. Human platelets express both CD9 and $\beta 3$ integrin (GPIIb/IIIa) and hence were used to study CD9- $\beta 3$ interactions. Isolation, protein preparation and IP from platelet lysates were carried out as described in the material and methods. Figure 4.20 depicts the results of Western blot analysis for the detection of platelet integrins GPIIb and IIIa ( $\beta 3$ ) in mAb7 immunoprecipitates. Bands corresponding to both integrin subunits were detected in $\mathrm{mAb} 7$ immunoprecipitates. The mouse $\operatorname{IgG}$ negative control (MOPC-21) antibody did not immunoprecipitate either of these proteins.

IP of GPIIb from platelets with the 10E5 mAb resulted in co-IP of CD9. Similarly, IP of IIIa ( $\beta 3$ ) with AP3 mAb also resulted in co-IP of CD9. These results were consistent with several earlier observations demonstrating CD9-GPIIb/IIIa association and thus validated the use of Brij-98 lysis buffer to identify CD9-integrin interactions $(108,110)$. Since GPIIb/IIIa is the major integrin on platelets that express only moderate levels $\beta 1$ containing integrins, it would be interesting to identify the components of tetraspanin web in human platelets. In addition to CD9, platelets also express tetraspanin CD151. We anticipate that our experimental methods are valuable tools in understanding the complex nature tetraspanin-integrin interactions in human platelets. 


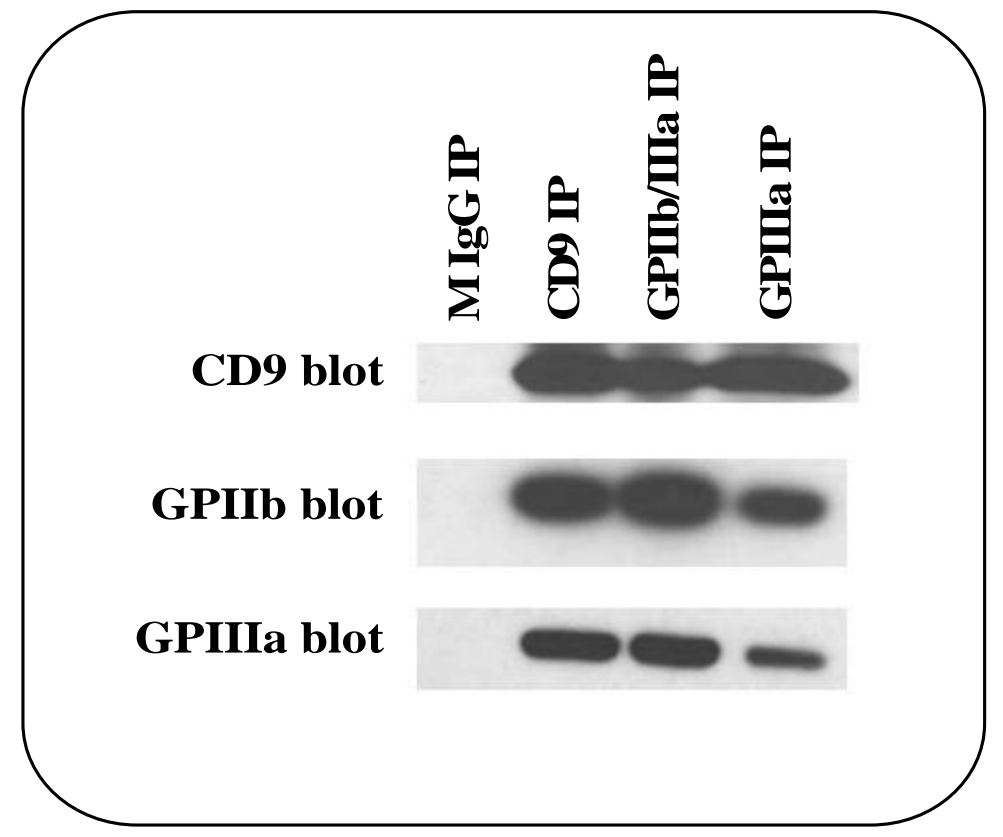

Figure 4.20 Association of CD9-GPIIb/IIIa in Platelets

Brij-98 lysates from the platelets were immunoprecipitated (IP) with antibodies specific for CD9, GPIIb/IIIa complex, and GPIIIa or the mouse IgG control (MIgG). Immunecomplexes were subsequently analyzed by Western blot for CD9 (top panel), GPIIb (middle panel) and GPIIIa (bottom panel). 
The association between CD9 and $\beta 1$ was studied using cultured cells. In HT1080 cells, only small amounts of endogenous CD9 were expressed as detected by FCM. Therefore, we generated stable CD9 expressing cell line (CD9-HT1080) as described in materials and methods. Lysates from Mock transfected HT1080 cells and CD9-HT1080 cells were prepared and IP experiments were performed as described in materials and methods. Figure 4.21 shows the results of these experiments where presence of $\beta 1$ integrin was detected by Western blot analysis of immunoprecipitated proteins.

As expected the mouse $\mathrm{IgG}$ control $\mathrm{mAb}$ did not immunoprecipitate integrin $\beta 1$ from cell lysates. A band corresponding to $\beta 1$ integrin was detected from both Mock and CD9 HT1080 lysates, where anti-human $\beta 1 \mathrm{mAb}$, TS2/16, was used for IP. IP of CD9HT1080 cell lysates with mAb7 resulted in $\beta 1$ co-IP. As Mock HT-1080 cells expressed only insignificant levels of $\mathrm{CD} 9$, a band corresponding to $\beta 1$ integrin was not detected in mAb7 immunoprecipitates from these cells. These results further validated the usability of Brij-98 detergent to demonstrate CD9-integrin interactions.

After demonstrating CD9- $\beta 1$ interaction in HT1080 cells, we furthered our study to demonstrate CD9 interactions with integrin $\alpha 5 \beta 1$. So far CD9- $\alpha 5 \beta 1$ interactions have been demonstrated in very few cell types such as trophoblasts and myocytes $(99,135)$. To determine whether $\alpha 5 \beta 1$, associates with CD9, we carried out experiments using RASM cells transduced with the Ad-CD9 vector. The relationship between $\alpha 5 \beta 1$ and CD9 was examined by IP experiments where untransduced RASM cells served as negative controls.

As shown in Figure 4.22, CD9 was immunoprecipitated with anti-human CD9 $\mathrm{mAb}(\mathrm{mAb} 7)$ only from cells expressing recombinant human CD9 but not from the 


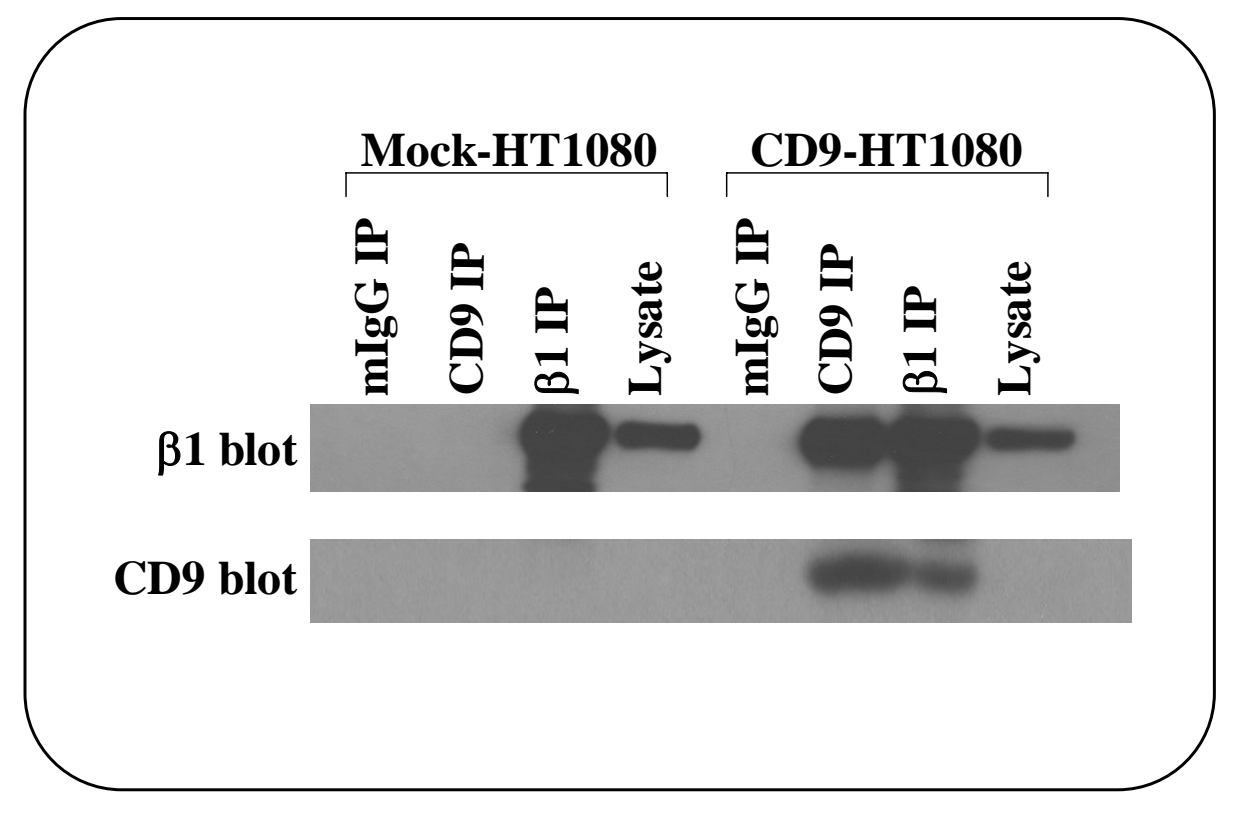

Figure 4.21 Association of CD9 and Integrin $\beta 1$ in HT1080 Cells

Brij-98 lysates from Mock-HT1080 and CD9-HT1080 cells were immunuprecipitated with mouse $\mathrm{IgG}(\mathrm{mIgG}), \mathrm{CD} 9$ and integrin $\beta 1 \mathrm{mAbs}$ as described in Materials and Methods. Immunecomplexes were analyzed for integrin $\beta 1$ (top panel) and CD9 (bottom panel). 


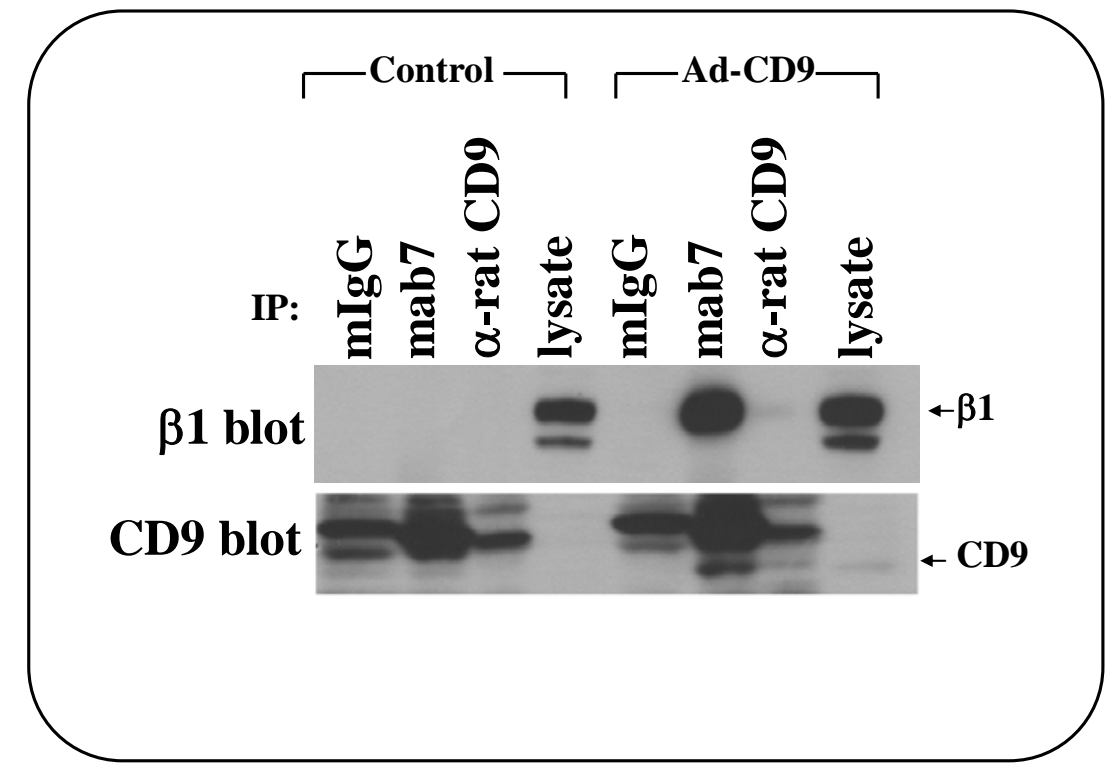

Figure 4.22 Association of CD9 with Integrin $\beta 1$ in RASM Cells

Brij-98 lysates from untransduced (control) and Ad-CD9 transduced (Ad-CD9) were immunoprecipitated with mouse IgG (mIgG) anti-human CD9 (mAb7), anti-rat CD9 $\mathrm{mAb}(\alpha-$-rat CD9) and immunoprecipitates were analyzed for integrin $\beta 1$ (top panel) and CD9 (bottom panel). 
control cells. MOPC-21 (mIgG control) did not immunoprecipitate CD9 either from control cells or from CD9 expressing RASM cells. No significant differences in $\alpha 5$ and $\beta 1$ integrin subunit protein levels were detected between the control cells and Ad-CD9 transduced cells. Western blot analyses of mAb7 immunoprecipitates indicated that both the integrin subunits $\alpha 5$ and $\beta 1$ co-immunoprecipitated with CD9 (Figure 4.22, Figure 4.23).

Two bands corresponding to mature and immature chains of integrin $\beta 1$ were detected by Western blot under reducing conditions (Figure 4.22). Sequential IP and Western blotting with two different mAbs (HM $\beta 1-1$, BD clone-18) to $\beta 1$ confirmed that these two bands indeed corresponded to the $\beta 1$ integrin subunit. The higher molecular weight band corresponded to the mature (fully glycosylated) form of integrin $\beta 1$ and the lower molecular weight band the immature (partially glycosylated) form $(269,270)$. Only the mature integrin $\beta 1$ was detected in CD9 immunoprecipitates. As expected, neither CD9, $\alpha 5$, nor $\beta 1$ subunits co-immunoprecipitated with CD9 from the control cells.

These results confirmed our hypothesis that CD9 is in association with integrin $\alpha 5 \beta 1$. Additionally, the observation that CD9 only immunoprecipitated higher molecular weight forms of $\beta 1$ suggested that maturation of $\beta 1$ by post translational modification such as glycosylation is an essential requirement for CD9- $\beta 1$ interactions. An $\alpha 5 \beta 1-C D 9$ association was not detected in higher stringency Triton X-100 derived cell lysates (data not shown). Susceptibility of CD9- $\alpha 5 \beta 1$ complexes for disruption by stronger detergents is consistent with earlier finding and whether CD9 requires other tetraspanins such as CD151 to maintain $\alpha 5 \beta 1$ interaction remains to be investigated. 


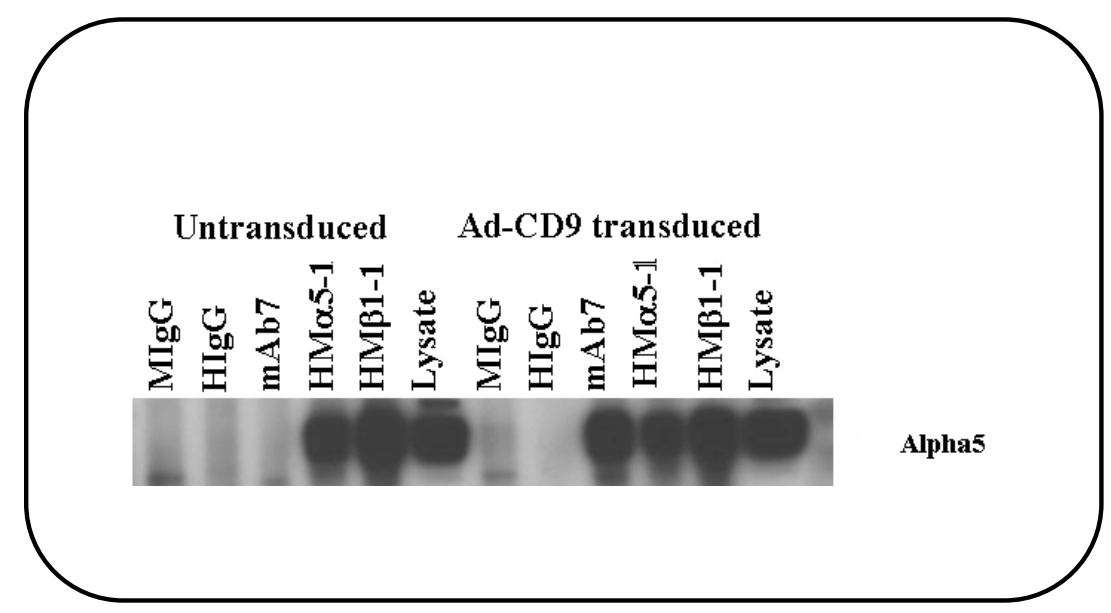

Figure 4.23 Association of CD9 with Integrin $\alpha 5$ in RASM Cells

Brij-98 lysates from untransduced (control) and Ad-CD9 transduced (Ad-CD9) were immunoprecipitated with mouse $\operatorname{IgG}(\mathrm{mIgG})$, hamster IgG (HIgG), anti-human CD9 (mAb7), anti-rat $\alpha 5 \mathrm{mAb}(\mathrm{HM} \alpha 5-1)$ and anti-rat $\beta 1 \mathrm{mAb}(\mathrm{HM} \beta 1-1)$ and immunoprecipitates were analyzed for integrin $\alpha 5$. 
Although ectopic expression of proteins in cultured cells is an invaluable tool in understanding the characteristics of a protein, exogenous expression systems are prone to false positive findings. For example, non-specific protein-protein interactions can occur when proteins are expressed above the physiological levels directly contributing to the false positive results. To circumvent these problems, we tested CD9- $\alpha 5 \beta 1$ interactions using a cell line where both CD9 and $\alpha 5 \beta 1$ are expressed endogenously. Testing of several cell lines available to us showed that mouse embryonic fibroblasts (MEF) expressed significant levels of both CD9 and $\alpha 5 \beta 1$ and therefore were used for subsequent testing.

Figure 4.24 shows the results of FCM experiments performed on MEF for the detection of CD9, $\alpha 5$, and $\beta 1$. As shown in the left panel of Figure 4.24, mAbs specific for integrin subunits $\alpha 5, \beta 1$ caused a right shift in the fluorescence intensity as compared to the hamster IgG isotype (control) antibody. The right panel of Figure 4-24 depicts similar right shift in fluorescence intensity observed with the rat monoclonal antibody against mouse CD9 (KMC.8). These results confirmed the expression of integrin $\alpha 5, \beta 1$ and CD9 in MEF.

In order to demonstrate the physical association between CD9 and $\alpha 5 \beta 1$ in MEF, $\alpha 5, \beta 1$ and CD9 were immunoprecipitated from cell lysates prepared with Brij-98 lysis buffer. The presence of $\alpha 5$ in the immunoprecipitates was detected using a polyclonal antibody against $\alpha 5$. As shown in Figure 4.25, a band corresponding to $\alpha 5$ was detected in both $\alpha 5, \beta 1 \mathrm{mAb}$ immunoprecipitates. As expected, the hamster IgG isotype matched antibody did not immunoprecipitate $\alpha 5$. Supporting our findings from RASM cell model 


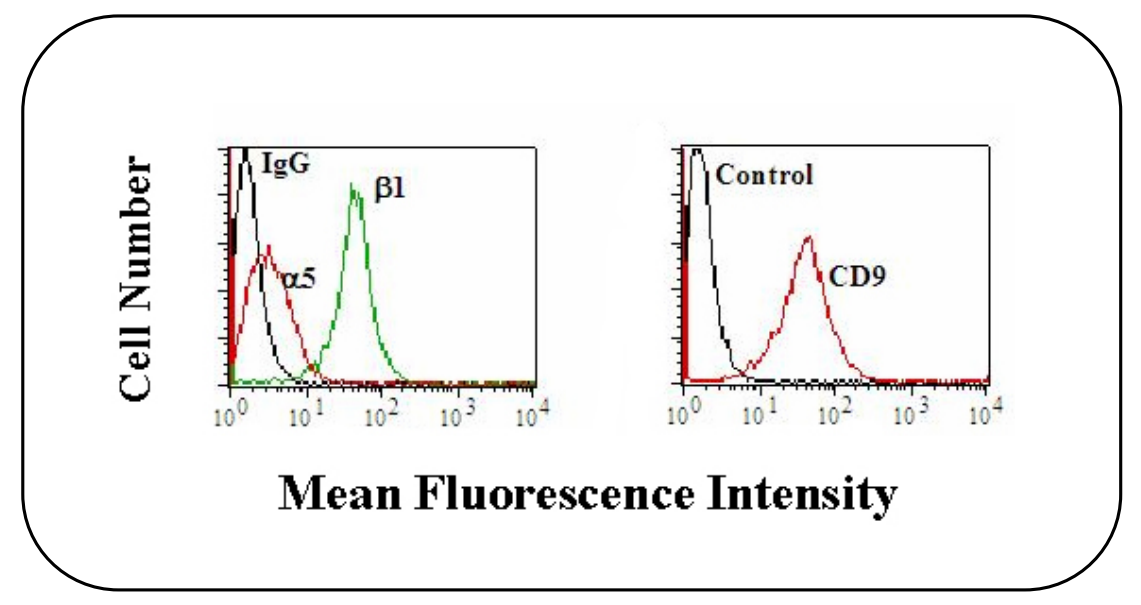

Figure 4.24 FCM Analysis of CD9 and $\alpha 5 \beta 1$ Expression in MEF

Flow cytometry (FCM) analysis of mouse embryonic fibroblast (MEF) cells for the expression of integrins $\alpha 5, \beta 1$ (left panel) and CD9 (right panel). 


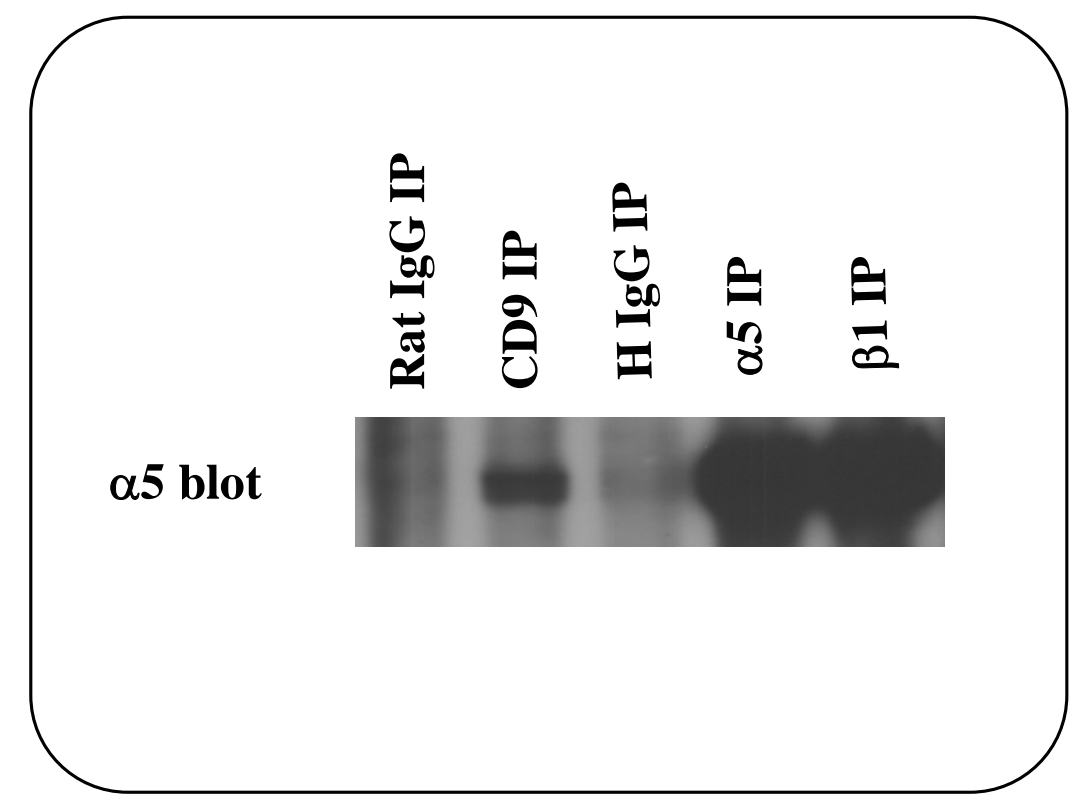

Figure 4.25 Association of CD9 with Integrin $\alpha 5$ in MEF

Brij-98 lysates from mouse embryonic fibroblasts (MEF) were immunoprecipitated with Rat IgG, anti-CD9, hamster IgG (HIgG), anti- $\alpha 5$ and anti- $\beta 1$ antibodies and immunoprecipitates were analyzed by Western blot for $\alpha 5$ integrin. 
systems, a band corresponding to $\alpha 5$ integrin was also detected in anti-CD9 mAb (KMC.8) immunoprecipitates (Figure 4.25).

Previous work from our laboratory using confocal laser scanning microscopy (CLSM) experiments showed that CD9 co-localizes with $\beta 1$ integrin in CHO cells (200). Co-localization experiments with CLSM, although prove the spatial proximity of proteins do not confirm the physical association between proteins. Also the regions of CD9 required for interaction with $\alpha 5 \beta 1$ appear to be highly conserved as recombinant human CD9 was able to associate with rat $\alpha 5 \beta 1$ integrin.

Our findings underscore an important regulatory role of CD9 in vascular smooth muscle cell (VSMC) functions. We show that in VSMC CD9 associates with integrin $\alpha 5 \beta 1$ and can activate $\alpha 5 \beta 1$ dependent signaling pathways. CD9 expression has been shown to correlate with the proliferative phenotype of VSMC (93). Proliferative phenotype of VSMC is predominantly seen in the injured vessel where there is also extensive $\alpha 5 \beta 1$ dependent ECM reorganization (271). Based on our observations, we hypothesize that in VSMC, increases in CD9 expression have potential functional implications regulating vascular injury response.

\subsection{The Role of Integrins in CD9 Mediated Cell Motility}

Although it is well known that CD9 and other tetraspanins associate with a variety of integrins, it is in unknown whether tetraspanins can directly regulate integrinfunctions. Tetraspanin CD151 has been recently shown to stabilize the active conformation of integrin $\alpha 3 \beta 1$ and upregulate its ligand binding activity (206). Several of CD9 induced phenotypic changes in cells are specific for FN that is known to be a classical receptor for 
$\alpha 5 \beta 1$. Our finding that CD9 is in complex with integrin $\alpha 5 \beta 1$ further prompted us investigate the contribution of this integrin in CD9 mediated phenotypic changes.

In addition to integrins, CD9 has also been shown to interact with growth factor related receptors. Expression of $\mathrm{CD} 9$ has been shown to up regulate the activity of membrane bound heparin binding epidermal growth factor-like growth factor (Pro-HBEGF) (33). To investigate whether CD9 promoted motility may be due to modulation of growth factor receptors responding to their soluble ligands, the following experiments were performed. One set of Mock and A6 cells were maintained in RPMI medium containing $1 \%$ FBS for $18 \mathrm{hrs}$ to eliminate the affects of residual growth factors in serum. Subsequently, the migration potential of these cells were compared to the Mock and A6 cells routinely cultured in RPMI containing 10\% FBS.

When the motility assay results were compared, the overall migratory potential of Mock and A6 cells cultured in RPMI and 1\% FBS media for 18 hrs was found to be $15 \%$ less than the motility of cells cultured in complete growth media. Despite elimination of residual growth factor effects, A6 cells showed more than a $250 \%$ increase in migratory rate as compared with the Mock cells (Figure 4.26). The ability of A6 cells to retain their pro-migratory phenotype under serum deprived conditions suggested that CD9 mediated motility in $\mathrm{CHO}$ cells is a phenomenon that is independent of effects of growth factors in the serum. This prompted us to investigate the role of integrins in CD9 mediated cell motility.

Integrin $\alpha 5 \beta 1$ is expressed endogenously in $\mathrm{CHO}$ cells and has been shown to regulate cell adhesion and migration on $\mathrm{FN}(147,201)$. Since CD9 also induces specific phenotypic changes on $\mathrm{FN}$ in $\mathrm{CHO}$ cells and because $\mathrm{CD} 9$ associates with $\alpha 5 \beta 1$, we 


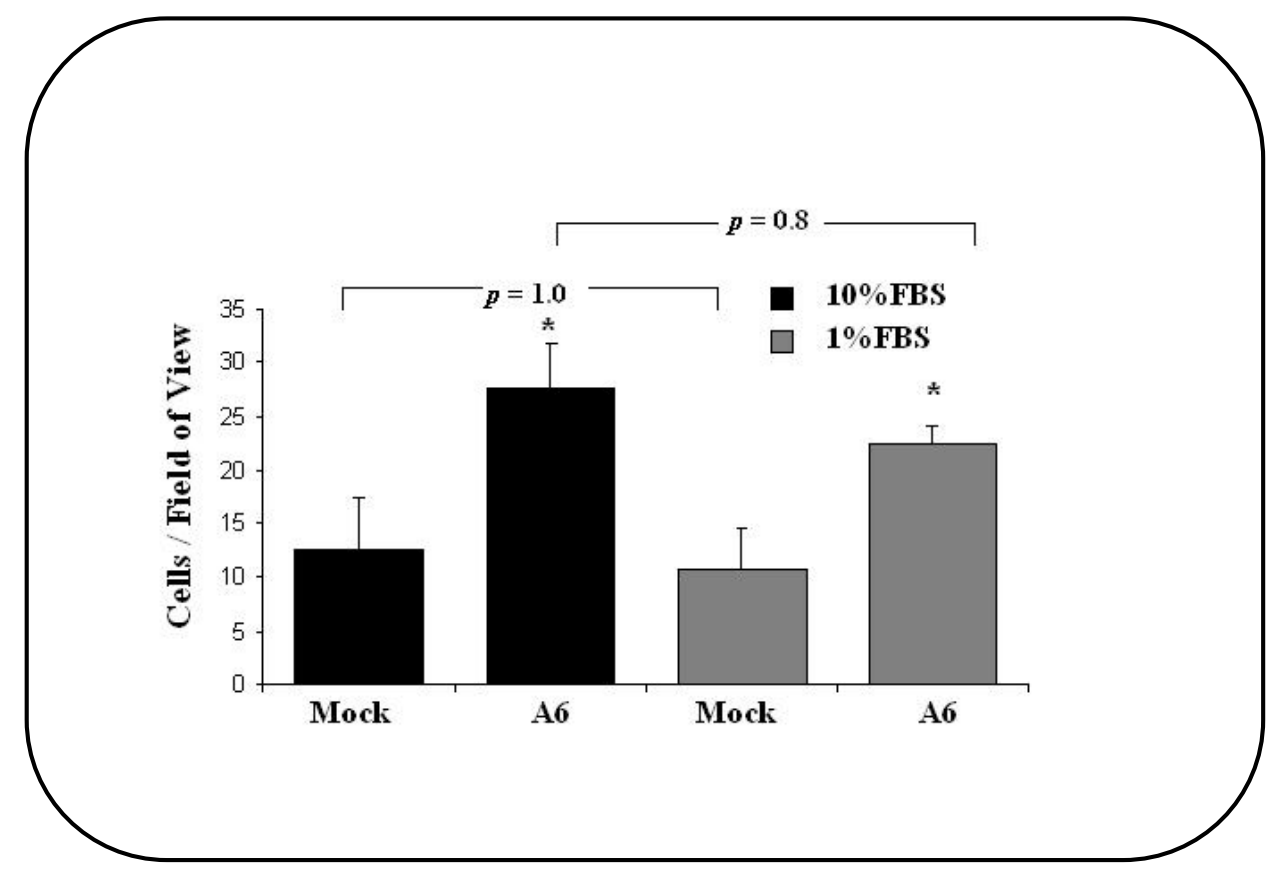

Figure 4.26 Effects of Serum on Mock and A6 Cell Migration

Migration of Mock and A6 cells grown in 10\% fetal bovine serum (FBS), 1\% FBS. Cells were allowed to migrate for $3 \mathrm{hrs}$ and cells were counted in 5 random high power fields $(\mathrm{n}=3) .{ }^{*}$ represents $\mathrm{p}<0.05$ compared to the respective Mock cells. The error bars represent the standard deviation. 
investigated the importance of integrin $\alpha 5 \beta 1$-FN binding in CD9 promoted haptotactic cell migration. Cook et al,1999 have previously shown that the $\alpha 5 \beta 1$ inhibitory antibody (PB1) reversed the characteristic spread phenotype induced by CD9 in CHO cells (173). We utilized a similar strategy to understand the importance of integrin $\alpha 5 \beta 1$ in CD9 promoted motility.

Monoclonal antibodies specific for integrin $\alpha 5 \beta 1$ (PB1) and subunit $\beta 1$ (7E2) were developed and subsequently characterized in CHO cells (147). PB1 has been shown to inhibit $\alpha 5 \beta 1$-mediated functions in $\mathrm{CHO}$ cells on FN (201). To test the contribution of $\alpha 5 \beta 1$ in CD9 promoted cell migration on FN, haptotactic motility experiments were performed using Mock and A6 cells pretreated with the $\alpha 5 \beta 1$ blocking mAb, PB1. As a negative control Mock and A6 cells treated with the isotype matched mAb (MOPC-21) were used.

As shown in Figure 4.27, treatment of cells with the MOPC-21 did not significantly inhibit A6 cell migration. The data shown are the results from the highest concentration of MOPC-21 used in the motility experiments. Even at concentrations as high as $100 \mu \mathrm{g} / \mathrm{ml}$, MOPC-21 did not inhibit CD9 promoted cell migration ( $\mathrm{p}=1.00$ as compared with the untreated control). However, treatment of A6 cells with $5 \mu \mathrm{g} / \mathrm{ml}$ of the PB1 antibody significantly inhibited ( $\mathrm{p}=0.007$ as compared with the $\mathrm{mIgG}$ control) CD9 promoted migration on FN. At $5 \mu \mathrm{g} / \mathrm{ml}$ concentration of PB1 Mock cell migration was only moderately effected.

Since integrin mediated functions are dependent on the activation state of integrin we next wanted to investigate whether CD9 directly modulates integrin $\beta 1$ conformation. In cultured cells, integrins can be activated by treating cells with divalent cations such as 


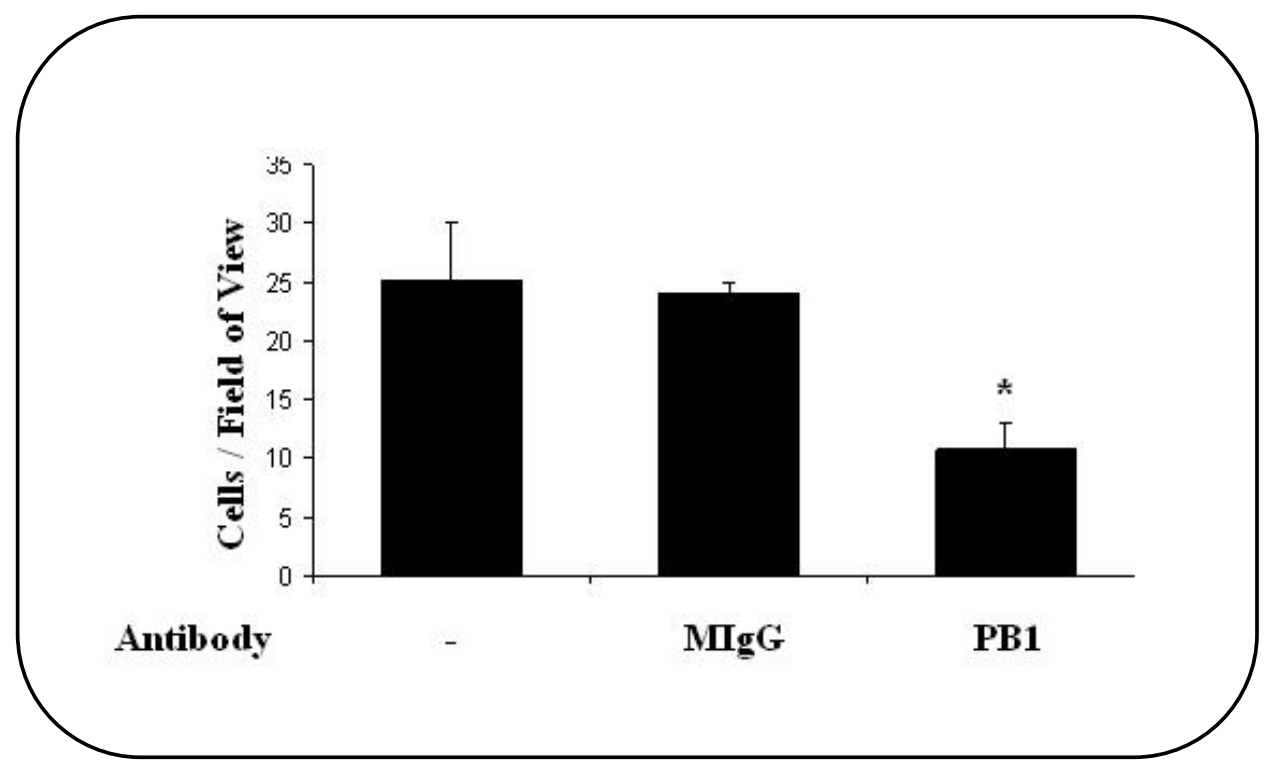

Figure 4.27 Effects of $\alpha 5 \beta 1$ Blocking Antibody (PB1) on A6 Cell Migration

Mean cell migration of untreated A6 cells (-) and A6 cells treated with mouse IgG control $(\mathrm{MIgG})$ or the $\alpha 5 \beta 1$ blocking antibody (PB1). * represents $\mathrm{p}<0.05$ as compared to the MIgG treated group $(n=3)$. The error bars represent the standard deviation. 
$\mathrm{Mn}^{2+}$ and $\mathrm{Ca}^{2+}$ or with ligand mimetic peptides (211-213). Integrins activated by several of these reagents expose newer epitopes known as the ligand induced binding sites (LIBS). LIBS can be specifically recognized by mAbs known as LIBS antibodies that have a characteristic high affinity binding to the active conformation of integrins.

Several $\beta 1$ specific LIBS antibodies have been generated to study its activation state. Most of these antibodies are generated against either mouse or human $\beta 1$ integrin thus limiting their usage in other animal model systems. Since we intended to investigate $\beta 1$ activation state in $\mathrm{CHO}$ cells, we initially screened various mouse specific LIBS antibodies to bind hamster $\beta 1$ integrin. Among the antibodies screened, only B44antibody specifically recognized hamster $\beta 1$ in its active conformation. B44 antibody was previously shown to recognize active conformation $\beta 1$ integrin $(213,272)$.

The RGDS sequence corresponds to a highly conserved region of the ECM ligand FN and has been shown to activate $\alpha 5 \beta 1$ complex. Therefore, experiments were performed where relative binding of B44 antibody to Mock and A6 cells was tested in the presence of ligand-mimetic RGDS peptide. This allowed us to directly compare the effects of CD9 expression on $\beta 1$ conformation state. Mock and A6 cells were first treated with either RGDS or RGES (negative peptide control) and then allowed to bind the B44 antibody. A positive signal from B44 binding was detected using a GAM-FITC secondary antibody.

Our results indicate that treatment of cells with the negative control RGES peptide did not induce a significant activation of $\beta 1$ integrin. Based on binding of B44 to RGES treated cells we observed that both Mock and A6 cells expressed similar levels of $\beta 1$ integrin on their surface. However, in the presence of integrin activating peptide RGDS, 
both Mock and A6 cells had significantly higher binding of B44 antibody as compared to the respective RGES treated control group.

In support of our hypothesis that CD9 can potentially influence the conformation of $\beta 1$, there was a significantly higher binding of B44 antibody in A6 cells (Figure 4.28) as compared to the Mock cells $(\mathrm{n}=5, \mathrm{p}<0.05)$. Although the above results clearly suggest that enhanced B44 binding is observed only in the presence of ligand-mimetic peptide RGDS, our findings do not address the mechanism by which CD9 can modulate conformation of $\beta 1$. It is yet to be determined whether CD9 by virtue of physical association with $\beta 1$ directly contributes to the stabilization of active conformer of $\beta 1$.

In summary, these data show that $\mathrm{CD} 9$ expressing cells retain their pro-migratory phenotype in the absence of a residual growth factor effect and suggest that CD9 promoted migration is due to its influence on adhesion molecules rather than growth factor receptors. Since, anti- $\alpha 5 \beta 1$ antibody PB1 inhibited CD9 promoted migration, it can be concluded that $\alpha 5 \beta 1$ interactions with its ligand FN are essential for CD9 promoted migration and that CD9 can serve to upregulate $\alpha 5 \beta 1$ mediated events. Our results emphasize an important regulatory role for $\mathrm{CD} 9$ in modulating integrin conformation. 


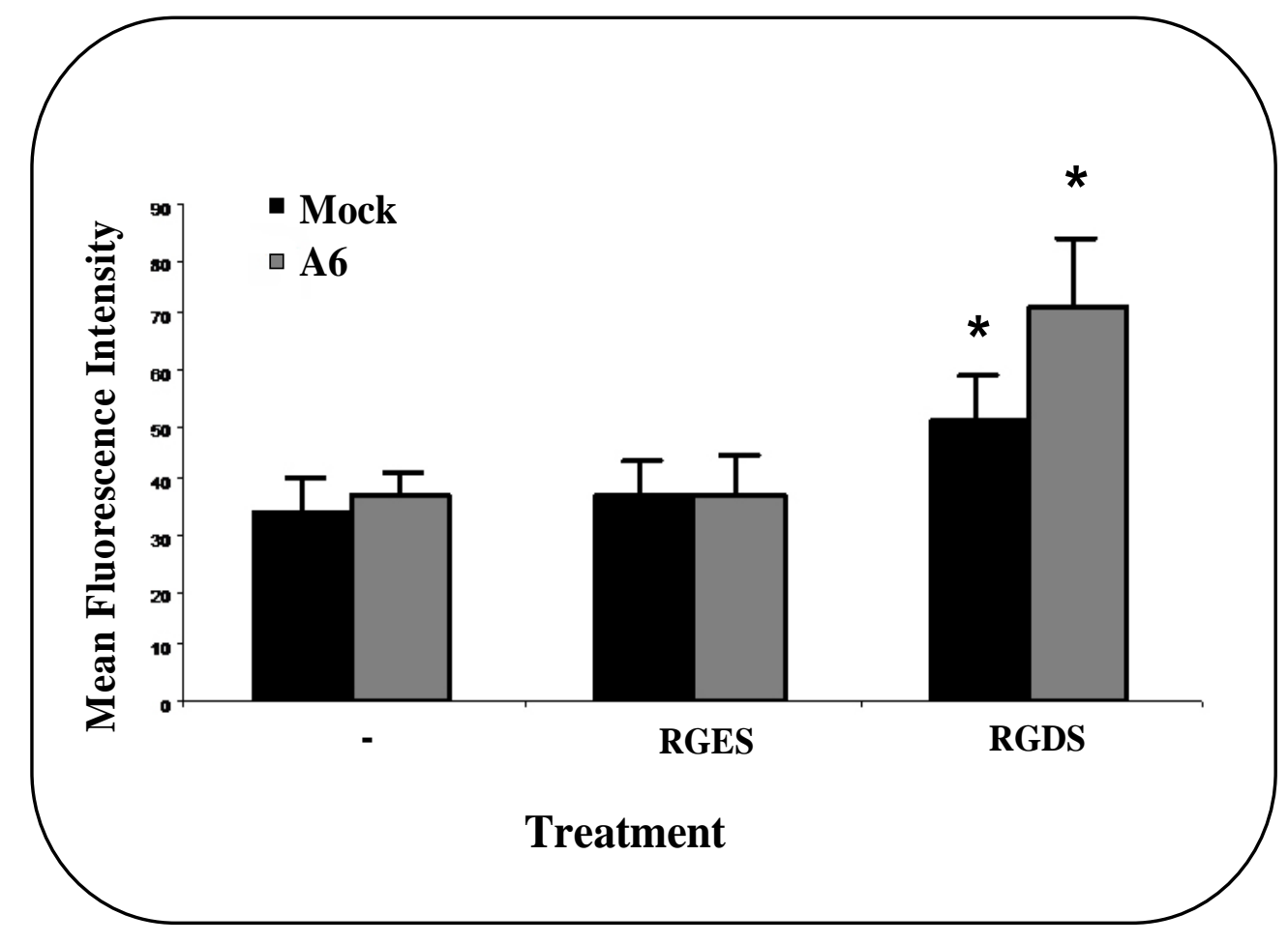

Figure 4.28 FCM Analysis of B44 Antibody Binding to Mock and A6 Cells

Results of quantitative Flow cytometry (FCM) experiments comparing relative binding of $\beta 1$ conformation sensitive antibody (B44) to Mock and A6 cells in the presence of integrin ligand-mimetic peptide RGDS or the negative control peptide RGES. * indicates $\mathrm{p}<0.05$ between RGDS treated Mock and A6 cells. The error bars represent the standard deviation. 


\section{Chapter 5: Summary}

In the current study we investigated the mechanisms governing CD9 effects on cell migration. We examined the contribution of the signaling pathways, PI-3K, PKC, and MAPK that were previously shown to be important in regulating cell migration. CD9 induced haptotactic cell migration on FN was inhibited by inhibitors of the PI-3K pathway but not by the inhibitors of PKC or MAPK pathway. Downregulation of PI-3K via dominant/negative PI-3K cDNA transfection also inhibited CD9 promoted haptotactic CHO cell migration. In support of these findings, both stable and transient expression of CD9 in CHO cells and RASM cells, respectively, induced enhanced phosphorylation of serine 473 on Akt in response to FN. PI-3K inhibitors abolished CD9 effects on Akt phosphorylation under these conditions demonstrating that Akt phosphorylation was reflective of PI-3K activity.

On adhesion to FN, CD9-CHO cells exhibited enhanced tyrosine phosphorylation of protein in the $105-250 \mathrm{kDa}$ molecular weight range which was identified as the signaling adaptor protein p130Cas. Subsequent examination revealed that total p130Cas protein were significantly higher in CD9-CHO cells compared to Mock control cells. The putative role of p130Cas in CD9 mediated cell migration was investigated via p130Cas specific siRNA mediated knockdown. Inhibition of p130Cas expression levels did not significantly affect haptotactic migration of CD9-CHO cells on FN.

Using Brij-98 as the detergent to solubilize membrane proteins, we investigated the interactions of CD9 with $\beta 1$ and $\beta 3$ integrins by IP. In platelets, CD9 physically associated with the integrin GPIIb/IIIa and in cultured cells CD9 associated with the $\beta 1$ 
integrin. In RASM cells, upon transient expression of CD9, integrin $\alpha 5 \beta 1$ was found in complex with CD9 and in MEF, endogenously expressed CD9 associated with $\alpha 5 \beta 1$.

CD9 expressing $\mathrm{CHO}$ cells retained their promigratory phenotype after serum starvation demonstrating that CD9 effects on cell migration are predominately governed by cell adhesion mechanisms rather than those involving growth factor receptors. The observation that the $\alpha 5 \beta 1$ blocking mAb, PB1 negated CD9 induced promigratory phenomenon provided further evidence that crosstalk between CD9 and $\alpha 5 \beta 1$ was required for $\mathrm{CD} 9$ promoted migratory activity in response to $\mathrm{FN}$. In $\mathrm{CHO}$ cells, CD9 expression significantly enhanced the binding of a conformation sensitive mAb to activated $\beta 1$ in the presence of $\alpha 5 \beta 1$ activating peptide. These data indicated that CD9 may stabilize the active conformation of integrin $\alpha 5 \beta 1$.

The mechanism by which CD9 enhances PI-3K activity in cells still remains to be elucidated. Previous studies showed that CD9 immune complexes were demonstrated to contain PI-4K activity (20). However, an association of CD9 with PI-3K activity has not been reported. In our observation, immunoprecipitation of CD9 from $\mathrm{CHO}$ cells did not reveal the presence of the PI-3K subunits (data not shown) suggesting that there is no direct physical interaction of PI-3K with CD9. We hypothesize that CD9 mediated activation of integrin $\beta 1$ is required for PI-3K activation.

Both FAK and Src are tyrosine kinases that are known to be tyrosine phosphorylated upon integrin interaction with ECM leading to activation of PI-3K. Our preliminary finding suggest that phosphorylation of FAK on Y397 is not significantly altered upon CD9 expression (data not shown). Phosphorylation of FAK on Y397 residue was shown to be important in regulating PI-3K activity (266). Expression of CD9 also 
did not significantly modify the phosphorylation state of Src and Src specific inhibitor SU6656 did not inhibit CD9 promoted cell migration (data not shown). These findings indicate that CD9 mediated activation of PI-3K does not require FAK and Src tyrosine kinases. In some model systems, Integrin $\beta 1$ mediated activation of PI-3K was shown occur independent of FAK, Src activation (273).

$\beta 1$ transmembrane domain was shown to be important in the activation of PI-3K pathway and mutations in the $\beta 1$ transmembrane were shown to adversely affect $\beta 1$ mediated signaling pathway. Specifically, $\beta 1$ transmembrane domain mutations significantly altered p130Cas phosphorylation that was FAK independent but was inhibited by PI-3K inhibitor LY294002 (274). These results suggest that p130Cas may act downstream of PI-3K (274). Since only low efficiency siRNA transfection methods were used to downregulate p130Cas to study the effects on cell motility, the precise role of p130Cas in CD9 mediated migration and PI-3K activation further needs to be studied by more efficient methods for transfection.

Cytoplasmic tail of $\beta 1$ interacts with intracellular proteins such as talin and $\alpha$ actinin that link the integrin to the cytoskeleton (275). In migrating cells focal adhesion formation and turnover is determined by the extent of association between integrin and the cytoskeleton. Studying the roles of talin, $\alpha$-actinin and characterizing the focal adhesion may further reveal the link between CD9 and PI-3K activation. The final common pathway leading to adhesive properties of cells such as cell motility and spreading involve changes in the cytoskeletal architecture. Since CD9 expressing cells exhibit characteristic adhesive phenotypes, we speculate that activation of PI-3K leads to changes in the cellular cytoskeleton. 
Overwhelming evidence suggest that PI-3K is directly involved in regulating cytoskeletal architecture. Activation of PI-3K leads to generation phosphoinositides products that serve as docking sites for the PH domain containing proteins (276). Phosphoinositide products and protein interacting with them were shown to modulate guanine nucleotide exchange (GEF) activity of certain cytosolic proteins that regulate Rho family of GTPases such as Rac (277).Ahdeison of cells to matrix proteins such as FN was also shown to promote Rac activity (278). In motile cells, Rac has been shown to be important in lamellipodia formation at the leading edge of the cell (279). Signaling molecules such as Akt that are activated downstream of PI-3K were also shown to regulate Rac activity (280). Based on these evidences, we speculate CD9 expression activates Rho family of GTPases downstream of PI-3K activation leading to enhanced cell motility. Figure 5.1 summarizes the CD9 mediated intracellular events that lead to the activation of PI-3K pathway and cell motility.

CD9 mediated cell motility has functional implications in several biologically important phenomenon such as vascular injury response. Our observations suggest that CD9 expression is altered VSMC after vascular injury and antibodies to CD9 modulate neointima formation in an injured vessel (Jennings et al, unpublished observations). These findings underscore an important regulatory role for CD9 in smooth muscle cells. In addition to vascular smooth muscle cells, CD9 is also expressed in endothelial cells. Since endothelial cells also participate in vascular injury response and because CD9 was shown to regulate endothelial cell migration we speculate that understanding the mechanism underlying CD9 mediated cell migration lay a foundation for the development of novel therapeutic strategies for cardiovascular diseases. 


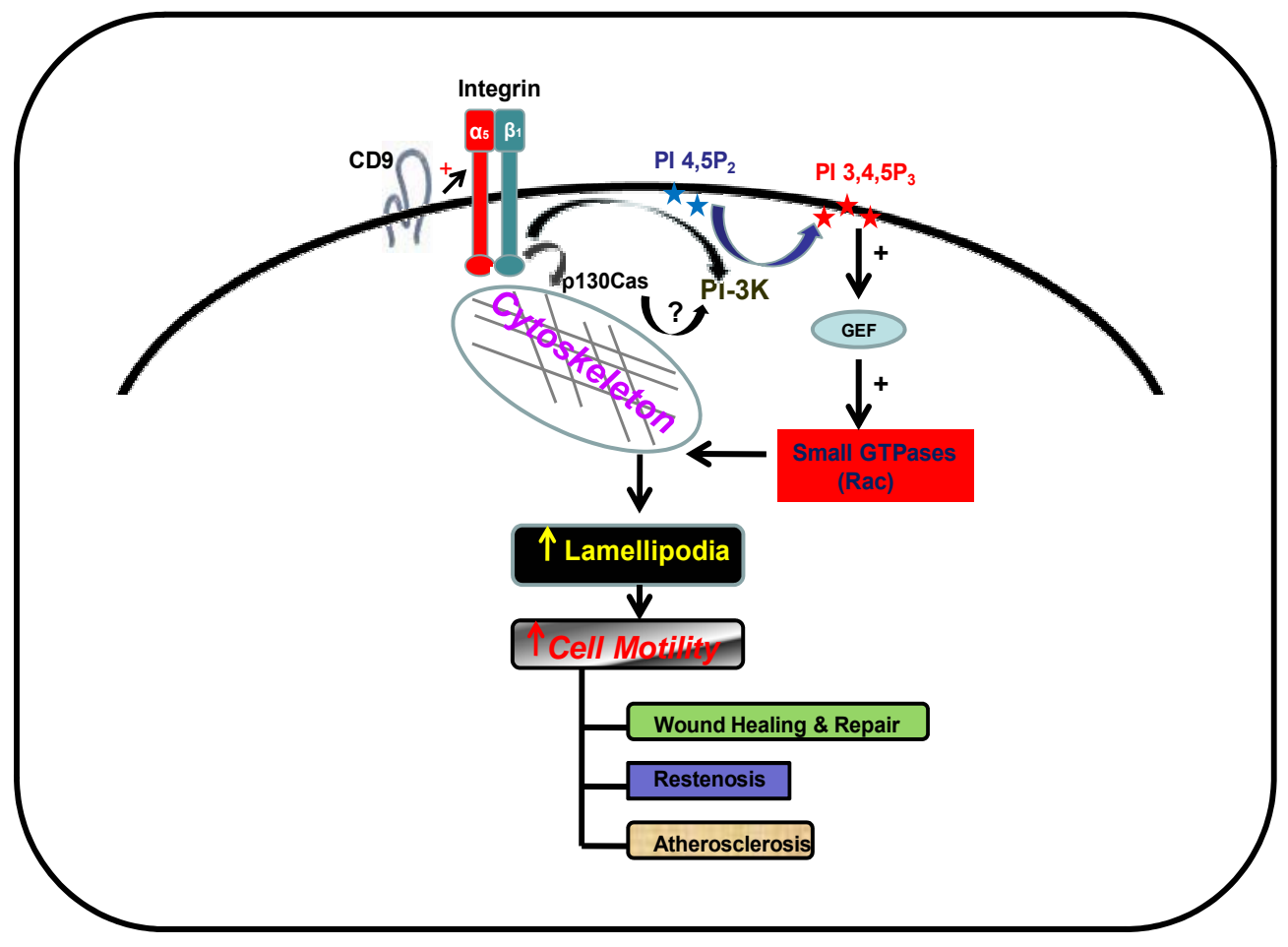

Figure 5.1 Summary of Events Leading to CD9 Mediated Cell Motility

CD9 can upregulate integrin $\alpha 5 \beta 1$ leading to activation of PI-3K. The phosphoinositides product (PI 3,4,5 P3) generated regulate the activity of the Rho family of GTPase, Rac. Enhanced Rac activity in cells can lead to lamellipodia formation and enhanced cell motility. 


\section{LIST OF REFERENCES}

1. Jennings, M. L. (1989) Topography of membrane proteins. Annu Rev Biochem 58, 999-1027.

2. Cramer, W. A., Engelman, D. M., Von Heijne, G., and Rees, D. C. (1992) Forces involved in the assembly and stabilization of membrane proteins. Faseb $J 6$, 3397-3402.

3. Boucheix, C., and Rubinstein, E. (2001) Tetraspanins. Cell Mol Life Sci 58, 11891205.

4. $\quad$ Fradkin, L. G., Kamphorst, J. T., DiAntonio, A., Goodman, C. S., and Noordermeer, J. N. (2002) Genomewide analysis of the Drosophila tetraspanins reveals a subset with similar function in the formation of the embryonic synapse. Proc Natl Acad Sci U S A 99, 13663-13668.

5. Moribe, H., Yochem, J., Yamada, H., Tabuse, Y., Fujimoto, T., and Mekada, E. (2004) Tetraspanin protein (TSP-15) is required for epidermal integrity in Caenorhabditis elegans. J Cell Sci 117, 5209-5220.

6. Tran, M. H., Pearson, M. S., Bethony, J. M., Smyth, D. J., Jones, M. K., Duke, M., Don, T. A., McManus, D. P., Correa-Oliveira, R., and Loukas, A. (2006)

Tetraspanins on the surface of Schistosoma mansoni are protective antigens against schistosomiasis. Nat Med 12, 835-840.

7. Clergeot, P. H., Gourgues, M., Cots, J., Laurans, F., Latorse, M. P., Pepin, R., Tharreau, D., Notteghem, J. L., and Lebrun, M. H. (2001) PLS1, a gene encoding a tetraspanin-like protein, is required for penetration of rice leaf by the fungal pathogen Magnaporthe grisea. Proc Natl Acad Sci U S A 98, 6963-6968.

8. Hemler, M. E. (2001) Specific tetraspanin functions. J Cell Biol 155, 1103-1107.

9. Rubinstein, E., Boucheix, C (1999) Tetraspans. In Guidebook to the Extracellular Matrix and Adhesion Proteins (Kreis, T., Vale, R, ed) pp. 321-324, Oxford University Press, Oxford.

10. Crosbie, R. H., Heighway, J., Venzke, D. P., Lee, J. C., and Campbell, K. P. (1997) Sarcospan, the 25-kDa transmembrane component of the dystrophinglycoprotein complex. J Biol Chem 272, 31221-31224.

11. Ishibashi, K., Suzuki, M., Sasaki, S., and Imai, M. (2001) Identification of a new multigene four-transmembrane family (MS4A) related to CD20, HTm4 and beta subunit of the high-affinity IgE receptor. Gene 264, 87-93.

12. Kitadokoro, K., Bordo, D., Galli, G., Petracca, R., Falugi, F., Abrignani, S., Grandi, G., and Bolognesi, M. (2001) CD81 extracellular domain 3D structure: insight into the tetraspanin superfamily structural motifs. Embo J 20, 12-18.

13. Kitadokoro, K., Galli, G., Petracca, R., Falugi, F., Grandi, G., and Bolognesi, M. (2001) Crystallization and preliminary crystallographic studies on the large extracellular domain of human $\mathrm{CD} 81$, a tetraspanin receptor for hepatitis $\mathrm{C}$ virus. Acta Crystallogr D Biol Crystallogr 57, 156-158.

14. Berditchevski, F., and Odintsova, E. (2007) Tetraspanins as regulators of protein trafficking. Traffic 8, 89-96. 
15. He, B., Liu, L., Cook, G. A., Grgurevich, S., Jennings, L. K., and Zhang, X. A. (2005) Tetraspanin CD82 attenuates cellular morphogenesis through downregulating integrin alpha6-mediated cell adhesion. J Biol Chem 280, 3346-3354.

16. Kovalenko, O. V., Metcalf, D. G., DeGrado, W. F., and Hemler, M. E. (2005) Structural organization and interactions of transmembrane domains in tetraspanin proteins. BMC Struct Biol 5, 11.

17. Tu, L., Kong, X. P., Sun, T. T., and Kreibich, G. (2006) Integrity of all four transmembrane domains of the tetraspanin uroplakin $\mathrm{Ib}$ is required for its exit from the ER. J Cell Sci 119, 5077-5086.

18. Hemler, M. E. (2003) Tetraspanin proteins mediate cellular penetration, invasion, and fusion events and define a novel type of membrane microdomain. Annu Rev Cell Dev Biol 19, 397-422.

19. Stipp, C. S., and Hemler, M. E. (2000) Transmembrane-4-superfamily proteins CD151 and CD81 associate with alpha 3 beta 1 integrin, and selectively contribute to alpha 3 beta 1-dependent neurite outgrowth. J Cell Sci 113 ( Pt 11), 1871-1882.

20. Yauch, R. L., and Hemler, M. E. (2000) Specific interactions among transmembrane 4 superfamily (TM4SF) proteins and phosphoinositide 4-kinase. Biochem J 351 Pt 3, 629-637.

21. Tu, L., Sun, T. T., and Kreibich, G. (2002) Specific heterodimer formation is a prerequisite for uroplakins to exit from the endoplasmic reticulum. Mol Biol Cell $13,4221-4230$.

22. Serru, V., Le Naour, F., Billard, M., Azorsa, D. O., Lanza, F., Boucheix, C., and Rubinstein, E. (1999) Selective tetraspan-integrin complexes (CD81/alpha4beta1, CD151/alpha3beta1, CD151/alpha6beta1) under conditions disrupting tetraspan interactions. Biochem J 340 ( Pt 1), 103-111.

23. Charrin, S., Manie, S., Oualid, M., Billard, M., Boucheix, C., and Rubinstein, E. (2002) Differential stability of tetraspanin/tetraspanin interactions: role of palmitoylation. FEBS Lett 516, 139-144.

24. Arnaout, M. A., Mahalingam, B., and Xiong, J. P. (2005) Integrin structure, allostery, and bidirectional signaling. Annu Rev Cell Dev Biol 21, 381-410.

25. Humphries, J. D., Byron, A., and Humphries, M. J. (2006) Integrin ligands at a glance. J Cell Sci 119, 3901-3903.

26. Tuckwell, D. S., Weston, S. A., and Humphries, M. J. (1993) Integrins: a review of their structure and mechanisms of ligand binding. Symp Soc Exp Biol 47, $107-$ 136.

27. Fitter, S., Sincock, P. M., Jolliffe, C. N., and Ashman, L. K. (1999) Transmembrane 4 superfamily protein CD151 (PETA-3) associates with beta 1 and alpha IIb beta 3 integrins in haemopoietic cell lines and modulates cell-cell adhesion. Biochem J 338 ( Pt 1), 61-70.

28. Shaw, A. R., Domanska, A., Mak, A., Gilchrist, A., Dobler, K., Visser, L., Poppema, S., Fliegel, L., Letarte, M., and Willett, B. J. (1995) Ectopic expression of human and feline CD9 in a human B cell line confers beta 1 integrin-dependent motility on fibronectin and laminin substrates and enhanced tyrosine phosphorylation. J Biol Chem 270, 24092-24099. 
29. Stipp, C. S., Kolesnikova, T. V., and Hemler, M. E. (2001) EWI-2 is a major CD9 and CD81 partner and member of a novel Ig protein subfamily. $J$ Biol Chem 276, 40545-40554.

30. Stipp, C. S., Kolesnikova, T. V., and Hemler, M. E. (2003) EWI-2 regulates alpha3beta1 integrin-dependent cell functions on laminin-5. J Cell Biol 163, $1167-1177$.

31. Imai, T., Kakizaki, M., Nishimura, M., and Yoshie, O. (1995) Molecular analyses of the association of CD4 with two members of the transmembrane 4 superfamily, CD81 and CD82. J Immunol 155, 1229-1239.

32. Le Naour, F., Charrin, S., Labas, V., Le Caer, J. P., Boucheix, C., and Rubinstein, E. (2004) Tetraspanins connect several types of Ig proteins: IgM is a novel component of the tetraspanin web on B-lymphoid cells. Cancer Immunol Immunother 53, 148-152.

33. Higashiyama, S., Iwamoto, R., Goishi, K., Raab, G., Taniguchi, N., Klagsbrun, M., and Mekada, E. (1995) The membrane protein CD9/DRAP 27 potentiates the juxtacrine growth factor activity of the membrane-anchored heparin-binding EGF-like growth factor. J Cell Biol 128, 929-938.

34. Mitamura, T., Iwamoto, R., Umata, T., Yomo, T., Urabe, I., Tsuneoka, M., and Mekada, E. (1992) The 27-kD diphtheria toxin receptor-associated protein (DRAP27) from vero cells is the monkey homologue of human CD9 antigen: expression of DRAP27 elevates the number of diphtheria toxin receptors on toxin-sensitive cells. J Cell Biol 118, 1389-1399.

35. Toledo, M. S., Suzuki, E., Handa, K., and Hakomori, S. (2005) Effect of ganglioside and tetraspanins in microdomains on interaction of integrins with fibroblast growth factor receptor. J Biol Chem 280, 16227-16234.

36. Berditchevski, F., Tolias, K. F., Wong, K., Carpenter, C. L., and Hemler, M. E. (1997) A novel link between integrins, transmembrane-4 superfamily proteins (CD63 and CD81), and phosphatidylinositol 4-kinase. J Biol Chem 272, 25952598.

37. Yauch, R. L., Berditchevski, F., Harler, M. B., Reichner, J., and Hemler, M. E. (1998) Highly stoichiometric, stable, and specific association of integrin alpha3beta1 with CD151 provides a major link to phosphatidylinositol 4-kinase, and may regulate cell migration. Mol Biol Cell 9, 2751-2765.

38. Zhang, X. A., Bontrager, A. L., and Hemler, M. E. (2001) Transmembrane-4 superfamily proteins associate with activated protein kinase $\mathrm{C}(\mathrm{PKC})$ and link PKC to specific beta(1) integrins. J Biol Chem 276, 25005-25013.

39. Zhang, X. A., He, B., Zhou, B., and Liu, L. (2003) Requirement of the p130CASCrk coupling for metastasis suppressor KAI1/CD82-mediated inhibition of cell migration. J Biol Chem 278, 27319-27328.

40. Pileri, P., Uematsu, Y., Campagnoli, S., Galli, G., Falugi, F., Petracca, R., Weiner, A. J., Houghton, M., Rosa, D., Grandi, G., and Abrignani, S. (1998) Binding of hepatitis C virus to CD81. Science 282, 938-941.

41. Petracca, R., Falugi, F., Galli, G., Norais, N., Rosa, D., Campagnoli, S., Burgio, V., Di Stasio, E., Giardina, B., Houghton, M., Abrignani, S., and Grandi, G. (2000) Structure-function analysis of hepatitis C virus envelope-CD81 binding. $J$ Virol 74, 4824-4830. 
42. Sasaki, M., Yamauchi, K., Nakanishi, T., Kamogawa, Y., and Hayashi, N. (2003) In vitro binding of hepatitis $\mathrm{C}$ virus to CD81-positive and -negative human cell lines. J Gastroenterol Hepatol 18, 74-79.

43. Silvie, O., Rubinstein, E., Franetich, J. F., Prenant, M., Belnoue, E., Renia, L., Hannoun, L., Eling, W., Levy, S., Boucheix, C., and Mazier, D. (2003)

Hepatocyte CD81 is required for Plasmodium falciparum and Plasmodium yoelii sporozoite infectivity. Nat Med 9, 93-96.

44. Fukudome, K., Furuse, M., Imai, T., Nishimura, M., Takagi, S., Hinuma, Y., and Yoshie, O. (1992) Identification of membrane antigen C33 recognized by monoclonal antibodies inhibitory to human T-cell leukemia virus type 1 (HTLV1)-induced syncytium formation: altered glycosylation of C33 antigen in HTLV1-positive T cells. $J$ Virol 66, 1394-1401.

45. Imai, T., Fukudome, K., Takagi, S., Nagira, M., Furuse, M., Fukuhara, N., Nishimura, M., Hinuma, Y., and Yoshie, O. (1992) C33 antigen recognized by monoclonal antibodies inhibitory to human $\mathrm{T}$ cell leukemia virus type 1-induced syncytium formation is a member of a new family of transmembrane proteins including CD9, CD37, CD53, and CD63. J Immunol 149, 2879-2886.

46. von Lindern, J. J., Rojo, D., Grovit-Ferbas, K., Yeramian, C., Deng, C., Herbein, G., Ferguson, M. R., Pappas, T. C., Decker, J. M., Singh, A., Collman, R. G., and O'Brien, W. A. (2003) Potential role for CD63 in CCR5-mediated human immunodeficiency virus type 1 infection of macrophages. J Virol 77, 3624-3633.

47. Gordon-Alonso, M., Yanez-Mo, M., Barreiro, O., Alvarez, S., Munoz-Fernandez, M. A., Valenzuela-Fernandez, A., and Sanchez-Madrid, F. (2006) Tetraspanins CD9 and CD81 modulate HIV-1-induced membrane fusion. J Immunol 177, 5129-5137.

48. Engering, A., and Pieters, J. (2001) Association of distinct tetraspanins with MHC class II molecules at different subcellular locations in human immature dendritic cells. Int Immunol 13, 127-134.

49. Tarrant, J. M., Robb, L., van Spriel, A. B., and Wright, M. D. (2003) Tetraspanins: molecular organisers of the leukocyte surface. Trends Immunol 24, 610-617.

50. Ono, Y., Fukuhara, N., and Yoshie, O. (1997) Transcriptional activity of TAL1 in $\mathrm{T}$ cell acute lymphoblastic leukemia (T-ALL) requires RBTN1 or -2 and induces TALLA1, a highly specific tumor marker of T-ALL. J Biol Chem 272, 45764581.

51. Kersey, J. H., LeBien, T. W., Abramson, C. S., Newman, R., Sutherland, R., and Greaves, M. (1981) P-24: a human leukemia-associated and lymphohemopoietic progenitor cell surface structure identified with monoclonal antibody. J Exp Med $153,726-731$.

52. Kubista, B., Erovic, B. M., Klinger, H., Sulzbacher, I., and Trieb, K. (2004) CD9 expression is not a prognostic factor in human osteosarcoma. Cancer Lett 209, 105-110.

53. Liu, W. M., and Zhang, X. A. (2006) KAI1/CD82, a tumor metastasis suppressor. Cancer Lett 240, 183-194. 
54. Kopczynski, C. C., Davis, G. W., and Goodman, C. S. (1996) A neural tetraspanin, encoded by late bloomer, that facilitates synapse formation. Science 271, 1867-1870.

55. Zemni, R., Bienvenu, T., Vinet, M. C., Sefiani, A., Carrie, A., Billuart, P., McDonell, N., Couvert, P., Francis, F., Chafey, P., Fauchereau, F., Friocourt, G., des Portes, V., Cardona, A., Frints, S., Meindl, A., Brandau, O., Ronce, N., Moraine, C., van Bokhoven, H., Ropers, H. H., Sudbrak, R., Kahn, A., Fryns, J. P., Beldjord, C., and Chelly, J. (2000) A new gene involved in X-linked mental retardation identified by analysis of an X;2 balanced translocation. Nat Genet 24, 167-170.

56. Hosokawa, Y., Ueyama, E., Morikawa, Y., Maeda, Y., Seto, M., and Senba, E. (1999) Molecular cloning of a cDNA encoding mouse A15, a member of the transmembrane 4 superfamily, and its preferential expression in brain neurons. Neurosci Res 35, 281-290.

57. Kaji, K., Oda, S., Shikano, T., Ohnuki, T., Uematsu, Y., Sakagami, J., Tada, N., Miyazaki, S., and Kudo, A. (2000) The gamete fusion process is defective in eggs of Cd9-deficient mice. Nat Genet 24, 279-282.

58. Le Naour, F., Rubinstein, E., Jasmin, C., Prenant, M., and Boucheix, C. (2000) Severely reduced female fertility in CD9-deficient mice. Science 287, 319-321.

59. Miyado, K., Yamada, G., Yamada, S., Hasuwa, H., Nakamura, Y., Ryu, F., Suzuki, K., Kosai, K., Inoue, K., Ogura, A., Okabe, M., and Mekada, E. (2000) Requirement of CD9 on the egg plasma membrane for fertilization. Science 287, 321-324.

60. Robb, L., Tarrant, J., Groom, J., Ibrahim, M., Li, R., Borobakas, B., and Wright, M. D. (2001) Molecular characterisation of mouse and human TSSC6: evidence that TSSC6 is a genuine member of the tetraspanin superfamily and is expressed specifically in haematopoietic organs. Biochim Biophys Acta 1522, 31-41.

61. Tarrant, J. M., Groom, J., Metcalf, D., Li, R., Borobokas, B., Wright, M. D., Tarlinton, D., and Robb, L. (2002) The absence of Tssc6, a member of the tetraspanin superfamily, does not affect lymphoid development but enhances in vitro T-cell proliferative responses. Mol Cell Biol 22, 5006-5018.

62. Goschnick, M. W., Lau, L. M., Wee, J. L., Liu, Y. S., Hogarth, P. M., Robb, L. M., Hickey, M. J., Wright, M. D., and Jackson, D. E. (2006) Impaired "outsidein" integrin alphaIIbbeta3 signaling and thrombus stability in TSSC6-deficient mice. Blood 108, 1911-1918.

63. Moore, K., Cooper, S. A., and Jones, D. B. (1987) Use of the monoclonal antibody WR17, identifying the CD37 gp40-45 Kd antigen complex, in the diagnosis of B-lymphoid malignancy. J Pathol 152, 13-21.

64. Knobeloch, K. P., Wright, M. D., Ochsenbein, A. F., Liesenfeld, O., Lohler, J., Zinkernagel, R. M., Horak, I., and Orinska, Z. (2000) Targeted inactivation of the tetraspanin CD37 impairs T-cell-dependent B-cell response under suboptimal costimulatory conditions. Mol Cell Biol 20, 5363-5369.

65. Shoham, T., Rajapaksa, R., Boucheix, C., Rubinstein, E., Poe, J. C., Tedder, T. F., and Levy, S. (2003) The tetraspanin CD81 regulates the expression of CD19 during B cell development in a postendoplasmic reticulum compartment. $J$ Immunol 171, 4062-4072. 
66. Levy, S., Todd, S. C., and Maecker, H. T. (1998) CD81 (TAPA-1): a molecule involved in signal transduction and cell adhesion in the immune system. Annu Rev Immunol 16, 89-109.

67. Rubinstein, E., Ziyyat, A., Prenant, M., Wrobel, E., Wolf, J. P., Levy, S., Le Naour, F., and Boucheix, C. (2006) Reduced fertility of female mice lacking CD81. Dev Biol 290, 351-358.

68. Wright, M. D., Geary, S. M., Fitter, S., Moseley, G. W., Lau, L. M., Sheng, K. C., Apostolopoulos, V., Stanley, E. G., Jackson, D. E., and Ashman, L. K. (2004) Characterization of mice lacking the tetraspanin superfamily member CD151. Mol Cell Biol 24, 5978-5988.

69. Lau, L. M., Wee, J. L., Wright, M. D., Moseley, G. W., Hogarth, P. M., Ashman, L. K., and Jackson, D. E. (2004) The tetraspanin superfamily member CD151 regulates outside-in integrin alphaIIbbeta3 signaling and platelet function. Blood 104, 2368-2375.

70. Sachs, N., Kreft, M., van den Bergh Weerman, M. A., Beynon, A. J., Peters, T. A., Weening, J. J., and Sonnenberg, A. (2006) Kidney failure in mice lacking the tetraspanin CD151. J Cell Biol 175, 33-39.

71. Brooks, D. A., Bradley, J., and Zola, H. (1982) A differentiation antigen expressed selectively by a proportion of human blood cells: detection with a monoclonal antibody. Pathology 14, 5-11.

72. Deng, C. T., Terasaki, P. I., Iwaki, Y., Hofman, F. M., Koeffler, P., Cahan, L., El Awar, N., and Billing, R. (1983) A monoclonal antibody cross-reactive with human platelets, megakaryocytes, and common acute lymphocytic leukemia cells. Blood 61, 759-764.

73. Jones, N. H., Borowitz, M. J., and Metzgar, R. S. (1982) Characterization and distribution of a 24,000-molecular weight antigen defined by a monoclonal antibody (DU-ALL-1) elicited to common acute lymphoblastic leukemia (cALL) cells. Leuk Res 6, 449-464.

74. Komada, Y., Peiper, S. C., Melvin, S. L., Metzger, D. W., Tarnowski, B. H., and Green, A. A. (1983) A monoclonal antibody (SJ-9A4) to P24 present on common alls, neuroblastomas and platelets - I. Characterization and development of a unique radioimmunometric assay. Leuk Res 7, 487-498.

75. Bernard, A., and Boumsell, L. (1984) The clusters of differentiation (CD) defined by the First International Workshop on Human Leucocyte Differentiation Antigens. Hum Immunol 11, 1-10.

76. Benoit, P., Gross, M. S., Frachet, P., Frezal, J., Uzan, G., Boucheix, C., and Nguyen, V. C. (1991) Assignment of the human CD9 gene to chromosome 12 (region P13) by use of human specific DNA probes. Hum Genet 86, 268-272.

77. Lanza, F., Wolf, D., Fox, C. F., Kieffer, N., Seyer, J. M., Fried, V. A., Coughlin, S. R., Phillips, D. R., and Jennings, L. K. (1991) cDNA cloning and expression of platelet $\mathrm{p} 24 / \mathrm{CD} 9$. Evidence for a new family of multiple membrane-spanning proteins. J Biol Chem 266, 10638-10645.

78. Paulson, J. C. (1989) Glycoproteins: what are the sugar chains for? Trends Biochem Sci 14, 272-276.

79. Ono, M., Handa, K., Withers, D. A., and Hakomori, S. (2000) Glycosylation effect on membrane domain (GEM) involved in cell adhesion and motility: a 
preliminary note on functional alpha3, alpha5-CD82 glycosylation complex in ldlD 14 cells. Biochem Biophys Res Commun 279, 744-750.

80. Martin, F., Roth, D. M., Jans, D. A., Pouton, C. W., Partridge, L. J., Monk, P. N., and Moseley, G. W. (2005) Tetraspanins in viral infections: a fundamental role in viral biology? J Virol 79, 10839-10851.

81. Dietrich, L. E., and Ungermann, C. (2004) On the mechanism of protein palmitoylation. EMBO Rep 5, 1053-1057.

82. Yang, X., Kovalenko, O. V., Tang, W., Claas, C., Stipp, C. S., and Hemler, M. E. (2004) Palmitoylation supports assembly and function of integrin-tetraspanin complexes. J Cell Biol 167, 1231-1240.

83. Boucheix, C., and Benoit, P. (1988) CD9 antigen: will platelet physiology help to explain the function of a surface molecule during hemopoietic differentiation? Nouv Rev Fr Hematol 30, 201-202.

84. Brisson, C., Azorsa, D. O., Jennings, L. K., Moog, S., Cazenave, J. P., and Lanza, F. (1997) Co-localization of CD9 and GPIIb-IIIa (alpha IIb beta 3 integrin) on activated platelet pseudopods and alpha-granule membranes. Histochem J 29, 153-165.

85. Jennings, L. K., Crossno, J. T., Jr., Fox, C. F., White, M. M., and Green, C. A. (1994) Platelet p24/CD9, a member of the tetraspanin family of proteins. Ann N Y Acad Sci 714, 175-184.

86. Wang, X. Q., Evans, G. F., Alfaro, M. L., and Zuckerman, S. H. (2002) Downregulation of macrophage CD9 expression by interferon-gamma. Biochem Biophys Res Commun 290, 891-897.

87. Fernvik, E., Hallden, G., Hed, J., and Lundahl, J. (1995) Intracellular and surface distribution of CD9 in human eosinophils. Apmis 103, 699-706.

88. Fureder, W., Agis, H., Sperr, W. R., Lechner, K., and Valent, P. (1994) The surface membrane antigen phenotype of human blood basophils. Allergy 49, 861865.

89. Won, W. J., and Kearney, J. F. (2002) CD9 is a unique marker for marginal zone B cells, B1 cells, and plasma cells in mice. J Immunol 168, 5605-5611.

90. Barreiro, O., Yanez-Mo, M., Sala-Valdes, M., Gutierrez-Lopez, M. D., Ovalle, S., Higginbottom, A., Monk, P. N., Cabanas, C., and Sanchez-Madrid, F. (2005) Endothelial tetraspanin microdomains regulate leukocyte firm adhesion during extravasation. Blood 105, 2852-2861.

91. Klein-Soyer, C., Azorsa, D. O., Cazenave, J. P., and Lanza, F. (2000) CD9 participates in endothelial cell migration during in vitro wound repair. Arterioscler Thromb Vasc Biol 20, 360-369.

92. Scherberich, A., Giannone, G., Perennou, E., Takeda, K., Boucheix, C., Rubinstein, E., Lanza, F., and Beretz, A. (2002) FAK-mediated inhibition of vascular smooth muscle cell migration by the tetraspanin CD9. Thromb Haemost 87, 1043-1050.

93. Scherberich, A., Moog, S., Haan-Archipoff, G., Azorsa, D. O., Lanza, F., and Beretz, A. (1998) Tetraspanin CD9 is associated with very late-acting integrins in human vascular smooth muscle cells and modulates collagen matrix reorganization. Arterioscler Thromb Vasc Biol 18, 1691-1697. 
94. Banerjee, S. A., Hadjiargyrou, M., and Patterson, P. H. (1997) An antibody to the tetraspan membrane protein CD9 promotes neurite formation in a partially alpha3beta1 integrin-dependent manner. J Neurosci 17, 2756-2765.

95. Schmidt, C., Kunemund, V., Wintergerst, E. S., Schmitz, B., and Schachner, M. (1996) CD9 of mouse brain is implicated in neurite outgrowth and cell migration in vitro and is associated with the alpha 6/beta 1 integrin and the neural adhesion molecule L1. J Neurosci Res 43, 12-31.

96. Anton, E. S., Hadjiargyrou, M., Patterson, P. H., and Matthew, W. D. (1995) CD9 plays a role in Schwann cell migration in vitro. J Neurosci 15, 584-595.

97. Ziyyat, A., Rubinstein, E., Monier-Gavelle, F., Barraud, V., Kulski, O., Prenant, M., Boucheix, C., Bomsel, M., and Wolf, J. P. (2006) CD9 controls the formation of clusters that contain tetraspanins and the integrin alpha 6 beta 1, which are involved in human and mouse gamete fusion. J Cell Sci 119, 416-424.

98. Li, Y. H., Hou, Y., Ma, W., Yuan, J. X., Zhang, D., Sun, Q. Y., and Wang, W. H. (2004) Localization of CD9 in pig oocytes and its effects on sperm-egg interaction. Reproduction 127, 151-157.

99. Hirano, T., Higuchi, T., Ueda, M., Inoue, T., Kataoka, N., Maeda, M., Fujiwara, H., and Fujii, S. (1999) CD9 is expressed in extravillous trophoblasts in association with integrin alpha3 and integrin alpha5. Mol Hum Reprod 5, 162167.

100. Takao, Y., Fujiwara, H., Yamada, S., Hirano, T., Maeda, M., Fujii, S., and Ueda, M. (1999) CD9 is expressed on the cell surface of human granulosa cells and associated with integrin alpha6beta1. Mol Hum Reprod 5, 303-310.

101. Jones, P. H., Bishop, L. A., and Watt, F. M. (1996) Functional significance of CD9 association with beta 1 integrins in human epidermal keratinocytes. Cell Adhes Commun 4, 297-305.

102. Jennings, L. K., Fox, C. F., Kouns, W. C., McKay, C. P., Ballou, L. R., and Schultz, H. E. (1990) The activation of human platelets mediated by anti-human platelet p24/CD9 monoclonal antibodies. J Biol Chem 265, 3815-3822.

103. Rendu, F., Boucheix, C., Lebret, M., Bourdeau, N., Benoit, P., Maclouf, J., Soria, C., and Levy-Toledano, S. (1987) Mechanisms of the mAb ALB6(CD9) induced human platelet activation: comparison with thrombin. Biochem Biophys Res Commun 146, 1397-1404.

104. Azorsa, D. O., Hyman, J. A., and Hildreth, J. E. (1991) CD63/Pltgp40: a platelet activation antigen identical to the stage-specific, melanoma-associated antigen ME491. Blood 78, 280-284.

105. Nishibori, M., Cham, B., McNicol, A., Shalev, A., Jain, N., and Gerrard, J. M. (1993) The protein CD63 is in platelet dense granules, is deficient in a patient with Hermansky-Pudlak syndrome, and appears identical to granulophysin. J Clin Invest 91, 1775-1782.

106. van der Zee, P. M., Biro, E., Ko, Y., de Winter, R. J., Hack, C. E., Sturk, A., and Nieuwland, R. (2006) P-selectin- and CD63-exposing platelet microparticles reflect platelet activation in peripheral arterial disease and myocardial infarction. Clin Chem 52, 657-664.

107. French, D. L., and Seligsohn, U. (2000) Platelet glycoprotein IIb/IIIa receptors and Glanzmann's thrombasthenia. Arterioscler Thromb Vasc Biol 20, 607-610. 
108. Slupsky, J. R., Seehafer, J. G., Tang, S. C., Masellis-Smith, A., and Shaw, A. R. (1989) Evidence that monoclonal antibodies against CD9 antigen induce specific association between CD9 and the platelet glycoprotein IIb-IIIa complex. J Biol Chem 264, 12289-12293.

109. Indig, F. E., Diaz-Gonzalez, F., and Ginsberg, M. H. (1997) Analysis of the tetraspanin CD9-integrin alphaIIbbeta3 (GPIIb-IIIa) complex in platelet membranes and transfected cells. Biochem J 327 (Pt 1), 291-298.

110. Longhurst, C. M., White, M. M., Wilkinson, D. A., and Jennings, L. K. (1999) A CD9, alphaIIbbeta3, integrin-associated protein, and GPIb/V/IX complex on the surface of human platelets is influenced by alphaIIbbeta3 conformational states. Eur J Biochem 263, 104-111.

111. Favier, R., Lecompte, T., Morel, M. C., Potevin, F., Benoit, P., Boucheix, C., Kaplan, C., and Samama, M. (1989) Calcium rise in human platelets elicited by anti-CD9 and -CD41 murine monoclonal antibodies. Thromb Res 55, 591-599.

112. Miyake, M., Nakano, K., Itoi, S. I., Koh, T., and Taki, T. (1996) Motility-related protein-1 (MRP-1/CD9) reduction as a factor of poor prognosis in breast cancer. Cancer Res 56, 1244-1249.

113. Higashiyama, M., Doi, O., Kodama, K., Yokouchi, H., Adachi, M., Huang, C. L., Taki, T., Kasugai, T., Ishiguro, S., Nakamori, S., and Miyake, M. (1997) Immunohistochemically detected expression of motility-related protein-1 (MRP$1 / \mathrm{CD} 9$ ) in lung adenocarcinoma and its relation to prognosis. Int J Cancer 74, 205-211.

114. Uchida, S., Shimada, Y., Watanabe, G., Li, Z. G., Hong, T., Miyake, M., and Imamura, M. (1999) Motility-related protein (MRP-1/CD9) and KAI1/CD82 expression inversely correlate with lymph node metastasis in oesophageal squamous cell carcinoma. Br J Cancer 79, 1168-1173.

115. Kusukawa, J., Ryu, F., Kameyama, T., and Mekada, E. (2001) Reduced expression of CD9 in oral squamous cell carcinoma: CD9 expression inversely related to high prevalence of lymph node metastasis. J Oral Pathol Med 30, 7379.

116. Funakoshi, T., Tachibana, I., Hoshida, Y., Kimura, H., Takeda, Y., Kijima, T., Nishino, K., Goto, H., Yoneda, T., Kumagai, T., Osaki, T., Hayashi, S., Aozasa, K., and Kawase, I. (2003) Expression of tetraspanins in human lung cancer cells: frequent downregulation of CD9 and its contribution to cell motility in small cell lung cancer. Oncogene 22, 674-687.

117. Cajot, J. F., Sordat, I., Silvestre, T., and Sordat, B. (1997) Differential display cloning identifies motility-related protein (MRP1/CD9) as highly expressed in primary compared to metastatic human colon carcinoma cells. Cancer Res 57, 2593-2597.

118. Sauer, G., Windisch, J., Kurzeder, C., Heilmann, V., Kreienberg, R., and Deissler, H. (2003) Progression of cervical carcinomas is associated with down-regulation of CD9 but strong local re-expression at sites of transendothelial invasion. Clin Cancer Res 9, 6426-6431.

119. Hori, H., Yano, S., Koufuji, K., Takeda, J., and Shirouzu, K. (2004) CD9 expression in gastric cancer and its significance. J Surg Res 117, 208-215. 
120. Masellis-Smith, A., Jensen, G. S., Seehafer, J. G., Slupsky, J. R., and Shaw, A. R. (1990) Anti-CD9 monoclonal antibodies induce homotypic adhesion of pre-B cell lines by a novel mechanism. J Immunol 144, 1607-1613.

121. Miyake, M., Koyama, M., Seno, M., and Ikeyama, S. (1991) Identification of the motility-related protein (MRP-1), recognized by monoclonal antibody M31-15, which inhibits cell motility. J Exp Med 174, 1347-1354.

122. Longo, N., Yanez-Mo, M., Mittelbrunn, M., de la Rosa, G., Munoz, M. L., Sanchez-Madrid, F., and Sanchez-Mateos, P. (2001) Regulatory role of tetraspanin CD9 in tumor-endothelial cell interaction during transendothelial invasion of melanoma cells. Blood 98, 3717-3726.

123. Mulrooney, J. P., Allen, J., Bickelhaupt, E., and Grabel, L. B. (2002) CD9alpha6beta1 interactions in migratory parietal endoderm cells. Cell Commun Adhes 9, 249-258.

124. Park, K. R., Inoue, T., Ueda, M., Hirano, T., Higuchi, T., Konishi, I., Fujiwara, H., and Fujii, S. (2000) Anti-CD9 monoclonal antibody-stimulated invasion of endometrial cancer cell lines in vitro: possible inhibitory effect of CD9 in endometrial cancer invasion. Mol Hum Reprod 6, 719-725.

125. Garcia-Lopez, M. A., Barreiro, O., Garcia-Diez, A., Sanchez-Madrid, F., and Penas, P. F. (2005) Role of tetraspanins CD9 and CD151 in primary melanocyte motility. J Invest Dermatol 125, 1001-1009.

126. Baudoux, B., Castanares-Zapatero, D., Leclercq-Smekens, M., Berna, N., and Poumay, Y. (2000) The tetraspanin CD9 associates with the integrin alpha6beta4 in cultured human epidermal keratinocytes and is involved in cell motility. Eur $J$ Cell Biol 79, 41-51.

127. Ikeyama, S., Koyama, M., Yamaoko, M., Sasada, R., and Miyake, M. (1993) Suppression of cell motility and metastasis by transfection with human motilityrelated protein (MRP-1/CD9) DNA. J Exp Med 177, 1231-1237.

128. Zheng, R., Yano, S., Zhang, H., Nakataki, E., Tachibana, I., Kawase, I., Hayashi, S., and Sone, S. (2005) CD9 overexpression suppressed the liver metastasis and malignant ascites via inhibition of proliferation and motility of small-cell lung cancer cells in NK cell-depleted SCID mice. Oncol Res 15, 365-372.

129. Longhurst, C. M., Jacobs, J. D., White, M. M., Crossno, J. T., Jr., Fitzgerald, D. A., Bao, J., Fitzgerald, T. J., Raghow, R., and Jennings, L. K. (2002) Chinese hamster ovary cell motility to fibronectin is modulated by the second extracellular loop of CD9. Identification of a putative fibronectin binding site. $J$ Biol Chem 277, 32445-32452.

130. Penas, P. F., Garcia-Lopez, M. A., and Barreiro del Rio, O. (2005) [Inhibition of the motility of melanoma cells using interference RNA against CD9]. Actas Dermosifiliogr 96, 30-36.

131. Chen, M. S., Almeida, E. A., Huovila, A. P., Takahashi, Y., Shaw, L. M., Mercurio, A. M., and White, J. M. (1999) Evidence that distinct states of the integrin alpha6beta1 interact with laminin and an ADAM. J Cell Biol 144, 549561.

132. Kaji, K., Oda, S., Miyazaki, S., and Kudo, A. (2002) Infertility of CD9-deficient mouse eggs is reversed by mouse CD9, human CD9, or mouse CD81; 
polyadenylated mRNA injection developed for molecular analysis of sperm-egg fusion. Dev Biol 247, 327-334.

133. Chen, M. S., Tung, K. S., Coonrod, S. A., Takahashi, Y., Bigler, D., Chang, A., Yamashita, Y., Kincade, P. W., Herr, J. C., and White, J. M. (1999) Role of the integrin-associated protein CD9 in binding between sperm ADAM 2 and the egg integrin alpha6beta1: implications for murine fertilization. Proc Natl Acad Sci U S A 96, 11830-11835.

134. Zhu, G. Z., Miller, B. J., Boucheix, C., Rubinstein, E., Liu, C. C., Hynes, R. O., Myles, D. G., and Primakoff, P. (2002) Residues SFQ (173-175) in the large extracellular loop of CD9 are required for gamete fusion. Development 129, 19952002.

135. Tachibana, I., and Hemler, M. E. (1999) Role of transmembrane 4 superfamily (TM4SF) proteins CD9 and CD81 in muscle cell fusion and myotube maintenance. J Cell Biol 146, 893-904.

136. Yi, T., Kim, H. J., Cho, J. Y., Woo, K. M., Ryoo, H. M., Kim, G. S., and Baek, J. H. (2006) Tetraspanin CD9 regulates osteoclastogenesis via regulation of $\mathrm{p} 44 / 42$ MAPK activity. Biochem Biophys Res Commun 347, 178-184.

137. Berditchevski, F. (2001) Complexes of tetraspanins with integrins: more than meets the eye. J Cell Sci 114, 4143-4151.

138. Rubinstein, E., Le Naour, F., Billard, M., Prenant, M., and Boucheix, C. (1994) CD9 antigen is an accessory subunit of the VLA integrin complexes. Eur $J$ Immunol 24, 3005-3013.

139. Kreidberg, J. A. (2000) Functions of alpha3beta1 integrin. Curr Opin Cell Biol $12,548-553$.

140. Okochi, H., Kato, M., Nashiro, K., Yoshie, O., Miyazono, K., and Furue, M. (1997) Expression of tetra-spans transmembrane family (CD9, CD37, CD53, CD63, CD81 and CD82) in normal and neoplastic human keratinocytes: an association of CD9 with alpha 3 beta 1 integrin. Br J Dermatol 137, 856-863.

141. Claas, C., Stipp, C. S., and Hemler, M. E. (2001) Evaluation of prototype transmembrane 4 superfamily protein complexes and their relation to lipid rafts. $J$ Biol Chem 276, 7974-7984.

142. Nakamura, Y., Handa, K., Iwamoto, R., Tsukamoto, T., Takahasi, M., and Mekada, E. (2001) Immunohistochemical distribution of CD9, heparin binding epidermal growth factor-like growth factor, and integrin alpha3beta1 in normal human tissues. J Histochem Cytochem 49, 439-444.

143. Kawakami, Y., Kawakami, K., Steelant, W. F., Ono, M., Baek, R. C., Handa, K., Withers, D. A., and Hakomori, S. (2002) Tetraspanin CD9 is a "proteolipid," and its interaction with alpha 3 integrin in microdomain is promoted by GM3 ganglioside, leading to inhibition of laminin-5-dependent cell motility. J Biol Chem 277, 34349-34358.

144. Dean, D. C., Iademarco, M. F., Rosen, G. D., and Sheppard, A. M. (1993) The integrin alpha 4 beta 1 and its counter receptor VCAM-1 in development and immune function. Am Rev Respir Dis 148, S43-46.

145. Postigo, A. A., Teixido, J., and Sanchez-Madrid, F. (1993) The alpha 4 beta 1/VCAM-1 adhesion pathway in physiology and disease. Res Immunol 144, $723-$ 735; discussion 754-762. 
146. Humphries, M. J., Sheridan, J., Mould, A. P., and Newham, P. (1995) Mechanisms of VCAM-1 and fibronectin binding to integrin alpha 4 beta 1: implications for integrin function and rational drug design. Ciba Found Symp 189, 177-191; discussion 191-179.

147. Brown, P. J., and Juliano, R. L. (1988) Monoclonal antibodies to distinctive epitopes on the alpha and beta subunits of the fibronectin receptor. Exp Cell Res 177, 303-318.

148. Hadjiargyrou, M., Kaprielian, Z., Kato, N., and Patterson, P. H. (1996) Association of the tetraspan protein CD9 with integrins on the surface of S-16 Schwann cells. J Neurochem 67, 2505-2513.

149. Levy, S., and Shoham, T. (2005) Protein-protein interactions in the tetraspanin web. Physiology (Bethesda) 20, 218-224.

150. Horvath, G., Serru, V., Clay, D., Billard, M., Boucheix, C., and Rubinstein, E. (1998) CD19 is linked to the integrin-associated tetraspans CD9, CD81, and CD82. J Biol Chem 273, 30537-30543.

151. Nishi, E., and Klagsbrun, M. (2004) Heparin-binding epidermal growth factorlike growth factor (HB-EGF) is a mediator of multiple physiological and pathological pathways. Growth Factors 22, 253-260.

152. Naglich, J. G., Metherall, J. E., Russell, D. W., and Eidels, L. (1992) Expression cloning of a diphtheria toxin receptor: identity with a heparin-binding EGF-like growth factor precursor. Cell 69, 1051-1061.

153. Nakamura, K., Mitamura, T., Takahashi, T., Kobayashi, T., and Mekada, E. (2000) Importance of the major extracellular domain of CD9 and the epidermal growth factor (EGF)-like domain of heparin-binding EGF-like growth factor for up-regulation of binding and activity. J Biol Chem 275, 18284-18290.

154. Gangarosa, L. M., Dempsey, P. J., Damstrup, L., Barnard, J. A., and Coffey, R. J. (1996) Transforming growth factor-alpha. Baillieres Clin Gastroenterol 10, 4963.

155. Bosenberg, M. W., and Massague, J. (1993) Juxtacrine cell signaling molecules. Curr Opin Cell Biol 5, 832-838.

156. Bringman, T. S., Lindquist, P. B., and Derynck, R. (1987) Different transforming growth factor-alpha species are derived from a glycosylated and palmitoylated transmembrane precursor. Cell 48, 429-440.

157. Shi, W., Fan, H., Shum, L., and Derynck, R. (2000) The tetraspanin CD9 associates with transmembrane TGF-alpha and regulates TGF-alpha-induced EGF receptor activation and cell proliferation. J Cell Biol 148, 591-602.

158. Freed, J. H., and Rosloniec, E. F., Jr. (1988) Molecular aspects of the role of class II MHC molecules in antigen presentation to T cells. Year Immunol 3, 161-178.

159. Villadangos, J. A., Schnorrer, P., and Wilson, N. S. (2005) Control of MHC class II antigen presentation in dendritic cells: a balance between creative and destructive forces. Immunol Rev 207, 191-205.

160. Unternaehrer, J. J., Chow, A., Pypaert, M., Inaba, K., and Mellman, I. (2007) The tetraspanin CD9 mediates lateral association of MHC class II molecules on the dendritic cell surface. Proc Natl Acad Sci U S A 104, 234-239. 
161. Kaji, K., Takeshita, S., Miyake, K., Takai, T., and Kudo, A. (2001) Functional association of CD9 with the Fc gamma receptors in macrophages. J Immunol 166, 3256-3265.

162. Moseley, G. W. (2005) Tetraspanin-Fc receptor interactions. Platelets 16, 3-12.

163. Wierzbicka-Patynowski, I., and Schwarzbauer, J. E. (2003) The ins and outs of fibronectin matrix assembly. J Cell Sci 116, 3269-3276.

164. Wilson, S. H., Ljubimov, A. V., Morla, A. O., Caballero, S., Shaw, L. C., Spoerri, P. E., Tarnuzzer, R. W., and Grant, M. B. (2003) Fibronectin fragments promote human retinal endothelial cell adhesion and proliferation and ERK activation through alpha5beta1 integrin and PI 3-kinase. Invest Ophthalmol Vis Sci 44, 1704-1715.

165. Skorstengaard, K., Jensen, M. S., Petersen, T. E., and Magnusson, S. (1986) Purification and complete primary structures of the heparin-, cell-, and DNAbinding domains of bovine plasma fibronectin. Eur J Biochem 154, 15-29.

166. Kornblihtt, A. R., Umezawa, K., Vibe-Pedersen, K., and Baralle, F. E. (1985) Primary structure of human fibronectin: differential splicing may generate at least 10 polypeptides from a single gene. Embo J 4, 1755-1759.

167. Potts, J. R., and Campbell, I. D. (1996) Structure and function of fibronectin modules. Matrix Biol 15, 313-320; discussion 321.

168. Baron, M., Main, A. L., Driscoll, P. C., Mardon, H. J., Boyd, J., and Campbell, I. D. (1992) 1H NMR assignment and secondary structure of the cell adhesion type III module of fibronectin. Biochemistry 31, 2068-2073.

169. Hynes, R. O. (1992) Integrins: versatility, modulation, and signaling in cell adhesion. Cell 69, 11-25.

170. Chandrasekhar, S., Sorrentino, J. A., and Millis, A. J. (1983) Interaction of fibronectin with collagen: age-specific defect in the biological activity of human fibroblast fibronectin. Proc Natl Acad Sci U S A 80, 4747-4751.

171. Yamada, K. M., Kennedy, D. W., Kimata, K., and Pratt, R. M. (1980) Characterization of fibronectin interactions with glycosaminoglycans and identification of active proteolytic fragments. $J$ Biol Chem 255, 6055-6063.

172. Makogonenko, E., Tsurupa, G., Ingham, K., and Medved, L. (2002) Interaction of fibrin(ogen) with fibronectin: further characterization and localization of the fibronectin-binding site. Biochemistry 41, 7907-7913.

173. Cook, G. A., Wilkinson, D. A., Crossno, J. T., Jr., Raghow, R., and Jennings, L. K. (1999) The tetraspanin CD9 influences the adhesion, spreading, and pericellular fibronectin matrix assembly of Chinese hamster ovary cells on human plasma fibronectin. Exp Cell Res 251, 356-371.

174. Beauchemin, N., Draber, P., Dveksler, G., Gold, P., Gray-Owen, S., Grunert, F., Hammarstrom, S., Holmes, K. V., Karlsson, A., Kuroki, M., Lin, S. H., Lucka, L., Najjar, S. M., Neumaier, M., Obrink, B., Shively, J. E., Skubitz, K. M., Stanners, C. P., Thomas, P., Thompson, J. A., Virji, M., von Kleist, S., Wagener, C., Watt, S., and Zimmermann, W. (1999) Redefined nomenclature for members of the carcinoembryonic antigen family. Exp Cell Res 252, 243-249.

175. Snyder, S. K., Wessner, D. H., Wessells, J. L., Waterhouse, R. M., Wahl, L. M., Zimmermann, W., and Dveksler, G. S. (2001) Pregnancy-specific glycoproteins 
function as immunomodulators by inducing secretion of IL-10, IL-6 and TGFbeta1 by human monocytes. Am J Reprod Immunol 45, 205-216.

176. Waterhouse, R., Ha, C., and Dveksler, G. S. (2002) Murine CD9 is the receptor for pregnancy-specific glycoprotein 17. J Exp Med 195, 277-282.

177. Ellerman, D. A., Ha, C., Primakoff, P., Myles, D. G., and Dveksler, G. S. (2003) Direct binding of the ligand PSG17 to CD9 requires a CD9 site essential for sperm-egg fusion. Mol Biol Cell 14, 5098-5103.

178. Ha, C. T., Waterhouse, R., Wessells, J., Wu, J. A., and Dveksler, G. S. (2005) Binding of pregnancy-specific glycoprotein 17 to $\mathrm{CD} 9$ on macrophages induces secretion of IL-10, IL-6, PGE2, and TGF-beta1. J Leukoc Biol 77, 948-957.

179. Weber, R. L., and Iacono, V. J. (1997) The cytokines: a review of interleukins. Periodontal Clin Investig 19, 17-22.

180. Cruikshank, W. W., Kornfeld, H., and Center, D. M. (2000) Interleukin-16. J Leukoc Biol 67, 757-766.

181. Okubo, Y., Tsukadaira, A., Takashi, S., Kubo, K., and Koyama, S. (2001) Chemotaxis of human CD4+ eosinophils. Int Arch Allergy Immunol 125 Suppl 1, 19-21.

182. Qi, J. C., Wang, J., Mandadi, S., Tanaka, K., Roufogalis, B. D., Madigan, M. C., Lai, K., Yan, F., Chong, B. H., Stevens, R. L., and Krilis, S. A. (2006) Human and mouse mast cells use the tetraspanin CD9 as an alternate interleukin-16 receptor. Blood 107, 135-142.

183. Moissoglu, K., and Schwartz, M. A. (2006) Integrin signalling in directed cell migration. Biol Cell 98, 547-555.

184. Hato, T., Sumida, M., Yasukawa, M., Watanabe, A., Okuda, H., and Kobayashi, Y. (1990) Induction of platelet Ca2+ influx and mobilization by a monoclonal antibody to CD9 antigen. Blood 75, 1087-1091.

185. Kroll, M. H., Mendelsohn, M. E., Miller, J. L., Ballen, K. K., Hrbolich, J. K., and Schafer, A. I. (1992) Monoclonal antibody AG-1 initiates platelet activation by a pathway dependent on glycoprotein IIb-IIIa and extracellular calcium. Biochim Biophys Acta 1137, 248-256.

186. Ozaki, Y., Satoh, K., Kuroda, K., Qi, R., Yatomi, Y., Yanagi, S., Sada, K., Yamamura, H., Yanabu, M., Nomura, S., and et al. (1995) Anti-CD9 monoclonal antibody activates p72syk in human platelets. J Biol Chem 270, 15119-15124.

187. Berditchevski, F., and Odintsova, E. (1999) Characterization of integrintetraspanin adhesion complexes: role of tetraspanins in integrin signaling. J Cell Biol 146, 477-492.

188. Murayama, Y., Miyagawa, J., Oritani, K., Yoshida, H., Yamamoto, K., Kishida, O., Miyazaki, T., Tsutsui, S., Kiyohara, T., Miyazaki, Y., Higashiyama, S., Matsuzawa, Y., and Shinomura, Y. (2004) CD9-mediated activation of the p46 Shc isoform leads to apoptosis in cancer cells. J Cell Sci 117, 3379-3388.

189. Ravichandran, K. S. (2001) Signaling via Shc family adapter proteins. Oncogene 20, 6322-6330.

190. Hong, I. K., Kim, Y. M., Jeoung, D. I., Kim, K. C., and Lee, H. (2005) Tetraspanin CD9 induces MMP-2 expression by activating p38 MAPK, JNK and c-Jun pathways in human melanoma cells. Exp Mol Med 37, 230-239. 
191. Clevers, H. (2006) Wnt/beta-catenin signaling in development and disease. Cell 127, 469-480.

192. Huang, C. L., Liu, D., Masuya, D., Kameyama, K., Nakashima, T., Yokomise, H., Ueno, M., and Miyake, M. (2004) MRP-1/CD9 gene transduction downregulates Wnt signal pathways. Oncogene 23, 7475-7483.

193. Huang, C. L., Ueno, M., Liu, D., Masuya, D., Nakano, J., Yokomise, H., Nakagawa, T., and Miyake, M. (2006) MRP-1/CD9 gene transduction regulates the actin cytoskeleton through the downregulation of WAVE2. Oncogene 25, 6480-6488.

194. Guan, J. L. (1997) Focal adhesion kinase in integrin signaling. Matrix Biol 16, 195-200.

195. Puck, T. T., Cieciura, S. J., and Robinson, A. (1958) Genetics of somatic mammalian cells. III. Long-term cultivation of euploid cells from human and animal subjects. J Exp Med 108, 945-956.

196. Burger, S. R., Remaley, A. T., Danley, J. M., Moore, J., Muschel, R. J., Wunner, W. H., and Spitalnik, S. L. (1991) Stable expression of rabies virus glycoprotein in Chinese hamster ovary cells. J Gen Virol 72 (Pt 2), 359-367.

197. Chen, D., Van Horn, D. J., White, M. F., and Backer, J. M. (1995) Insulin receptor substrate 1 rescues insulin action in $\mathrm{CHO}$ cells expressing mutant insulin receptors that lack a juxtamembrane NPXY motif. Mol Cell Biol 15, 4711-4717.

198. Li, R., Mitra, N., Gratkowski, H., Vilaire, G., Litvinov, R., Nagasami, C., Weisel, J. W., Lear, J. D., DeGrado, W. F., and Bennett, J. S. (2003) Activation of integrin alphaIIbbeta3 by modulation of transmembrane helix associations. Science 300, 795-798.

199. Pijuan, V., Zhuang, Y., Smith, L., Kroupis, C., Condrescu, M., Aceto, J. F., Reeves, J. P., and Smith, J. B. (1993) Stable expression of the cardiac sodiumcalcium exchanger in CHO cells. Am J Physiol 264, C1066-1074.

200. Cook, G. A., Longhurst, C. M., Grgurevich, S., Cholera, S., Crossno, J. T., Jr., and Jennings, L. K. (2002) Identification of CD9 extracellular domains important in regulation of $\mathrm{CHO}$ cell adhesion to fibronectin and fibronectin pericellular matrix assembly. Blood 100, 4502-4511.

201. Brown, P. J., and Juliano, R. L. (1985) Selective inhibition of fibronectinmediated cell adhesion by monoclonal antibodies to a cell-surface glycoprotein. Science 228, 1448-1451.

202. Ng, T., Shima, D., Squire, A., Bastiaens, P. I., Gschmeissner, S., Humphries, M. J., and Parker, P. J. (1999) PKCalpha regulates beta1 integrin-dependent cell motility through association and control of integrin traffic. Embo J 18, 3909-3923.

203. Juliano, R. L., Reddig, P., Alahari, S., Edin, M., Howe, A., and Aplin, A. (2004) Integrin regulation of cell signalling and motility. Biochem Soc Trans 32, 443446.

204. King, W. G., Mattaliano, M. D., Chan, T. O., Tsichlis, P. N., and Brugge, J. S. (1997) Phosphatidylinositol 3-kinase is required for integrin-stimulated AKT and Raf-1/mitogen-activated protein kinase pathway activation. Mol Cell Biol 17, 4406-4418.

205. O'Neill, G. M., Fashena, S. J., and Golemis, E. A. (2000) Integrin signalling: a new Cas $(\mathrm{t})$ of characters enters the stage. Trends Cell Biol 10, 111-119. 
206. Nishiuchi, R., Sanzen, N., Nada, S., Sumida, Y., Wada, Y., Okada, M., Takagi, J., Hasegawa, H., and Sekiguchi, K. (2005) Potentiation of the ligand-binding activity of integrin alpha3beta1 via association with tetraspanin CD151. Proc Natl Acad Sci U S A 102, 1939-1944.

207. Bennett, J. S. (2005) Structure and function of the platelet integrin alphallbbeta3. J Clin Invest 115, 3363-3369.

208. Van de Walle, G. R., Vanhoorelbeke, K., Majer, Z., Illyes, E., Baert, J., Pareyn, I., and Deckmyn, H. (2005) Two functional active conformations of the integrin \{alpha\} 2 \{beta 1 , depending on activation condition and cell type. J Biol Chem 280, 36873-36882.

209. Clark, K., Pankov, R., Travis, M. A., Askari, J. A., Mould, A. P., Craig, S. E., Newham, P., Yamada, K. M., and Humphries, M. J. (2005) A specific alpha5beta1-integrin conformation promotes directional integrin translocation and fibronectin matrix formation. J Cell Sci 118, 291-300.

210. Shimaoka, M., Takagi, J., and Springer, T. A. (2002) Conformational regulation of integrin structure and function. Annu Rev Biophys Biomol Struct 31, 485-516.

211. Luque, A., Sanchez-Madrid, F., and Cabanas, C. (1994) Functional regulation of the human integrin VLA-1 (CD49a/CD29) by divalent cations and stimulatory beta 1 antibodies. FEBS Lett 346, 278-284.

212. Mould, A. P., Barton, S. J., Askari, J. A., Craig, S. E., and Humphries, M. J. (2003) Role of ADMIDAS cation-binding site in ligand recognition by integrin alpha 5 beta $1 . J$ Biol Chem 278, 51622-51629.

213. Ni, H., Li, A., Simonsen, N., and Wilkins, J. A. (1998) Integrin activation by dithiothreitol or $\mathrm{Mn} 2+$ induces a ligand-occupied conformation and exposure of a novel NH2-terminal regulatory site on the beta1 integrin chain. J Biol Chem 273, 7981-7987.

214. Coller, B. S., Peerschke, E. I., Scudder, L. E., and Sullivan, C. A. (1983) A murine monoclonal antibody that completely blocks the binding of fibrinogen to platelets produces a thrombasthenic-like state in normal platelets and binds to glycoproteins IIb and/or IIIa. J Clin Invest 72, 325-338.

215. Kouns, W. C., Wall, C. D., White, M. M., Fox, C. F., and Jennings, L. K. (1990) A conformation-dependent epitope of human platelet glycoprotein IIIa. J Biol Chem 265, 20594-20601.

216. Lu, Y., Carraher, J., Zhang, Y., Armstrong, J., Lerner, J., Rogers, W. P., and Steiner, M. S. (1999) Delivery of adenoviral vectors to the prostate for gene therapy. Cancer Gene Ther 6, 64-72.

217. Ozes, O. N., Mayo, L. D., Gustin, J. A., Pfeffer, S. R., Pfeffer, L. M., and Donner, D. B. (1999) NF-kappaB activation by tumour necrosis factor requires the Akt serine-threonine kinase. Nature 401, 82-85.

218. Locascio, A., and Nieto, M. A. (2001) Cell movements during vertebrate development: integrated tissue behaviour versus individual cell migration. Curr Opin Genet Dev 11, 464-469.

219. Luster, A. D., Alon, R., and von Andrian, U. H. (2005) Immune cell migration in inflammation: present and future therapeutic targets. Nat Immunol 6, 1182-1190.

220. Yamada, K. M. (2000) Fibronectin peptides in cell migration and wound repair. $J$ Clin Invest 105, 1507-1509. 
221. Yamaguchi, H., Wyckoff, J., and Condeelis, J. (2005) Cell migration in tumors. Curr Opin Cell Biol 17, 559-564.

222. Firtel, R. A., and Chung, C. Y. (2000) The molecular genetics of chemotaxis: sensing and responding to chemoattractant gradients. Bioessays 22, 603-615.

223. Even-Ram, S., and Yamada, K. M. (2005) Cell migration in 3D matrix. Curr Opin Cell Biol 17, 524-532.

224. Serrador, J. M., Nieto, M., and Sanchez-Madrid, F. (1999) Cytoskeletal rearrangement during migration and activation of T lymphocytes. Trends Cell Biol 9, 228-233.

225. Higashiyama, M., Taki, T., Ieki, Y., Adachi, M., Huang, C. L., Koh, T., Kodama, K., Doi, O., and Miyake, M. (1995) Reduced motility related protein-1 (MRP$1 / \mathrm{CD} 9)$ gene expression as a factor of poor prognosis in non-small cell lung cancer. Cancer Res 55, 6040-6044.

226. Houle, C. D., Ding, X. Y., Foley, J. F., Afshari, C. A., Barrett, J. C., and Davis, B. J. (2002) Loss of expression and altered localization of KAI1 and CD9 protein are associated with epithelial ovarian cancer progression. Gynecol Oncol 86, 69-78.

227. Miyake, M., Inufusa, H., Adachi, M., Ishida, H., Hashida, H., Tokuhara, T., and Kakehi, Y. (2000) Suppression of pulmonary metastasis using adenovirally motility related protein-1 (MRP-1/CD9) gene delivery. Oncogene 19, 5221-5226.

228. Seymour, L., Bezwoda, W. R., and Meyer, K. (1990) Tumor factors predicting for prognosis in metastatic breast cancer. The presence of $\mathrm{P} 24$ predicts for response to treatment and duration of survival. Cancer 66, 2390-2394.

229. Murillo, C. A., Rychahou, P. G., and Evers, B. M. (2004) Inhibition of alpha5 integrin decreases PI3K activation and cell adhesion of human colon cancers. Surgery 136, 143-149.

230. Jaken, S. (1996) Protein kinase C isozymes and substrates. Curr Opin Cell Biol 8, 168-173.

231. Mellor, H., and Parker, P. J. (1998) The extended protein kinase C superfamily. Biochem J 332 ( Pt 2), 281-292.

232. Webb, B. L., Hirst, S. J., and Giembycz, M. A. (2000) Protein kinase C isoenzymes: a review of their structure, regulation and role in regulating airways smooth muscle tone and mitogenesis. Br J Pharmacol 130, 1433-1452.

233. Kawakami, T., Kawakami, Y., and Kitaura, J. (2002) Protein kinase C beta (PKC beta): normal functions and diseases. J Biochem (Tokyo) 132, 677-682.

234. Nakashima, S. (2002) Protein kinase C alpha (PKC alpha): regulation and biological function. J Biochem (Tokyo) 132, 669-675.

235. Saito, N., and Shirai, Y. (2002) Protein kinase C gamma (PKC gamma): function of neuron specific isotype. J Biochem (Tokyo) 132, 683-687.

236. Cybulsky, A. V., Carbonetto, S., Cyr, M. D., McTavish, A. J., and Huang, Q. (1993) Extracellular matrix-stimulated phospholipase activation is mediated by beta 1-integrin. Am J Physiol 264, C323-332.

237. Haller, H., Lindschau, C., Maasch, C., Olthoff, H., Kurscheid, D., and Luft, F. C. (1998) Integrin-induced protein kinase Calpha and Cepsilon translocation to focal adhesions mediates vascular smooth muscle cell spreading. Circ Res 82, 157-165.

238. Pacifici, R., Roman, J., Kimble, R., Civitelli, R., Brownfield, C. M., and Bizzarri, C. (1994) Ligand binding to monocyte alpha 5 beta 1 integrin activates the alpha 
2 beta 1 receptor via the alpha 5 subunit cytoplasmic domain and protein kinase C. J Immunol 153, 2222-2233.

239. Martiny-Baron, G., Kazanietz, M. G., Mischak, H., Blumberg, P. M., Kochs, G., Hug, H., Marme, D., and Schachtele, C. (1993) Selective inhibition of protein kinase C isozymes by the indolocarbazole Go 6976. J Biol Chem 268, 9194-9197.

240. Zhu, X., and Assoian, R. K. (1995) Integrin-dependent activation of MAP kinase: a link to shape-dependent cell proliferation. Mol Biol Cell 6, 273-282.

241. Schlessinger, J. (2000) Cell signaling by receptor tyrosine kinases. Cell 103, 211225.

242. Juliano, R. L. (2002) Signal transduction by cell adhesion receptors and the cytoskeleton: functions of integrins, cadherins, selectins, and immunoglobulinsuperfamily members. Annu Rev Pharmacol Toxicol 42, 283-323.

243. Renshaw, M. W., Ren, X. D., and Schwartz, M. A. (1997) Growth factor activation of MAP kinase requires cell adhesion. Embo J 16, 5592-5599.

244. Mitra, S. K., Mikolon, D., Molina, J. E., Hsia, D. A., Hanson, D. A., Chi, A., Lim, S. T., Bernard-Trifilo, J. A., Ilic, D., Stupack, D. G., Cheresh, D. A., and Schlaepfer, D. D. (2006) Intrinsic FAK activity and Y925 phosphorylation facilitate an angiogenic switch in tumors. Oncogene 25, 5969-5984.

245. Lin, T. H., Chen, Q., Howe, A., and Juliano, R. L. (1997) Cell anchorage permits efficient signal transduction between ras and its downstream kinases. J Biol Chem 272, 8849-8852.

246. Slack-Davis, J. K., Eblen, S. T., Zecevic, M., Boerner, S. A., Tarcsafalvi, A., Diaz, H. B., Marshall, M. S., Weber, M. J., Parsons, J. T., and Catling, A. D. (2003) PAK1 phosphorylation of MEK1 regulates fibronectin-stimulated MAPK activation. J Cell Biol 162, 281-291.

247. Alessi, D. R., Cuenda, A., Cohen, P., Dudley, D. T., and Saltiel, A. R. (1995) PD 098059 is a specific inhibitor of the activation of mitogen-activated protein kinase kinase in vitro and in vivo. J Biol Chem 270, 27489-27494.

248. Dudley, D. T., Pang, L., Decker, S. J., Bridges, A. J., and Saltiel, A. R. (1995) A synthetic inhibitor of the mitogen-activated protein kinase cascade. Proc Natl Acad Sci U S A 92, 7686-7689.

249. Vanhaesebroeck, B., Leevers, S. J., Ahmadi, K., Timms, J., Katso, R., Driscoll, P. C., Woscholski, R., Parker, P. J., and Waterfield, M. D. (2001) Synthesis and function of 3-phosphorylated inositol lipids. Annu Rev Biochem 70, 535-602.

250. Rameh, L. E., and Cantley, L. C. (1999) The role of phosphoinositide 3-kinase lipid products in cell function. J Biol Chem 274, 8347-8350.

251. Nolte, R. T., Eck, M. J., Schlessinger, J., Shoelson, S. E., and Harrison, S. C. (1996) Crystal structure of the PI 3-kinase p85 amino-terminal SH2 domain and its phosphopeptide complexes. Nat Struct Biol 3, 364-374.

252. Reiske, H. R., Kao, S. C., Cary, L. A., Guan, J. L., Lai, J. F., and Chen, H. C. (1999) Requirement of phosphatidylinositol 3-kinase in focal adhesion kinasepromoted cell migration. J Biol Chem 274, 12361-12366.

253. Okada, T., Kawano, Y., Sakakibara, T., Hazeki, O., and Ui, M. (1994) Essential role of phosphatidylinositol 3-kinase in insulin-induced glucose transport and antilipolysis in rat adipocytes. Studies with a selective inhibitor wortmannin. $J$ Biol Chem 269, 3568-3573. 
254. Nakanishi, S., Kakita, S., Takahashi, I., Kawahara, K., Tsukuda, E., Sano, T.,

Yamada, K., Yoshida, M., Kase, H., Matsuda, Y., and et al. (1992) Wortmannin, a microbial product inhibitor of myosin light chain kinase. J Biol Chem 267, 2157 2163.

255. Rao, K., Paik, W. Y., Zheng, L., Jobin, R. M., Tomic, M., Jiang, H., Nakanishi, S., and Stojilkovic, S. S. (1997) Wortmannin-sensitive and -insensitive steps in calcium-controlled exocytosis in pituitary gonadotrophs: evidence that myosin light chain kinase mediates calcium-dependent and wortmannin-sensitive gonadotropin secretion. Endocrinology 138, 1440-1449.

256. Vlahos, C. J., Matter, W. F., Hui, K. Y., and Brown, R. F. (1994) A specific inhibitor of phosphatidylinositol 3-kinase, 2-(4-morpholinyl)-8-phenyl-4H-1benzopyran-4-one (LY294002). J Biol Chem 269, 5241-5248.

257. Stoll, V., Calleja, V., Vassaux, G., Downward, J., and Lemoine, N. R. (2005) Dominant negative inhibitors of signalling through the phosphoinositol 3-kinase pathway for gene therapy of pancreatic cancer. Gut 54, 109-116.

258. Bai, H., Pollman, M. J., Inishi, Y., and Gibbons, G. H. (1999) Regulation of vascular smooth muscle cell apoptosis. Modulation of bad by a phosphatidylinositol 3-kinase-dependent pathway. Circ Res 85, 229-237.

259. Hara, K., Yonezawa, K., Sakaue, H., Ando, A., Kotani, K., Kitamura, T., Kitamura, Y., Ueda, H., Stephens, L., Jackson, T. R., and et al. (1994) 1Phosphatidylinositol 3-kinase activity is required for insulin-stimulated glucose transport but not for RAS activation in CHO cells. Proc Natl Acad Sci U S A 91, 7415-7419.

260. Song, G., Ouyang, G., and Bao, S. (2005) The activation of Akt/PKB signaling pathway and cell survival. J Cell Mol Med 9, 59-71.

261. Datta, S. R., Brunet, A., and Greenberg, M. E. (1999) Cellular survival: a play in three Akts. Genes Dev 13, 2905-2927.

262. Meier, R., and Hemmings, B. A. (1999) Regulation of protein kinase B. J Recept Signal Transduct Res 19, 121-128.

263. Panetti, T. S. (2002) Tyrosine phosphorylation of paxillin, FAK, and p130CAS: effects on cell spreading and migration. Front Biosci 7, d143-150.

264. Defilippi, P., Di Stefano, P., and Cabodi, S. (2006) p130Cas: a versatile scaffold in signaling networks. Trends Cell Biol 16, 257-263.

265. Parsons, J. T., Martin, K. H., Slack, J. K., Taylor, J. M., and Weed, S. A. (2000) Focal adhesion kinase: a regulator of focal adhesion dynamics and cell movement. Oncogene 19, 5606-5613.

266. Shi, Q., and Boettiger, D. (2003) A novel mode for integrin-mediated signaling: tethering is required for phosphorylation of FAK Y397. Mol Biol Cell 14, 43064315.

267. Klemke, R. L., Leng, J., Molander, R., Brooks, P. C., Vuori, K., and Cheresh, D. A. (1998) CAS/Crk coupling serves as a "molecular switch" for induction of cell migration. J Cell Biol 140, 961-972.

268. Stipp, C. S., Kolesnikova, T. V., and Hemler, M. E. (2003) Functional domains in tetraspanin proteins. Trends Biochem Sci 28, 106-112. 
269. Argraves, W. S., Suzuki, S., Arai, H., Thompson, K., Pierschbacher, M. D., and Ruoslahti, E. (1987) Amino acid sequence of the human fibronectin receptor. $J$ Cell Biol 105, 1183-1190.

270. Bellis, S. L., Newman, E., and Friedman, E. A. (1999) Steps in integrin beta1chain glycosylation mediated by TGFbeta 1 signaling through Ras. J Cell Physiol 181, 33-44.

271. Pickering, J. G., Chow, L. H., Li, S., Rogers, K. A., Rocnik, E. F., Zhong, R., and Chan, B. M. (2000) alpha5betal integrin expression and luminal edge fibronectin matrix assembly by smooth muscle cells after arterial injury. Am J Pathol 156, 453-465.

272. Wilkins, J. A., Li, A., Ni, H., Stupack, D. G., and Shen, C. (1996) Control of beta1 integrin function. Localization of stimulatory epitopes. J Biol Chem 271, 3046-3051.

273. Velling, T., Nilsson, S., Stefansson, A., and Johansson, S. (2004) beta1-Integrins induce phosphorylation of Akt on serine 473 independently of focal adhesion kinase and Src family kinases. EMBO Rep 5, 901-905.

274. Armulik, A., Velling, T., and Johansson, S. (2004) The integrin beta1 subunit transmembrane domain regulates phosphatidylinositol 3-kinase-dependent tyrosine phosphorylation of Crk-associated substrate. Mol Biol Cell 15, 25582567.

275. Lewis, J. M., and Schwartz, M. A. (1995) Mapping in vivo associations of cytoplasmic proteins with integrin beta 1 cytoplasmic domain mutants. Mol Biol Cell 6, 151-160.

276. Lemmon, M. A., Ferguson, K. M., and Abrams, C. S. (2002) Pleckstrin homology domains and the cytoskeleton. FEBS Lett 513, 71-76.

277. Innocenti, M., Frittoli, E., Ponzanelli, I., Falck, J. R., Brachmann, S. M., Di Fiore, P. P., and Scita, G. (2003) Phosphoinositide 3-kinase activates Rac by entering in a complex with Eps8, Abi1, and Sos-1. J Cell Biol 160, 17-23.

278. Price, L. S., Leng, J., Schwartz, M. A., and Bokoch, G. M. (1998) Activation of Rac and Cdc42 by integrins mediates cell spreading. Mol Biol Cell 9, 1863-1871.

279. Nobes, C. D., and Hall, A. (1995) Rho, rac, and cdc42 GTPases regulate the assembly of multimolecular focal complexes associated with actin stress fibers, lamellipodia, and filopodia. Cell 81, 53-62.

280. Higuchi, M., Masuyama, N., Fukui, Y., Suzuki, A., and Gotoh, Y. (2001) Akt mediates Rac/Cdc42-regulated cell motility in growth factor-stimulated cells and in invasive PTEN knockout cells. Curr Biol 11, 1958-1962. 


\section{VITA}

Jayaprakash Kotha, was born on October 27, 1973 in Machilipatnam, India. He graduated from the College of Medicine at the Guntur Medical College, Guntur, India with a Bachelor of Medicine and Bachelor of Surgery (M.B.B.S) in the year 1997. In the fall of 2001, he joined the graduate school at The University of Tennessee Health Science Center, Memphis, TN. 